\title{
LA DEDICA DEGLI ATENIESI PER LA VITTORIA SU BEOTI E CALCIDESI DEL 506 A.C. (IG I³ 501) E LA SUA COLLOCAZIONE TOPOGRAFICA
}

\author{
STEFANO BERTI \\ Memoria presentata dal m.e. Cinzia Bearzot \\ (Adunanza del 14 aprile 2011)
}

\begin{abstract}
The monument - a quadriga - the Athenians dedicated on the Acropolis for their victory over the Boeotians and the Chalcidians in 506 B.C. has always aroused great interest, both in antiquity and down to the present day: not only is the monument well attested by ancient sources, but it was also restored around the middle of the $5^{\text {th }}$ century, as witnessed by inscriptions; consequently, the $6^{\text {th }}$ century original and the $5^{\text {th }}$ century restoration have been the subject of a long scholarly debate. This paper focuses on the topographical context of the victory monument and that of its restoration, trying to ascertain where they were dedicated. Accordingly, modern theories are critically reviewed and ancient sources thoroughly examined. The latter, together with epigraphical, religious and political reasons, suggest that the original quadriga and its restoration were erected on the same site inside the Acropolis.
\end{abstract}

Nel Supplemento all'Enciclopedia dell'Arte Antica si legge: “alla prima affermazione militare della nuova democrazia si deve il primo grande anathema bronzeo dell'Acropoli, quella quadriga dei Beoti e Calcidesi (506 a.C.) la cui dislocazione dall'area occidentale del tempio pisistrateo allo spazio antistante al propileo arcaico e poi nuovamente contro l'analemma occidentale del tempio arcaico sembra appurata". ${ }^{1}$ Come si può notare dalle parole utilizzate ("sembra appurata"), ci troviamo al termine di un aspro dibattito topografico, caratterizzato da numerose proposte, complesse e contrastanti, sino a prevedere lo spostamento della quadriga,

RINGRAZIAMENTO: Desidero ringraziare la prof. Cinzia Bearzot dell'Università Cattolica di Milano per il continuo sostegno e la pazienza dimostrata; ringrazio anche la dott. Sara Chiarini (Freie Universität Berlin) per alcuni consigli in ambito archeologico e letterario. La responsabilità di quanto espongo, errori compresi, rimane mia.

1 L. BESCHI, Atene, in Enciclopedia dell'arte antica, Secondo Suppl. 1971-1994, I, Roma 1994, 496-518: 503. 
soluzione oggi - a quanto pare - preferita. Il monumento in questione è la dedica che gli Ateniesi innalzarono per celebrare la vittoria del 506 a.C. sui Beoti e sui Calcidesi, abbandonati dagli Spartani nel corso del progettato attacco alla giovane democrazia. Il ritiro degli Spartani è spiegato da Erodoto con l'opposizione corinzia al tentativo, da parte di Cleomene, di insediare con la forza Isagora tiranno di Atene: il dissenso sarebbe poi stato raccolto dall'altro re spartano, Demarato. ${ }^{2}$ Sono state trovate altre motivazioni, che ho già avuto modo di sintetizzare. ${ }^{3}$ Qui vorrei invece approfondire la collocazione topografica del famoso anathema.

\section{LE ORIGINI DEL PROBLEMA}

Molte sono le fonti che ci hanno tramandato la vittoria ateniese del 506 a.C., ${ }^{4}$ ma soltanto due sono state utilizzate per ricavare la posizione della quadriga celebrativa: Erodoto (V 77, 3-4) e Pausania (I 28, 2).

2 Her. V 75, 1. Cfr. P. CARLIER, Cleomene I, re di Sparta, in Contro le "leggi immutabili". Gli Spartani fra tradizione e innovazione, a c. di C. BEARZOT - F. LANDUCCI, Milano 2004 (Contributi di storia antica, 2), 33-51: 45-46.

3 S. BERTI, La dedica degli Ateniesi per la vittoria su Beoti e Calcidesi del 506 a.C. (IG I3 501) e la data del suo ripristino, «Aevum», 84 (2010), 7-40: 16-17; ulteriori proposte, tra cui la (solo tentata?) alleanza fra Atene e la Persia sono discusse p. es. in K.W. WelweI, Das klassische Athen, Darmstadt 1999, 24-25 con note e bibliografia; U. BULTRIGHINI, Cleomene, Erodoto e gli altri, in Storiografia e regalità nel mondo greco. Colloquio interdisciplinare. Cattedre di Storia della Storiografia Greca e Storia Greca. Chieti, 17-18 gennaio 2002, a c. di E. LUPPINO MANES, Alessandria 2003, 51-119: 95 n. 130, con bibliografia (coll'avvertenza però che la definizione dell'intervento di Cleomene come "non statale" non deve essere attribuita all'invasione del 506 a.C., ma a quella precedente, cfr. Her. V 74-75 e M. MEIER, Aristokraten und Damoden. Untersuchungen zur inneren Entwicklung Spartas im 7. Jabrbundert v. Chr. und zur politischen Funktion der Dichtung des Tyrtaios, Stuttgart 1998, 115-116). V. ora S. BERTI, The Athenian Victory over the Boeotians and the Chalcidians (506 B.C.) in the Light of the Epigraphical Findings, «AHB», 24 (2010), 3-23 con discussione della spiegazione di L.A. TRITLE, Kleomenes at Eleusis, «Historia», 37 (1988), 457-60.

4 Her. V 74-78; Diod. X 24, 3; P.Oxy. 2535; Paus. I 28, 2; Ael. Arist. XIII 120 pp. 194-95 DindorF = I 91 pp. 39-40 LENZ-BEHR; Him. II 12 DUEBNER = VI 12 Colonna; forse Ael. Varia Hist VI 1. Erodoto, Diodoro e l'Antologia Palatina (VI 343) citano per intero l'epigramma per la vittoria, mentre P.Oxy. 2535 ed Ael. Arist. XLIX 380 p. 512 DINDORF = XXVIII 64 p. 162 KEIL riportano soltanto il primo verso e metà del secondo. Su queste fonti cf. BERTI, La dedica degli Ateniesi, 7-8. 
Erodoto, in particolare, ricorda anche dove furono collocati i ceppi dei Beoti e dei Calcidesi fatti prigionieri, ad ulteriore memoria del successo di Atene, come del resto sottolineato nella base iscritta della

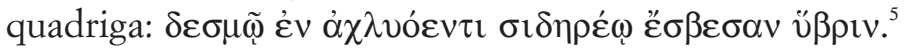

HER. V $77,3-4^{6}$

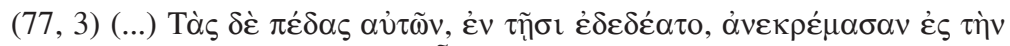

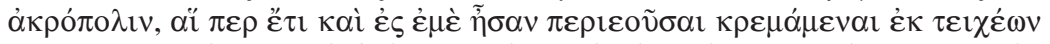

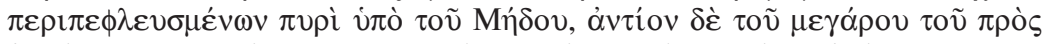

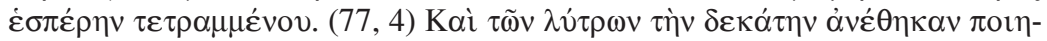

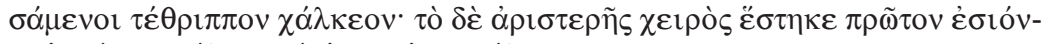

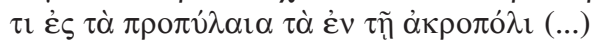

$(77,3)$ (...) Ma i loro ceppi, in cui erano stati legati, li appesero sull'Acropoli, dove erano rimasti fino al mio tempo, appesi alle mura bruciacchiate dal fuoco acceso dal Medo, davanti al megaron volto ad Occidente. $(77,4)$ E dedicarono la decima dei riscatti, avendo fabbricato una quadriga di bronzo; e questa per prima si erge alla mano sinistra per chi entra nei propilei dell'Acropoli (...)

Pausania invece, nel corso della sua descrizione dell'Acropoli, semplicemente accomuna nel ricordo l'Atena Promachos e la quadriga, in quanto entrambe erette con la decima di un bottino di guerra.

\section{PAUS. I $28,2^{7}$}

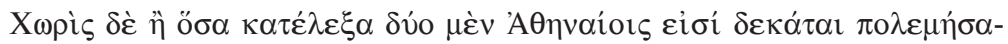

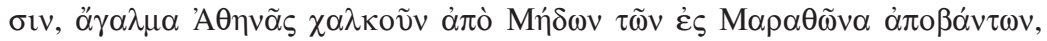

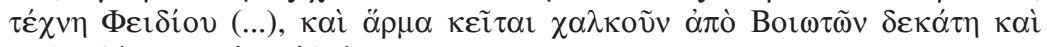

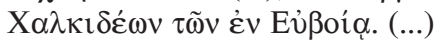

Oltre ai monumenti che ho già passato in rassegna, gli Ateniesi ne hanno due come decime del bottino di guerre da loro sostenute: una

5 Her. V 77, 4.

6 Testo: Herodoti Historiae, II, ed. H.B. Rosén, Stutgardiae et Lipsiae 1997. Traduzione: Erodoto. Le Storie, V, a c. di G. NENCI, Milano 1994, con adattamenti, in parte ispirati a Le Storie di Erodoto, II, a c. di A. ColonnA - F. BEVILACQUA, Torino 1996.

Testo: Pausaniae Graeciae Descriptio, I, rec. Fr. SPIRO, Lipsiae 1903. Traduzione: Pausania. Guida della Grecia, I, intr., testo e trad. a c. di D. Musti, comm. a c. di L. BeSCHI - D. Musti, Milano 1982, con adattamenti. 
statua in bronzo di Atena, col bottino preso ai Medi sbarcati a Maratona, opera di Fidia (...); c'è poi un carro di bronzo, decima proveniente da una vittoria sui Beoti e sui Calcidesi di Eubea. (...)

Leggendo Erodoto, quindi, sembra chiaro che la quadriga si trovasse a sinistra per chi entrava nei Propilei; il problema sorge dal confronto con Pausania: poiché ha appena nominato la statua di Atena Promachos e sta seguendo un itinerario topograficamente abbastanza preciso ${ }^{8}$ si è soliti considerare inevitabile la vicinanza ad essa della quadriga, in corrispondenza di un taglio nella roccia che in pratica verrebbe però a trovarsi $a$ destra per chi entrava nei Propilei.

Si è quindi cercato di privilegiare una fonte rispetto all'altra, oppure di conciliarle. Naturalmente il problema si intreccia con il noto rifacimento cui andò incontro l'anathema, distrutto dai Persiani nel 480 e ripristinato, come ho cercato di dimostrare, poco dopo il $457 .{ }^{10}$ Peraltro, qualunque data si voglia assegnare al monumento rinnovato, è evidente che Erodoto si riferisce a quest'ultimo, in quanto ne registra la particolare versione dell'iscrizione dedicatoria. Possediamo infatti sia un frammento dell'iscrizione di fine VI secolo sia i frammenti di quella di metà del V, che riportano l'epigramma con i medesimi versi ma in ordine differente:

$I G \mathrm{I}^{3} 501^{11}$

\section{A}





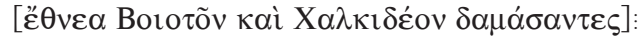

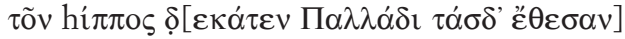

B

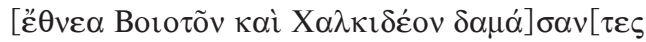

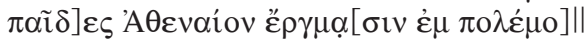

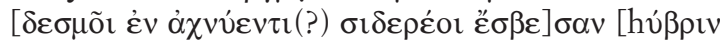

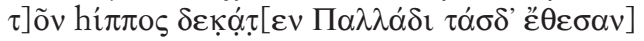

8 L. Beschi - D. Musti, in Pausania. Guida della Grecia, I, intr., testo e trad. a c. di Musti, comm. a c. di BesCHI - Musti, XCIII-CI.

9 V. infra.

10 BERTI, La dedica degli Ateniesi, 19-40.

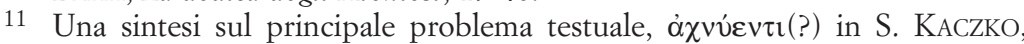
From Stone to Parchment: Epigraphic and Literary Transmission of Some Greek Epigrams, «TiC», 1 (2009), 90-117: 113 n. 69; cfr. BERTI, La dedica degli Ateniesi, 9-11. 
A

In una ? catena di ferro spensero la tracotanza

i figli degli Ateniesi nelle imprese di guerrall

avendo domato i popoli dei Beoti e dei Calcidesi;

come decima da essi dedicarono a Pallade queste cavalle

B

Avendo i figli degli Ateniesi domato i popoli

dei Beoti e dei Calcidesi nelle imprese di guerra,\|

in una ? catena di ferro ne spensero la tracotanza;

come decima da essi dedicarono a Pallade queste cavalle

Per alcuni l'inversione nella coppia di distici ha assunto valore anche topografico ed è quindi parte del dibattito in corso. ${ }^{12}$ Per il momento si può comunque ricordare che fu ormai la nuova dedica ad essere vista da entrambe le nostre fonti.

C'è poi una terza fonte, purtroppo frammentaria: il lacerto di commentario papiraceo P.Oxy 2535 riporta infatti, dopo una parziale citazione del nostro epigramma celebrativo riconosciuta da E. Lobel e E.G. Turner, ${ }^{13}$ una serie di osservazioni che ne illustrano il contesto storico ma anche, per l'appunto, topografico:

P.OXY $2535^{14}$

]$\delta .[] \pi \mathrm{\imath}$

$\delta .[.().] \pi \mathrm{\imath}$

]. $\mu \omega v \mathrm{r}$

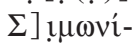

]. $\varepsilon \rho \iota \kappa о v$

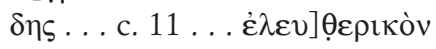

12 v. par. 3.2.1 e 3.2.17.

13 E.G. TURNER, Simonides and the Epigram on the Fetters of the Chalcidians in a New Papyrus, «PCA», 59 (1962), 21-22; ID., in The Oxyrbynchus Papyri, XXXI, ed. by J.W.B. BARnS - P. PARSONS - J. REA - E.G. TuRner, London 1966, 14-16. Il papiro viene datato palograficamente al tardo I secolo d.C.

14 Testo: a sinistra la proposta di ricostruzione di TURNER (LOBEL) in The Oxyrbynchus Papyri, XXXI, ed. by BARNS - PARSONS - REA - TURNER, 15; a destra la proposta di ricostruzione di D. SIDER, Simonides Epigram 3 FGE in P.Oxy. 31.2535, «ZPE», 162 (2007), 5-8: 6, con adattamenti (nella trascrizione Sider conservava un unico sigma lunato alla r. 4, di conseguenza qui uniformato alle convenzioni della sua trascrizione; alla r. 12 Sider scriveva per una svista $\tau \varepsilon \tau \varepsilon \tilde{i} \sigma \theta \alpha \mathrm{l}$ in luogo del normale $\tau \varepsilon \theta \varepsilon \tilde{\imath} \sigma \theta \alpha \mathrm{l}$ leggibile sul papiro e qui riportato, come già da Turner; alla r. 15 il termine Eúßot $\alpha v$ è naturalmente proparossitono; alla r. 16 si è deciso di inserire direttamente nel testo la congettura $\dot{\varepsilon} \kappa \tau \tilde{\eta} \varsigma$ 'A $\tau \iota \kappa \tilde{n} \varsigma$ formulata da A. PETROVIC per e-mail a Sider e da quest'ultimo registrata con approvazione in D. SIDER, recensione a L. BRAVI, Gli epigrammi di Simonide e le vie della tradizione, Roma 2006 (Filologia e critica 94), «BMCRev», 2008.02.47 n. 7.) 


\begin{tabular}{|c|c|}
\hline 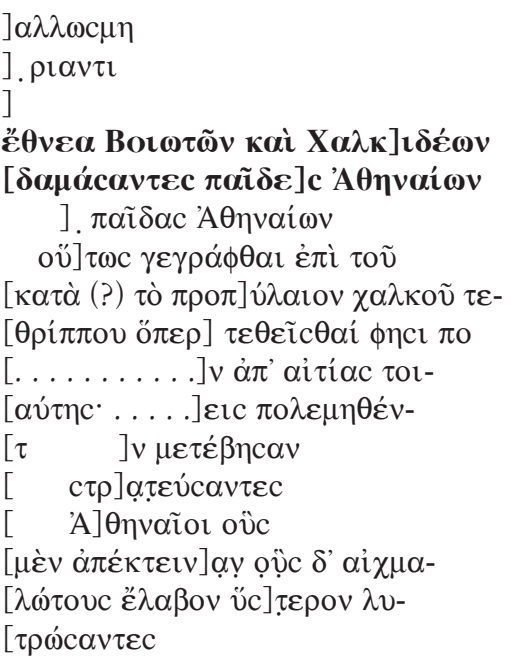 & 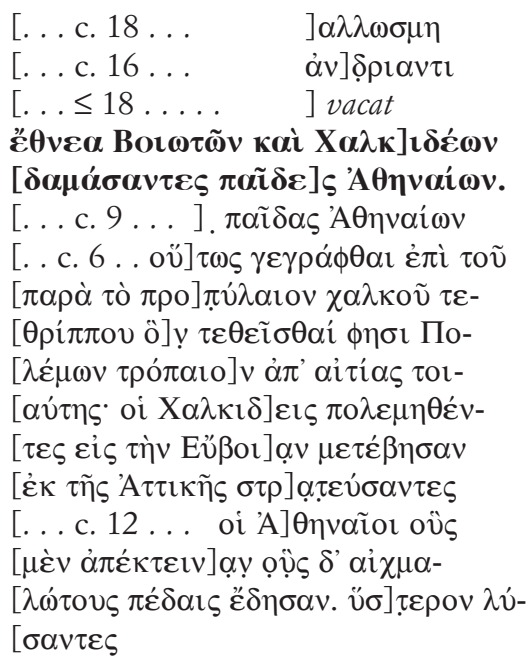 \\
\hline
\end{tabular}

Tra le osservazioni, dunque, spicca la menzione, ricostruibile con sicurezza, del Propylon: quest'ultimo doveva con ogni probabilità essere considerato, come i Propylaia di Erodoto, un punto di riferimento, non lontano dal quale doveva trovarsi la quadriga. Le integrazioni pro-

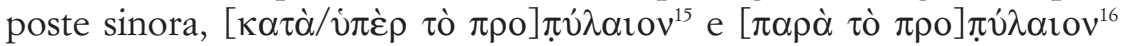
sembrerebbero andare in questa direzione, ma la frammentarietà del testo e la genericità del riferimento non permettono di trarre indicazioni più specifiche, anche se la fonte del commentario potesse essere identificata in un'osservazione autorevole ed eventualmente anche indipendente da Erodoto quale quella di un Polemone di Ilio, ${ }^{17}$ come suggerito con prudenza da Turner ${ }^{18}$ e ribadito con maggiore decisione da Sider: ${ }^{19}$ il $\phi \eta \sigma r$ di riga 12, infatti, sembrerebbe preludere alla citazione

15 Lobel ap. Turner, in The Oxyrhynchus Papyri, XXXI, ed. by BARns PARSONS - REA - TURNER, 15.

16 D. SIDER, Simonides Epigram 3 FGE, 6-7.

17 Secondo la Suda (IV p. 158 ADLER, s.v. Пo $\lambda \dot{\mu} \mu \omega v$ n $^{\circ} 1888$ ) sarebbe vissuto al tempo di Tolemeo Epifane (204-180 a.C.). Per l'autorevolezza di Polemone cfr. K. DeICHgräBer, Polemon $\mathrm{n}^{\circ}$ 9, in RE, XXI (1952), 1288-1320 e A.A. Donohue, Polemon $\mathrm{n}^{\circ}$ 2, DNP, X (2001), 7.

18 TuRner, in The Oxyrbynchus Papyri, XXXI, ed. by BARNS - PARSONS - REA TURNER, 15-16.

19 SIDER, Simonides Epigram 3 FGE, 7-8. 
dell'autore che è alla base delle notizie riportate nel commentario; se il

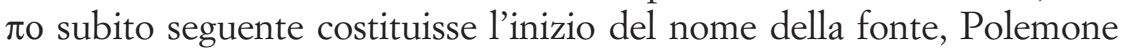

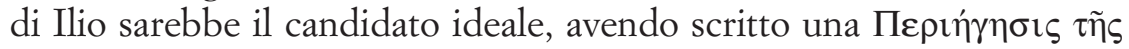

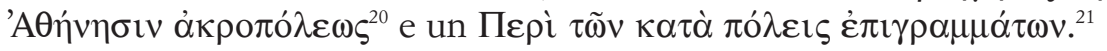

\section{I RESTI MATERIALI}

I frammenti della base iscritta sono le uniche parti dei due successivi monumenti che siano effettivamente giunte sino a noi.

La base della fine del VI secolo era composta da due pietre, di cui la prima è perduta, mentre della seconda è conservato al Museo Epigrafico di Atene un frammento (EM 6286) mutilo sulla destra e sul retro (dimensioni: h. $0,30 \mathrm{~m}, 1.0,51 \mathrm{~m}, \mathrm{sp} .0,37 \mathrm{~m}$ ), ma con anathyrosis sulla faccia sinistra. ${ }^{22}$ Il materiale prescelto è la pietra scura di Eleusi, l'iscrizione è in alfabeto attico arcaico (altezza delle lettere: v. 1 0,025-0,03 m; v. 2 0,022-0,025 m, entrambi compresi fra due linee guida). Fu ritrovata sull'Acropoli, a Nord-Est dei Propilei, in particolare, secondo la dettagliata descrizione di H.G. Lolling, fra le rovine del grosso edificio proprio a Nord-Est dei Propilei, a Est della grossa cisterna ora interrata, e precisamente nella sezione meridionale dell'edificio, una stoa, solo pochi passi dal portico orientale dei Propilei. ${ }^{23}$

La base del V secolo era anch'essa formata da due pietre, delle quali sono conservati al Museo Epigrafico di Atene quattro frammenti

20 FHG III 1-5 cfr. 6, pp. 116-17.

21 FHG III 79-80, pp. 138-39.

22 Ricostruzione: A.E. RAUBITSCHEK, Dedications from the Athenian Akropolis, Cambridge MA 1949, 193. Foto: RAUBITSCHEK, Dedications, 192; L. JEFFERY, The Local Scripts of Archaic Greece, Oxford 1961, tav. 4 n 43; B. VIRGILIO, Commento storico al quinto libro delle "Storie" di Erodoto, Pisa 1975 (Biblioteca degli studi classici e orientali, 4), tav. VII.

23 H.G. Lolling in A. KiRCHHOFf (H.G. LOLLING), Bemerkungen zu dem Bruchstück einer Basis von der Burg zu Athen, «SPAW», (1887), 111-14: 112 (= H.G. LOLLING in IG, I suppl. ed. A. KIRCHHOFF, Berolini 1877-1886-1891, 78 n 334 a): "In den Ruinen des grossen Gebäudes gleich nord-östlich von den Propyläen, östlich von der jetzt zugeschütteten grossen Cisterne gefunden, und zwar in der südlichen Atheilung des Baues, einer Stoa, nur wenige Schritte von der Osthalle der Propyläen.” 
mutili da entrambe le parti, integri solo superiormente, uno appartenente alla prima pietra (fr. a: EM 6287 $\alpha$ ), tre (fr. b: EM 12410; fr. c: senza indicazione; fr. d: EM 6287) - fra loro congiungentisi - alla seconda (dimensioni: fr. a h. $0.11 \mathrm{~m}, 1.0,12 \mathrm{~m}, \mathrm{sp} .0,14 \mathrm{~m}$; fr. b, c, d uniti fra loro h. $0,18 \mathrm{~m}, 1.0,665 \mathrm{~m}, \mathrm{sp} .0,43 \mathrm{~m}) \cdot{ }^{24} \mathrm{Il}$ materiale del rifacimento è il "marmo pentelico", l'iscrizione, stoichedica, è in alfabeto attico, con sigma a tre tratti (altezza delle lettere: 0,025 m). Purtroppo il luogo di rinvenimento è in questo caso fornito genericamente: ''Acropoli. $^{25}$

La base sosteneva, secondo l'unanime testimonianza di Erodoto (V 77, 4) e Pausania (I 28, 2), una quadriga di bronzo, ma non sono stati trovati buchi per perni o tasselli sulla parte superiore dei frammenti pervenutici: ciò si può spiegare, secondo A.E. Raubitschek, con il confronto con $I G \mathrm{I}^{3} 880$, dove i primi buchi distano $41 \mathrm{~cm}$ dal bordo anteriore della base. ${ }^{26}$ Sempre un confronto con IG I 752 e IG I3 880 rende probabile che la base in esame, anzi le basi (l'originale e il frutto del restauro), fossero costituite da due pietre di uguale lunghezza accostate fra loro. ${ }^{27}$ L'integrazione del testo fa pensare che le basi fossero altresì di uguali dimensioni ${ }^{28}$ con una lunghezza dell'iscrizione di circa 2,85 $\mathrm{m}$ e di conseguenza una larghezza della base di almeno 3 metri. ${ }^{29}$

Sul materiale impiegato per la costruzione della quadriga abbia-

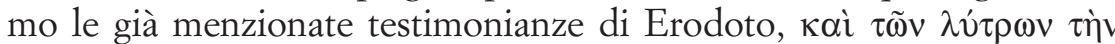

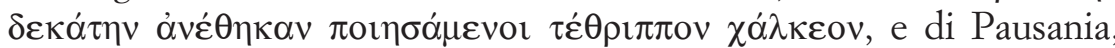

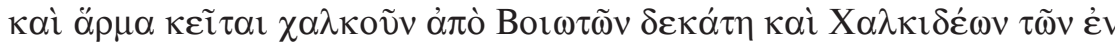
Ev̉ßoía: dunque il bronzo. Sappiamo però che Erodoto e Pausania videro soltanto la copia di V secolo del monumento originale di VI: di che materiale era quest'ultimo?

24 Ricostruzione: RaubitscheK, Dedications, 202. Foto: RaubitscheK, Dedications, 202; VIRGILIO, Commento storico, tav. VIII; G. NENCI, Erodoto, V, fig. 30

25 A. KIRCHHOFF, Über zwei attische Votivinschriften aus Perikleischer Zeit, in 27. Mai. Gesammtsitzung der Akademie, «Monatsber. Akad. Berlin», (1869), 409-16: 409.

26 Raubitschek, Dedications, 205.

27 M. JACOB-FELSCH, Die Entwicklung griechischer Statuenbasen und die Aufstellung der Statuen, Waldsassen 1969, 31 considera quanto rimane dell'originale il primo esempio conservato di base con questa tecnica.

28 RAuBITSCHEK, Dedications, 205.

29 Così sin da C.H. Weller, The Pre-Periclean Propylon, «AJA», 8 (1904), $35-$ 70: 64-65 n. 1; L. WEBER, гrKA 'EФ EPMHI IV, «Philologus», 77 (1921), 77-108: 98; W. JudEICH, Topographie von Athen, München 1931², 239. 
Raubitschek ritiene probabile che il monumento originario, come quello che lo sostituì, fosse di bronzo, ma non escluderebbe neppure il marmo: $:^{30}$ è stato suggerito, infatti, che due protomi equine di marmo appartenessero al monumento.

L'ipotesi, per la verità avanzata solamente exempli gratia, era di G. Dickins, ${ }^{31}$ ripresa con molta cautela da W.H. Schuchhardt. ${ }^{32}$ Si sarebbe trattato delle due protomi equine, di grandezza all'incirca naturale (altezza di quanto è rimasto della prima: $1,19 \mathrm{~m}$; altezza di quanto è rimasto della seconda: $0,73 \mathrm{~m}$ ), una meglio conservata, ${ }^{33}$ che si trovava nel cortile dell'allora Museo dell'Acropoli, di fronte all'entrata, e una conservata meno bene, ${ }^{34}$ che si trovava nel deposito del medesimo Museo, già sulla scalinata dei Propilei. Ora sono entrambe esposte all'interno del Nuovo Museo dell'Acropoli, con numero di inventario, rispettivamente, Akr. 6454 e Akr. 15244. Il loro impiego in un frontone al modo dei cavalli del Tempio di Apollo a Delfi ${ }^{35}$ era da Schuchhardt considerato improbabile a causa della superficie posteriore completamente liscia, priva quindi, a differenza dei cavalli di Delfi, di segni di un'opportuna congiunzione a mortasa. ${ }^{36}$ Per motivi stilistici Schuchhardt datava le protomi intorno al 520 a.C. (comunque non più antiche del cosiddetto "cavaliere persiano"), senza però escludere una datazione più bassa: in questo caso e ipotizzando una quadriga anziché una biga si sarebbe potuto costruire un rapporto con la dedica per la vittoria del $506 .{ }^{37}$ Raubitschek segue da

30 RAUBITSCHEK, Dedications, 193-94.

31 G. Dickins, Catalogue of the Acropolis Museum, I, Cambridge 1912, 56.

32 W.H. SCHUCHHARDT, Rundwerke außer den Koren. Reliefs, $n^{\circ} 321$, in Die archaische Marmorbildwerke der Akropolis, hrsg. von H. SCHRADER, bearb. von E. LANGLOTZ - W.H. SCHUCHHARDT - H. SCHRADER, Frankfurt a. M. 1939, 242-44.

33 SchuchHardt, Rundwerke, fig. 271, cfr. H. PAYNe - G. MACKWORTHYoung, Archaic marble Sculpture from the Acropolis, London $1950^{2}$, tav. $135 \mathrm{n}^{\circ} 2$. Ora v. anche M.B. Moore, The Central Group in the Gigantomachy of the Old Athena Temple on the Acropolis, «AJA», 99 (1995), 633-39: 634-35 fig. 1-3.

34 SChuchHARDT, Rundwerke, fig. 272-73. Ora v. anche Moore, The Central Group, 636-37 fig. 4-5.

35 Disegno ricostruttivo dei frontoni del Tempio di Delfi: P. DE LA CosTEMesselière, Fouilles de Delphes, IV, 3, Sculptures des Temples, Paris 1931, fig. 7-8; resti dei cavalli: ID., Fouilles, IV, 3, 23-24 (frontone ovest) e 40-43 (frontone est).

36 SCHUCHHARDT, Rundwerke, 244.

37 SCHUCHHARDT, Rundwerke, 242-44, con descrizione analitica delle protomi e valutazione della loro qualità artistica; cfr. G. DiCKINS, Catalogue, 55-56. 
vicino Schuchhardt, scrivendo che tali statue parrebbero appartenere a una quadriga o a una biga, piuttosto che ad una scultura frontonale, rappresentando la parte frontale di ciascun cavallo, sino all'addome, con il corpo poi troncato uniformemente, liscio, e collegato al plinto da un supporto di marmo: la collocazione dinanzi a un muro, infatti, come previsto dal taglio nella roccia assegnato alla dedica per la vittoria sui Beoti e sui Calcidesi da G.Ph. Stevens, ${ }^{38}$ si armonizzerebbe con una rappresentazione della sola metà anteriore della quadriga; anche le dimensioni si accorderebbero con quelle della base iscritta e, di nuovo, con quelle del taglio nella roccia. ${ }^{39}$ Sulla stessa linea anche $I G \mathrm{I}^{3} 501$ : "de donario prisco utrum aenei equi essent an marmorei (cf. Schuchhardt, Rundwerke $\mathrm{n}$. 321) incertum manet". " In generale osservava Chr.W. Clairmont ${ }^{41}$ che allo stato attuale delle nostre conoscenze della scultura di bronzo e di marmo di fine VI secolo un gruppo marmoreo sarebbe stato a priori più probabile.

Tuttavia K. Stähler riteneva di aver dimostrato mediante un'analisi stilistica ed il confronto proprio con il Tempio di Apollo a Delfi l'opportunità di una quadriga al centro del frontone occidentale del Tempio arcaico di Atena ${ }^{42}$ e vi ha quindi ricondotto quelle protomi equine, anche grazie alla somiglianza del materiale utilizzato: un marmo a grana grossa con striature blu, come nel caso dell'Atena e del Gigante del medesimo frontone. ${ }^{43}$ Siamo ormai sicuri, del resto, che le protomi rappresentassero in origine una quadriga, grazie allo studio, da parte di M.B. Moore, dei segni lasciati sul marmo dal sistema di bardature usuale per quattro cavalli. ${ }^{44}$ In seguito anche M. Korres, nel ribadire la tesi

38 G.Ph. Stevens, The Periclean Entrance Court, «Hesperia», 5 (1936), 443 520: 492 fig. $42 \mathrm{n}^{\circ} 2$.

39 RAUBITSCHEK, Dedications, 193-94.

$40 I G, I .2^{3}$, ed. D. LEWIS - L.H. JefFERY adiuv. E. ERXLEBEN, Berlin-New York $1994,490 n^{\circ} 501$

41 Chr.W. Clairmont, Patrios Nomos, I, Oxford 1983, 92.

42 K. STÄHLER, Zur Rekonstruktion und Datierung des Gigantomachiegiebels von der Akropolis, in Antike und Universalgeschichte. Festschrift H. E. Stier zum 70. Geburtstag am 25. Mai 1972, hrsg. von R. STIEHE - G. A. LEHMANN, Münster 1972, 88 112: 96-98, con ulteriori paralleli a p. 99 n. 39.

43 K. STÄHLER, Zur Rekonstruktion, 98-101 + tav. 1.

44 MoOre, The Central Group, 633-39; cfr. anche G. Dickins, Catalogue, 56 e F. WINTER, Archaische Reiterbilder von der Akropolis, «JDAI», 8 (1893), 135-56: 138. 
di Stähler, l'ha considerata definitivamente confermata pure dai recenti ritrovamenti del timpano, ${ }^{45}$ sebbene vadano segnalati i dubbi in merito alla ricostruzione avanzati, ad esempio, da J.R. Marszal, che propone tra l'altro di attribuire la quadriga e Atena al frontone opposto. ${ }^{46} \mathrm{Al}$ contrario, ragioni di spazio, stilistiche, e soprattutto il luogo di ritrovamento dei cavalli - le pendici settentrionali dell'Acropoli a differenza di tutti gli altri elementi frontonali - accuratamente indagato nella storia degli scavi e degli studi dell'Acropoli, hanno portato F. Santi ad escludere del tutto l'appartenenza dei cavalli ad uno dei frontoni del tempio, senza per questo dare credito all'ipotesi di una loro esposizione come anathema, ipotesi invece recuperata da G.I. Despinis, che ne ha prospettato il reimpiego dedicatorio, insieme con il torso del Museo Nazionale Inv. 3045, in luogo dell'originaria, ma alla fine non realizzata, destinazione frontonale: per entrambi gli studiosi, quindi, i cavalli non sarebbero mai stati collocati su alcun frontone; le valutazioni divergono sulla datazione, subito dopo la fine della tirannide per Despinis, mentre per Santi sarebbero comunque più recenti della dedica per la vittoria sui Beoti e sui Calcidesi. ${ }^{47}$

A proposito di quest'ultima si è invece solitamente concordi nell'osservare che la scelta del tutto inconsueta di una quadriga per celebrare una vittoria in guerra sarebbe stata favorita dal fatto che proprio la quadriga era una specialità militare di Beoti e Calcidesi. ${ }^{48}$ Tuttavia P.

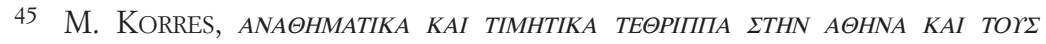
$\Delta E \Lambda \Phi O r \Sigma$, in Delphes cent ans aprés la Grande Fouille. Essai du bilan. Actes du Colloque International, Athènes-Delphes 17-20 septembre 1992, ed. par A. JACQUEMIN, Paris 2000 (BCH Suppl. 36), 293-329: 312-14.

46 J.R MARSZAL, An Epiphany for Athena: the Eastern Pediment of the Old Athena Temple at Athens, in $\Sigma T E \Phi A N O \Sigma$ : Studies in Honor of Brunilde Sismondo Ridgway, ed. by K.J. HARTSWicK - M. StURGEON, Philadelphia 1998, 173-80. Medesimi dubbi, con accenno all'eventualità che la quadriga potesse appartenere al frontone orientale già in A. DELIVORRIAS, Attische Giebelskulpturen und Akrotere des fünften Jabrbunderts, Tübingen 1974 (Tübinger Studien zur Archäologie und Kunstgeschichte, 1), 179.

47 F. SANTI, I cavalli inv. 6454 e 15244 del Museo dell'Acropoli ed il frontone della Gigantomachia, «ArchClass», 55 (2004), 1-23; G.I DesPINIS, Der Torso Athener Nationalmuseum Inv. 3045. Fragment einer Giebelkomposition mit Athenageburt?, «AA», 2009, 1-10: 5-7.

48 L.H. JEFFERY, Greece before the Persian Invasion, in The Cambridge Ancient History, IV, ed. by J. BOARDMAN - N.G.L. HAMmond - D.M. LEWIS - M. OsTWALD, Cambridge 19882, 347-67: 362. E. REISCH, Griechische Weibgeschenke, Wien 1890 
Schollmeyer ha fatto notare che il combattimento con i carri non poteva più essere in uso a quest'epoca: ${ }^{49}$ le testimonianze si concentrano infatti in età omerica ${ }^{50}$ mentre in epoca storica questa consuetudine bellica andava scemando, mantenendosi viva solamente in aree periferiche, ${ }^{51}$ anche se vi è chi non escluderebbe un uso geograficamente più diffuso, seppure in maniera sporadica, ancora nel VI secolo, in particolare in Beozia. ${ }^{52}$ Certo però il carro ed i cavalli costituivano dei simboli per Calcide, se si pensa alle prime monete conosciute: ${ }^{53}$ è stata anche tracciata un'evoluzione dalle prime caratteristiche rappresentazioni di quadrighe viste frontalmente a più sintetiche ruote, intendendole come abbreviazioni per la quadriga ${ }^{54}$ è aperta la questione se una serie di monete beotiche con la ruota sul verso possano avere un'origine analoga, oppure si tratti di simboli solari, o ancora si tratti di consapevole imitazione di modelli calcidesi: ${ }^{55}$ è con un discreto grado di certezza, invece, che si

(Abhandlungen des Archäologisch-Epigraphischen Seminares der Universität Wien, 8), 148 e W.H.D. Rouse, Greek Votive Offerings. An Essay in the History of Greek Religion, Cambridge 1902, 114-15 parlavano di vera e propria allusione alle spoglie della battaglia. 49 P. SchollmeYer, Antike Gespanndenkmäler, Hamburg 2001 (Antiquitates 13), 59.

50 H. van WeEs, Greek Warfare. Myths and Realities, London 2004, 158-60; completamente scettico è P.A.L GreENHALGH, Early Greek Warfare. Horsemen and Chariots in the Homeric and Archaic Ages, Cambridge 1973, 18, secondo il quale i carri che compaiono sul campo di battaglia nell'epica o nell'arte geometrica sarebbero nient'altro che "racing chariots transferred to a military use by deliberately archaicizing Dark Age bards and by similarly archaicizing Geometric artists influenced by the epic"; cfr. anche A. SNODGRASS, Early Greek Armour and Weapons from the End of the Bronze Age to 600 B.C., Edinburgh 1964, 159-63.

51 Come a Salamina di Cipro o a Cirene, cfr. F. CHAmoux, La civilisation grecque a l'époque archaique et classique, Paris 1963, 152.

52 VAN WEES, Greek Warfare, 176-77; con cautela aggiunge infatti a Cirene e a Salamina di Cipro Eretria e la Beozia M. DetiEnne, Remarques sur le char en Grèce, in Problèmes de la guerre en Grèce, dir. de J.P. VERNANT, Paris-La Haye 1968, 313-18: 318; Tessaglia e Beozia avrebbero mantenuto l'uso del carro da guerra per U. WILAMOWITZ, Euripides. Herakles, II, Berlin 1895, 114, seguito da L. LACROIX, Le bouclier, emblème des Béotiens, «RBPh», 36 (1958), 5-30: 10 + tav. I, che al carro da guerra associa anche una terracotta beotica del VI secolo.

53 F. JÜNGER, Gespann und Herrschaft. Form und Intention grossformatiger Gespanndenkmäler im griechischen Kulturraum von der archaischen bis in die bellenistische Zeit, Hamburg 2006 (Antiquitates 36), 233-35 sottolinea con forza come l'appropriazione ateniese di un simbolo calcidese serva a marcare la vittoria di Atene e la conseguente occupazione del territorio degli sconfitti.

54 C.M. KraAY, Archaic and Classical Greek Coins, London 1976, 89-90; cfr. G.K. Jenkins, Monnaies grecques, Fribourg 1972, 51-52. 
datano proprio a questo periodo, con allusione all'alleanza tra Beoti e Calcidesi, due esemplari, ${ }^{56}$ conservati rispettivamente a Berlino e a Parigi, di un tetradramma calcidese coniato su piede euboico, che riporta sul recto lo scudo beotico con iscritto il chi $(\Psi)$ calcidese, sul verso la ruota calcidese all'interno di un quadrato incuso. Del resto anche il lessico è indicativo: i Beoti erano detti $\pi \lambda \hat{\eta} \xi \iota \pi \pi \mathrm{or},{ }^{57}$ con resti lessicali in età classi$\mathrm{Ca}^{58}$ mentre i ricchi Calcidesi proprio Erodoto li chiama i $\pi \pi$ oßó $\tau \alpha \mathbf{l}$ (V 77, 2). Il riferimento della nostra quadriga potrebbe quindi essere ai simboli dei popoli vinti più che alle spoglie della battaglia (e anche in questo caso, ovviamente, si sarebbe comunque trattato di una semplice allusione, visto che la quadriga era in bronzo e rappresentava tecnicamente la decima del riscatto, come testimoniato da Erodoto, da Pausania e dal testo dell'epigramma: non una spoglia di guerra). ${ }^{59} \mathrm{Si}$ è pensato poi anche ad una vittoria militare rappresentata attraverso una vittoria atletica ${ }^{60}$ (nella corsa con i carri), continuazione ${ }^{61}$ o superamento ${ }^{62}$ delle dediche aristocratiche. Ipotesi articolate e suggestive sono state espresse da M. Rausch, ${ }^{63}$ il quale,

55 Le diverse posizioni riassunte in KRAAY, Archaic and Classical Greek Coins, 109-110, con bibliografia e A. SCHACHTER, Boiotia in the Sixth Century B.C., in Boiotika. Vorträge vom 5. internationalen Böotien Kolloquium zu Ehren von Professor Dr. Siegfried Lauffer. Institut für Alte Geschichte, Ludwig-Maximilians-Universität München 13.-17. Juni 1986, hrsg. von H. BEISTER - J. BUCKLER, München 1989, 73-86: 85 , con nota 43 e bibliografia.

56 BABELON, Traité, II.1, n 1372 e KRAAY, Archaic and Classical Greek Coins, $\mathrm{n}^{\circ} 266$.

57 Ps. Hes. Scutum 24 = Fragmenta Hesiodea 195 v. 24 MerkelbaCH - WeST; ulteriori epiteti in WILAMOWITZ, Euripides. Herakles, II, 114.

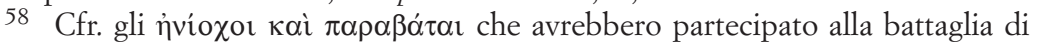
Delio (424 a.C.) secondo Diod. XII 70, 1: si trattava probabilmente di un contingente scelto di opliti (VAN WEES, Greek Warfare, 59).

59 Per un esempio di carro vero e proprio dedicato a tutti gli effetti come spoglia di guerra si può ricorrere allo ל̌ṽyo (così l'iscrizione, ma il termine vale anche per quadrighe e carri in genere, cfr. A Greek-English Lexicon, by H.G. LIDDELL - R. SCOTT, rev. and augm. by H.S. JONES, with R. MCKENZIE, Oxford 19969', 754) sottratto ai Cartaginesi e offerto ad Apollo a Delfi probabilmente da parte di Timoleonte, v. H. POMTOW, Ein sicilisches Anathem in Delphi, «MDAI(A)», 20 (1895), 483-94 con fonti; cfr. L. PRANDI, Le dediche di Timoleonte a Corinto per la vittoria del Crimiso, «RIL», 111 (1977), 35-43: 41 42 n. 25 per un'esauriente discussione della bibliografia; un nuovo tentativo di ricostruzione della dedica è presentato da G. MANGANARO, Epiro "Adriatico" e Sicilia: colonizzazione timoleontea e monete, «Hesperìa», 15 (2002), 113-22: 116-17.

60 C.M. KeEsling, The Votive Statues of the Athenian Acropolis, Cambridge 2003, 175; ID., The Callimachus Monument on the Athenian Acropolis (CEG 256) and Athenian Commemoration of the Athenian Persian Wars, in Archaic and classical Greek 
anche sulla scorta delle coeve rappresentazioni vascolari, ha parlato in primo luogo di un'identificazione con i guerrieri omerici, che ben si armonizzerebbe con la particolare espressione "figli degli Ateniesi" ( $\pi \alpha \tilde{i} \delta \varepsilon \varsigma$ 'A $A \varepsilon v \alpha i \omega v)$ presente nell'epigramma dedicatorio, "un'espressione poetica di ascendenza omerica (cfr. il frequentissimo vĩ $\varepsilon \varsigma$ 'A $\chi \alpha i \tilde{\omega} v)$ che mira a presentare un popolo come antico e nobile" ${ }^{64}$ un richiamo insomma alla tradizione: in questo senso assai opportunamente G. Anderson menziona il Catalogo delle navi, ove (Hom. Iliad. II 551) proprio gli

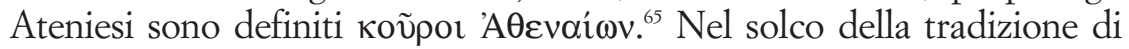
cui Omero è rappresentante, quindi, ma anche, secondo P. Siewert, per sottolineare maggiormente il ruolo della comunità, si spiegherebbe la scelta - qui per la prima volta testimoniata - dell'espressione $\pi \alpha \tilde{i} \delta \varepsilon \varsigma$

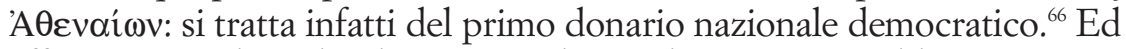
effettivamente la scelta di $\pi \alpha \tilde{i} \delta \varepsilon \varsigma$ in luogo di кoṽpor potrebbe essere una conseguenza della nuova forma di governo democratica: è stata infatti

epigram, ed. by M. Baumbach - A. Petrovic - I. Petrovic, Cambridge 2010, 100-130: 123-24, con nuovi argomenti a sostegno. Chi volesse però ulteriormente osservare l'identità di genere (femminile) delle cavalle di solito celebrate negli epinici con quelle del nostro epigramma ( $\tau$ ó $\sigma \delta^{\prime}$ hí $\left.\pi \pi \mathrm{s}\right)$ si scontrerebbe in realtà con un comune uso poetico, già omerico, (A Greek-English Lexicon, by LIDDELL - SCOTT, rev. and augm. by JONES, with MCKENZIE, 835), che sembra destinare in prevalenza cavalle sia alla corsa sia alla guerra (peraltro ad es. Paus. VI 10, 6-7 riporta un epigramma per la vittoria di tre cavalli maschi ad Olimpia). Prudentemente G. HaFnER, Viergespanne in Vorderansicht. Die repräsentative Darstellung der Quadriga in der griechischen und der späteren Kunst, Berlin 1938 (Neue deutsche Forschungen, Abteilung Archäologie, 2), 55 descriveva la nostra quadriga semplicemente come "ebenfalls votiv für einen Sieg" in rapporto alle più usuali quadrighe celebranti vittorie sportive.

61 SCHOLLMEYER, Antike Gespanndenkmäler, 61.

62 E. KLuwe, Das Siegesmal der Athener für ihren Triumph über die Böoter und Chalkidier im Jahre 506 v. Chr., in Bildergeschichte. Festschrift Klaus Stähler, hrsg. von J. Gebauer - E. Grabow - F. JÜnger - D. Metzler, Mohnesee 2004, 265-76: 274-76.

63 M. RausCH, Isonomia in Athen: Veränderungen des öffentlichen Lebens vom Sturz der Tyrannis bis zur zweiten Perserabwehr, Frankfurt am Main 1999, 124-25.

64 Le Storie di Erodoto, II, a c. di Colonna - BeVIlacQua, 82; cfr. D. Boedeker, Presenting the Past in Fifth Century Athens, in Democracy, Empire and the Arts in Fifth-Century Athens, ed. by D. BOEDEKER - K. A. RAAFLAub, Cambridge, MaLondon 1998 (Center for Hellenic Studies Colloquia, 2), 185-202: 200: “a Homericsounding tag that tends to associate the Athenian hoplites with traditional epic valor".

65 G. Anderson, The Athenian Experiment. Building an Imagined Political Community in Ancient Attica, 508-490 B.C., Ann Arbor 2003, 156.

66 P. SIEWERT, L'autocoscienza politica degli Ateniesi in epoca arcaica e la nascita del monumento nazionale dopo la caduta della tirannide, in Autocoscienza e rappresentazione dei 
osservata una successiva contrapposizione tra un uso normalmente "democratico" dell'espressione $\pi \alpha \tilde{\imath} \delta \varepsilon \varsigma$ 'A $\theta \varepsilon v \alpha i \omega v^{67}$ e la ripresa in con-

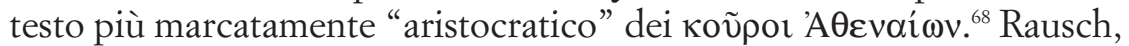
nell'ottica della polisemicità di un'opera d'arte e sempre col supporto di opportuni confronti iconografici, ${ }^{69}$ ha poi istituito l'ulteriore connessione, omerica e mitologica in genere, con il carro da guerra delle divinità, segnatamente di Atena, che è la dedicataria della quadriga ed anche del vicino tempio arcaico: su questo sarà necessario tornare. ${ }^{70}$ Atena stessa, in ogni caso, veniva associata ai cavalli con gli epiteti di I $\pi \pi i \alpha^{71}$ o X $\alpha \lambda \imath v \tilde{\imath} \tau \iota \varsigma^{72}{ }^{72}$ come ha sottolineato A. Petrovic, che ha anche brillantemente individuato la fine ironia sottesa al nostro epigramma: $\mathrm{i}$ Beoti e i Calcidesi, come si è visto noti allevatori e domatori di cavalli, domati dagli Ateniesi ( $\delta \alpha \mu \alpha ́ \sigma \alpha \nu \tau \varepsilon \varsigma)$, che ne celebrano la sconfitta proprio con statue equine (hí $\pi \pi \mathrm{s}) !^{73}$

Infine, sono state proposte allusioni forse troppo sottili al campo di battaglia: a proposito del materiale prescelto per la base originale, la pietra scura di Eleusi, Raubitschek, osservandone l'uso nelle prime dediche sull'Acropoli soltanto qui e in $I G I^{3}$ 866, fa presente che gli Ateniesi, prima di sconfiggere Beoti e Calcidesi, si erano opposti ai Peloponnesiaci proprio nella piana di Eleusi, da cui il materiale proviene ${ }^{74}$ più arrischiata la tesi di R.M. Burrows e P.N. Ure, secondo i quali lo scontro con i Beoti sarebbe potuto avvenire nei pressi di "Ap $\mu \alpha$ in Beozia e la quadriga, essendo un carro (öp $\mu \alpha)$, ricorderebbe il campo di battaglia: ${ }^{75}$ gli autori della proposta sono innanzitutto consapevoli

popoli nell'antichità, a c. di M. SORDI, Milano 1992 (Contributi dell'Istituto di Storia Antica, 18), 3-17: 12.

67 Cfr. gli epigrammi XVIII e XXIV PAGE sulle Guerre Persiane e l'epitafio IG $\mathrm{I}^{3} 1179$ sui caduti a Potidea nel 432 a.C.

68 L. BeRTELLI, L'epigramma per i morti di Tanagra, «QUCC», 6 (1968), 52-98: 62-63 a proposito di $I G \mathrm{I}^{3} 1181$, con confronti letterari.

69 RAUSCH, Isonomia, 124 con nn. 568-72; in generale sull'iconografia di Atena sul carro cfr. P. Demargne, Athena, in LIMC, II.1 (1984), 955-1044: 974 A9.

70 V. par. 4.

71 Paus. I 30, 4; I 31, 6 (cfr. Suid. II p. 659 AdLeR s.v. 'I $\pi \pi \varepsilon i ́ \alpha$ 'A $\theta \eta v \tilde{\alpha}$ n$^{\circ}$ 542).

72 Paus. II 4, 1, per aver imbrigliato Pegaso.

73 A. Petrovic, Kommentar zu den simonideischen Versinschriften, LeidenBoston 2007 (Mnemosyne Suppl. 282), 216.

74 RaubitscheK, Dedications, 193.

75 R.M. BuRROws - P.N. URE, Excavations at Rhitsonna in Boeotia, «ABSA», 14

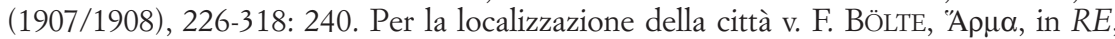
VII (1912), 2366-67. 
che Erodoto parli di $\tau \varepsilon \dot{\theta} \theta \rho \imath \pi$ ov e non di öp $\mu \alpha$, termine che non appare neppure nell'epigramma dedicatorio ma soltanto in Pausania; si tratterebbe comunque di un riferimento ben nascosto, limitato peraltro in questo caso allo scontro con i soli Beoti; si aggiunga poi il carattere di ipotesi costruita su di un'altra ipotesi (relativa allo svolgimento delle operazioni militari) di una simile proposta. ${ }^{76}$

\section{LA COLLOCAZIONE DELLE QUADRIGHE: IL DIBATTITO}

All'inizio fu sicuramente il ritrovamento nel 1869 del primo dei frammenti della base rinnovata ad attirare l'attenzione degli studiosi sulla quadriga e in particolare sulla questione topografica, ma il vero snodo è costituito dal ritrovamento nel 1887 del frammento della base originale, che evidenziò un ordine di versi differente e che fu spiegato da Kirchhoff con una variazione nella posizione tra originale e copia. ${ }^{77}$ Nacque così la teoria che vedeva nello spostamento della quadriga la soluzione al contrasto individuato dagli studiosi fra i due passi menzionati di Erodoto e Pausania. Non per questo la discussione si esaurì. Nel 1936 Stevens, che condivideva la teoria dello spostamento sviluppata in precedenza, affermò di aver individuato dei tagli nella roccia ("rock cuttings") che si sarebbero adattati bene, per dimensioni e contesto archeologico, al basamento della quadriga. ${ }^{78} \mathrm{Nel}$ dibattito, fino allora egualmente incentrato sulle fonti letterarie come sull'archeologia, furono considerate come un dato di fatto ormai acquisito e quindi un punto di partenza le conclusioni archeologiche cui era giunto Stevens, che si era invece servito con pari dignità delle fonti letterarie e dell'archeologia. Di conseguenza, pochi elementi si aggiunsero ad un quadro che non fu più ridiscusso in maniera radicale, né in genere vennero prese in considerazione localizzazioni diverse da quelle ipotizzate da Stevens (che le precisò successivamente), ${ }^{79}$

76 Sullo svolgimento delle operazioni militari dei Beoti, dei Calcidesi e degli Ateniesi alla luce delle testimonianze epigrafiche v. ora BERTI, The Athenian Victory over the Boeotians and the Chalcidians, 3-23.

\footnotetext{
77 V. par. 3.2.1.

78 V. par. 3.2.16

79 V. par. 3.2.16.
} 
spesso neppure da parte di chi non era convinto dalla teoria dello spostamento, divenuta nel frattempo maggioritaria. ${ }^{80}$

Sembra perciò opportuno ripercorrere con una certa ampiezza la storia degli studi riguardanti la quadriga e la sua collocazione, di volta in volta evidenziando e discutendo direttamente le principali tematiche emerse ed i contributi più significativi, tra $i$ quali alcuni, sebbene ormai trascurati, appaiono particolarmente utili e validi ancora oggi. ${ }^{81}$

\subsection{IL DIBATTITO SINO AL 1887}

\subsection{1) PRIMA DEI RITROVAMENTI EPIGRAFICI: LEAKE, BEULÉ}

Una fase preliminare, per così dire "preistorica" (in quanto precedente al ritrovamento dei frammenti iscritti delle basi), può essere a titolo d'esempio rappresentata dalle pubblicazioni di W.M. Leake e di C.E. Beulé. Il primo collocava ancora senza problemi la Promachos e la quadriga tra l'Eretteo e i Propilei sulla scorta di Erodoto e di Pausania; la quadriga, in particolare, veniva a trovarsi a sinistra entrando nell'Acropoli, senza che per questo Pausania ed Erodoto fossero messi in contrapposizione. ${ }^{82}$ Lì si pensava di posizionare semplicemente (verrebbe da dire: "innocentemente") la quadriga prima che i ritrovamenti

80 V. par. 3.2.19-3.2.20.

81 Non si pretende qui di esporre tutta la bibliografia esistente, ma di darne soltanto un'idea: una breve rassegna sino al 1895 in Pausaniae Graeciae Descriptio, ed. H. Hitzig, comm. add. H. HitZIG - H. BlÜMnER, I, Berlin 1896, 303-05; una bibliografia completa al 1949 in RAUBITSCHEK, Dedications, 191-93; 201-02.

82 W.M. LEAKE, The Topography of Athens with Some Remarks on Its Antiquities, London 1821, 244: "He [scil. Pausanias] mentions the statue of Minerva Promachos before the brazen chariot, after which monument he notices only two statues before he concludes his description of the Acropolis, thus agreeing with Herodotus, who places the brazen chariot immediately on the left hand of the entrance"; cfr. $242+$ tav. VI: "as the words of Pausanias indicates that it [scil. the Promachos] stood near the brazen quadriga dedicated from the spoils of Chalcis, which, according to Herodotus, was on the left hand of the traveller as he entered the Acropolis through the Propylaea, it appears evident that the Minerva Promachos must have occupied some part of the space between the Erechtheium and Propylaea, near the Pelasgic, or northern wall"; simili le considerazioni ampliate nella seconda edizione (London 18412 , 351-52; la collocazione della quadriga alla tav. III). 
delle basi dessero impulso agli studi. Anche Beulé, infatti, collocava la quadriga a sinistra dei Propilei, in direzione delle mura settentrionali dell'Acropoli, ${ }^{83}$ seguendo però il solo Erodoto, poiché Pausania “n'indique pas sa situation" ${ }^{84}$ Quest'ultima osservazione anticipava già uno degli esiti possibili dell'ulteriore discussione sviluppatasi all'interno del dibattito topografico riguardante la quadriga: vale a dire se in questo passo (o addirittura in generale) le parole di Pausania potessero sostenere un'interpretazione topografica. ${ }^{85}$ Persino profetico si dimostrava poi Beulé quando scriveva che "il est probable qu'on retrouvera un jour la base du quadrige, en enlevant les masures et les ruines modernes accumulées sur toute cette partie de l'Acropole". ${ }^{86}$

\subsection{2) LA SCOPERTA DEL FRAMMENTO DI ISCRIZIONE D'ETÀ PERICLEA: KIRCHHOFF}

Come è noto, quando il primo frammento venne alla luce Kirchhoff pensò inizialmente che potesse appartenere al monumento originale ${ }^{87}$ datandolo però paleograficamente tra il 452 e il 444 a.C., una datazione naturalmente fin troppo precisa ${ }^{88} \mathrm{ma}$ che si sarebbe rivelata non lontana dal vero. Kirchhoff si rendeva conto di quanto più strano sarebbe stato se la dedica fosse sopravvissuta alle distruzioni persiane, ${ }^{89}$ ma allora scartava la possibilità di essere di fronte ad una copia del-

83 C.E. Beulé, L'acropole d'Athènes, II, Paris 1854, tav. 1 (= Paris $1862^{2}$, tav. 3 ).

84 Beulé, L'acropole, II, Paris 1854, 308 (= Paris 1862², 396).

85 V. par. 3.1.8, 3.1.11, 3.2.1, 3.2.2, 3.2.10, 3.2.20, 4.

86 Beulé, L'acropole, II, Paris 1854, 308 (= Paris $\left.1862^{2}, 396\right)$.

87 A. KIRCHHOFF, Über zwei attische Votivinschriften, 410.

88 Cfr. BERTI, La dedica degli Ateniesi, 12; P.J. RHODES, After the Three-bar Sigma Controversy: the History of Athenian Imperialism Reassessed, «CQ», 58 (2008), 500-06; in generale v. H.B. MatTinglY, The Athenian Empire Restored, Ann Arbor MI 1996.

89 A. KIRCHHOFF, Über zwei attische Votivinschriften, 412. Senza tener conto della scoperta del frammento di V secolo scriveva T.H. DyER, Ancient Athens: Its History, Topography, and Remains, London 1873, 440: "as the victory over the Boeotians and Chalcidians was several years previous to the capture of Athens by the Persians, this anathema must have escaped the fury of the barbarian fires", ripreso ancora da J.E. Harrison addirittura quando era ormai noto il ripristino del monumento (Mythology and Monuments of Ancient Athens, being a translation of a portion of the "Attica" of Pausanias, by M. DE G. Verrall, with intr. and comm. by J.E. HarrRISON, 
l'originale, ${ }^{90}$ pensando che l'unico anathema arcaico fosse costituito soltanto dai ceppi (ed essendo in questo vivacemente contestato da $\mathrm{T}$. Bergk). ${ }^{91}$ Lo studioso si sarebbe poi ovviamente corretto una volta venuto alla luce il vero originale. ${ }^{92}$ L'affermazione che merita di essere sottolineata, invece, è che Erodoto avrebbe visto e trascritto la dedica nel 431 a.C. ${ }^{33}$

\subsection{3) ERODOTO, ATENE E LA REDAZIONE DELLE STORIE: SCHMITZ, KIRCHHOFF, STEIN}

La discussione in merito a un secondo soggiorno di Erodoto ad Atene ha come cardine precisamente il passo in cui descrive la nostra quadriga e si ravviva proprio in questi anni: lo stesso Kirchhoff, infatti, aveva appena pubblicato il suo saggio sulla data di stesura delle Storie di Erodoto ${ }^{94}$. Il problema, secondo J.E. Powell, era allora "in its infancy" ${ }^{\prime \prime 5}$, ma era in realtà già registrato nella sua complessità per esempio da L. Schmitz nel celebre dizionario biografico di W. Smith ${ }^{96}$ ed avrebbe continuato ad intrecciarsi con il dibattito topografico successivamente. Ecco come Schmitz riassumeva la questione della presenza di Erodoto ad Atene alla fine degli anni '30: "There is however a passage in his own work (5.77) from which we must in all probability infer, that

London 1890, 523-24). Meno ingenuamente C. BURSIAN, Uebersicht der neusten Leistungen und Entdeckungen auf dem Gebiete der griechischen Kunstgeschichte. Erster Artikel: die griechische Kunst bis zu den Zeiten des Pheidias, «Neue Jahr. für Philol. und Pädagogik», 26 Bd. 73-74 (1856), 421-441: 436, pur scrivendo prima del ritrovamento, si mostrava scettico in merito alla sopravvivenza della dedica alle distruzioni persiane e cominciava già ad ipotizzare per lo meno uno spostamento.

90 A. KIRCHHOFF, Über zwei attische Votivinschriften, 412; l'ipotesi era invece accettata da A. MichaELIS, Bemerkungen zur Periegese der Akropolis von Athen, «MDAI(A)», 2 (1877), 85-106: 97.

91 T. BergK, Poetae Lyrici Graeci, III, Lipsiae 18824, 477-79.

92 KirchHOFf (LOLLING), Bemerkungen zu dem Bruchstück, 111-12.

93 A. KIRCHHOFF, Über zwei attische Votivinschriften, 410.

94 A. KIRCHHOFF, Über die Abfassungszeit des Herodotischen Geschichtswerkes, Berlin 1868 (Abhandlungen der Königlichen Akademie der Wissenschaften zu Berlin). 95 J.E. POwell, The History of Herodotus, Cambridge 1939, VII-VIII.

96 L. Schmitz, Herodotus, in A Dictionary of Greek and Roman Biography and Mythology, II, ed. by W. SMITH, London 1849, 431-36: 431, con bibliografia, in particolare F.C. Dahlmann, Herodot. Aus seinem Buche sein Leben, Altona 1823. 
in B. C. 431, the year of the outbreak of the Peloponnesian war, he was at Athens; for it appears from that passage that he saw the Propylaea, which were not completed till the year in which that war began". ${ }^{97}$ Vale a dire che secondo tutta una scuola di pensiero, ben rappresentata dalle prime edizioni dell'autorevole commento erodoteo di Stein, i Propilei cui si riferisce Erodoto erano la famosa costruzione di Mnesicle e, dato che essi erano stati notoriamente costruiti fra 437 e 432, ciò costituiva un chiaro terminus post quem per il passo erodoteo. ${ }^{98}$

\subsection{4) IL MEGARON VOLTO AD OCCIDENTE}

Già L. Ross, tuttavia, aveva individuato i resti di Propilei precedenti a quelli di Mnesicle, e aveva anzi ipotizzato che i blocchi, riportanti tracce di un incendio e una serie di fori, potessero corrispondere proprio alle "mura bruciacchiate dal fuoco acceso dal Medo, davanti al megaron volto ad Occidente", alle quali dovevano essere appesi i ceppi dei prigionieri Beoti e Calcidesi (Her. V 77). ${ }^{99}$ Inoltre Ross riteneva che il megaron volto ad Occidente potesse essere l'atrio occidentale degli Antichi Propilei. Le identificazioni a quei tempi più accreditate erano invece la cella occidentale del Partenone ${ }^{100}$ oppure la cella occidentale dell'Eretteo, ${ }^{101}$ interpretazione quest'ultima che prevalse, via via precisandosi come riferimento alla cella occidentale del Tempio arcaico di Atena,${ }^{102}$ poi scavato da W. Dörpfeld. ${ }^{103}$

97 Schmitz, Herodotus, 431.

98 Herodotos, erkl. von H. STEIN, I, Berlin 1856, XXIII; XLIII (= Berlin 1864², 23 n. $2 ; 45=$ Berlin $1870^{3}$, XXIII n. 2 ; XLV = Berlin $1877^{4}$, XXIII n. 2; XLVI). Per la diversa proposta delle ultime due edizioni v. par. 3.1.16.

99 L. Ross, Archäologische Aufsätze, I, Leipzig 1855, 78-81. L'ipotesi di Ross verrà corretta da W.B DinsmoOR JR., The Propylaia to the Athenian Akropolis, I, The Predecessors, Princeton NJ 1980, 5; 43 n. 25; 61: v. par. 3.2.12.

100 Per esempio K. BÖTTICHER, Ueber agonale Festtempel und Thesauren, deren Bilder und Ausstattung, «Philologus», 17 (1861), 577-605: 577 n. 1; A. KIRCHHOFF, Über zwei attische Votivinschriften, 412; Herodotos, erkl. von H. STEIN, III, Berlin 1859, 73.

101 Per esempio K.L. KAYSER, Beiträge zur Kritik des Pausanias, «RhM», N.F. 5 (1847), 347-68: 364.

102 Per esempio BuRSIAN, Uebersicht der neusten Leistungen und Entdeckungen, 436; A. MiCHAELIS, Bemerkungen zur Periegese der Akropolis von Athen, «MDAI(A)», 2 (1877), 85-106: 102. Ma v. anche par. 3.2.12.

103 W. DÖRPFELD, Der alte Athena-Tempel auf der Akropolis zu Athen, 


\subsection{5) TERMINOLOGIA: TEMPIO ARCAICO DI ATENA, HEKATOMPEDON, PROPILEI}

E' bene a questo punto aprire una breve parentesi, per ricorrere a studi riassuntivi più recenti: è infatti necessario chiarire che nella bibliografia riguardante la quadriga per la vittoria ateniese sui Beoti e sui Calcidesi si usano spesso come sinonimi "Tempio arcaico di Atena" e "Hekatompedon" a proposito del "megaron volto ad Occidente" di cui parla Erodoto, di fronte al quale avrebbe dovuto trovarsi il muro da cui pendevano i ceppi dei prigionieri. Spiegava invece, ad esempio, già I. Thallon Hill che Tempio arcaico di Atena e Hekatompedon andrebbero distinti, anche se alcuni studiosi utilizzano indifferentemente entrambi i nomi, oltre al nome di Opisthodomos e persino di Eretteo intendendo come predecessore di quest'ultimo il Tempio arcaico di Atena, dato che si ergeva in un'area parzialmente coincidente con quella in seguito occupata dall'Eretteo. ${ }^{104}$ Anche J. Travlos insisteva nel sottolineare che "the Hekatompedon is not identical with and, consequently, should not be confused with the Old Temple of Athena", ${ }^{105}$ dato che il termine Hekatompedon viene pure frequentemente utilizzato per indicare un predecessore del Partenone sul sito del Partenone (ove è attestata una serie di templi), ${ }^{106}$ in particolare un grande tempio in poros che sarebbe stato costruito fra 570 e 566 a.C. e che si pensa dedicato ad Atena in occasione della celebrazione delle prime Panatenee, tempio soprannominato anche "Ur-Parthenon" dagli archeologi tedeschi e "grandfather of the Parthenon" da W.B. Dinsmoor; il Tempio arcaico

«MDAI(A)», 10 (1885), 275-77; DÖRPFELD, Der alte Athena-Tempel auf der Akropolis zu Athen, «MDAI(A)», 11 (1886), 337-51. Ulteriore bibliografia in J. Travlos, Pictorial Dictionary of Ancient Athens, London 1971, 143; v. ora M.C. MonACO, Il Tempio arcaico e il grande altare di Atena Polias, in E. GRECO, Topografia di Atene, Atene-Paestum 2010, 126-28.

104 I. Thallon Hill, The Ancient City of Athens. Its Topography and Monuments, Cambridge MA 1953, 136-38.

105 Travlos, Pictorial Dictionary, 258, con bibliografia.

106 Sull'Antico Partenone e il Partenone v. brevemente Travlos, Pictorial Dictionary, 444-45 con bibliografia; cfr. B. Holtzmann, L'acropole d'Athènes, Paris 2003, 83-144; v. ora anche R. DI CESARE, Lo stereobate sotto il Partenone e l'Architettura H, in Greco, Topografia di Atene, 96-101 e M.C. Monaco, Il Partenone, in Greco, Topografia di Atene, 101-08. 
di Atena, invece, "lies between the Parthenon and the Erechteion": ${ }^{107}$ delle possibili fasi dell'esistenza del tempio l'ultima risalirebbe all'epoca pisistratide (anni '20 del VI secolo) o ai primordi della democrazia. ${ }^{108}$

In realtà, come emerge chiaramente dalla sintesi di B. Holtzmann, ${ }^{109}$ sull'uso del termine Hekatompedon si sono confrontati i sostenitori delle due principali tesi ricostruttive di questa zona dell'Acropoli: la tesi unitaria prevede infatti diverse fasi di un unico tempio dedicato ad Atena Polias, identificato quindi anche con la cosiddetta architettura $\mathrm{H}$, richiamante nel nome, ancora una volta, l'Hekatompedon; ${ }^{110}$ è invece la tesi dualista ad attribuire il nome dell'Hekatompedon e parte dei resti dell'architettura $\mathrm{H}$ ad un predecessore del Partenone, un tempio di Atena Parthenos che sarebbe stato già costruito in età arcaica quasi in contrapposizione con quello di Atena Polias. ${ }^{111}$ Sarebbe infine possibile anche interpretare il termine "Hekatompedon" in modo semplicemente etimologico, come spazio di cento piedi sull'Acropoli, non necessariamente coincidente con un tempio. ${ }^{112}$

107 Travlos, Pictorial Dictionary, 143, con bibliografia.

108 Cfr. Monaco, Il Tempio arcaico, 126-27 per le diverse proposte di datazione; discussione in S. Angiolillo, Arte e cuultura nell'Atene di Pisistrato e dei Pisistratidi, Bari 1997, 63-64.

109 Holtzmann, L'acropole d'Athènes, 75-81, con ampia bibliografia.

110 T. WiEgand, Die archaische Porosarchitektur der Akropolis zu Athen, CasselLeipzig 1904, 1-72; W.H. Plommer, The Archaic Acropolis: Some Problems, «JHS», 80 (1960), 127-59; I. BEYER, Die Reliefgiebel des Alten Athena-Tempels der Akropolis, «AA», 1974, 639-51; ID., Die Datierung der grossen Reliefgiebel des Alten AthenaTempels der Akropolis, «AA», 1977, 44-74.

111 R. Heberdey, Altattische Porosskulptur, Wien 1919; W.B. DinsmoOr, The Hekatompedon of the Athenian Acropolis, «AJA» 51 (1947), 109-51 + tav. XXVII-

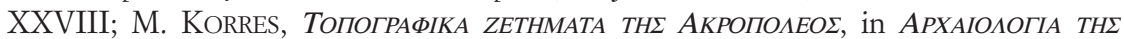

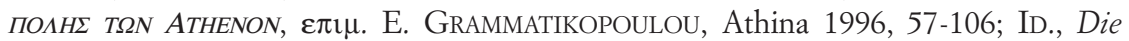
Athena-Tempel auf der Akropolis, in Kult und Kultbauten auf der Akropolis. Internationales Symposion vom 7. bis 9. Juli 1995 in Berlin, hrsg. von W. HOEPFNER, Berlin 1997, 218-43.

112 F. PreißHofen, Zur Topographie der Akropolis, «AA», (1977), 74-84: 79-80 riprende il significato di "Bezirk" proposto già da W. DitTEnberger, Zur Hekatompedon-Inschrift, «Hermes», 26 (1891), 472-73 lasciandone aperta l'identificazione; a questo proposito R. TÖLLE-KastenbeIn, Das Hekatompedon auf der Athener Akropolis, «JDAI» 108 (1993), 43-81, ha pensato all'area su cui sarebbero sorti in seguito Pre-Partenone e Partenone. 
M. Korres ha peraltro proposto di eliminare completamente il termine dall'uso corrente, per sostituirlo con i più perspicui "Tempio altoarcaico di Atena Poliade", "Partenone arcaico" (sul quale però, come abbiamo visto, si discute) ${ }^{113}$ e "Tempio tardo-arcaico di Atena Poliade" (o archaios naos). ${ }^{114} \mathrm{E}$ ' a quest'ultimo tempio che si farà qui costantemente riferimento; per esigenze di spazio, tuttavia, la definizione di "Tempio tardo-arcaico di Atena Poliade" verrà ridotta, in relazione alla collocazione topografica della quadriga e/o dei ceppi, alla forma abbreviata di "Tempio arcaico di Atena", naturalmente senza che questo significhi un'adesione pregiudiziale ad una particolare tesi ricostruttiva, ma semplicemente perché definizione non così equivoca e ormai affermatasi nella bibliografia relativa alla quadriga.

Complesse sono anche le vicende dei Propilei, che costituiscono l'altro punto di riferimento nella discussione topografica relativa alla quadriga, localizzata da Erodoto "alla mano sinistra per chi entra nei propilei dell'Acropoli". Prima dei famosi Propilei di Mnesicle, ${ }^{115}$ eretti a partire dal 437 a.C., esisteva già, naturalmente, un'entrata monumentale dell'Acropoli. Erodoto avrebbe visto proprio questa, che prende il nome di "Propylon" o "Antico Propylon". Sulla data di costruzione non c'è accordo; per W.B. Dinsmoor jr. si possono individuare tre fasi: la prima poco dopo la I Guerra Persiana, la seconda poco prima della II, la terza in epoca cimoniana, intorno al 460 a.C. $\operatorname{circa}^{116}$ (ma la prima monumentalizzazione dell'area potrebbe risalire al 566 a.C.). ${ }^{117}$

113 Sulla questione, v. i già citati HolTzMANn, L'acropole d'Athènes, 75-81 e Di Cesare, Lo stereobate sotto il Partenone e l'Architettura H, 96-101.

114 M. KORRES, Architettura classica ateniese, in Atene e la Magna Grecia dall'età arcaica all'Ellenismo. Atti del quarantasettesimo convegno di studi sulla Magna Grecia. Taranto, 27-30 Settembre 2007, Taranto 2008, 17-46: 17-23.

115 Sui quali v. W.B. DinSMOOR - W.B. DinSMOOR JR. - A. NORRE DinSMOOR, The Propylaia to the Athenian Akropolis, II, The Classical Buildings, Princeton NJ 2004; v. anche Travlos, Pictorial Dictionary, 482-92, con foto, ricostruzioni (in particolare 486 n $^{\circ}$ 611), bibliografia e HolTzMAnN, L'acropole, 145-57.

116 DinsmoOR JR., The Propylaia, I, XVII-XVIII.

117 Travlos, Pictorial Dictionary, 482-83 con bibliografia; in generale v. HoltzMAnN, L'acropole, 70-72, con sintesi delle proposte di ricostruzione alternative (cfr. specialmente H. EITELJORG II, The Entrance to the Athenian Acropolis Before Mnesicles, Boston 1993 (Archaeological Institute of America, Monograph New Series 1); ID., Revisiting the Pre-Mnesiklean Entrance to the Athenian Acropolis, «AJA», 115 (2011), 641-45 e I. Mylonas SHEAR, The Western Approach to the Athenian Akropolis, «JHS», 119 (1999), 86-127). Cfr. anche M.C. MonaCO, I propilei e la Pinacoteca, in GreCO, Topografia di Atene, 81. 


\subsection{6) A QUALI PROPILEI SI RIFERIVA ERODOTO? ROSS, BURSIAN, KIRCHHOFF, WEIZSÄCKER}

Nel dibattito sulla quadriga rimaneva appunto da stabilire quali Propilei avesse in mente Erodoto. All'inizio di questa prima fase di studi la proposta di Ross, che Erodoto si riferisse direttamente agli Antichi Propilei (chiamandoli megaron) o in alternativa ne vedesse comunque le rovine (le mura bruciacchiate) gode di scarsa approvazione. ${ }^{118}$ Per Bursian, in particolare, era impensabile che un Greco potesse definire megaron dei Propilei. ${ }^{119}$ L'ipotesi era tuttavia seguita almeno in parte da T.H. Dyer, che relativamente all'esposizione dei ceppi pensa però alle mura esterne dell'Acropoli. ${ }^{20}$ Ancora nel 1878, invece, Kirchhoff utilizzava il nostro passo erodoteo come certissimo punto di riferimento per stabilire senza ombra di dubbio che Erodoto si era trovato ad Atene dopo il 432, poiché la precisa descrizione topografica dimostrava che lo storico era testimone oculare del completamento dei Nuovi Propilei. ${ }^{121}$

All'opposto, un'elaborazione originale dell'idea di Ross, che Erodoto cioè conoscesse gli Antichi Propilei, venne formulata (naturalmente senza l'identificazione dei Propilei con il megaron) da P. Weizsäcker: "Nun spricht zwar Herodot noch von den vorperikleischen Propyläen, denn er weiss ja noch von den medischen Brandspuren an den Mauern zu berichten"; soltanto la frase successiva, che menziona espressamente i Propilei, sarebbe stata scritta dopo l'intervento di Mnesicle: difatti qui Erodoto parla "in der Gegenwart", mentre nella frase precedente, relativa ai ceppi e alle mura annerite dall'incendio persiano, "in der Vergangenheit". ${ }^{122}$

\subsection{7) LA QUADRIGA ALL'INTERNO DEI PROPILEI? WEIZSÄCKER}

Quanto all'esatta collocazione della quadriga, Weizsäcker inter-

118 L. PRELLER, Zur archaeologischer Litteratur, «Neue Jahr. für Philol. und Pädagogik», 26 Bd. 73-74 (1856): 73-82: 76.

119 BURSIAN, Uebersicht der neusten Leistungen und Entdeckungen, 436.

120 DYER, Ancient Athens, 132.

121 A. KIRCHHOFF, Über die Entstehungszeit des Herodotischen Geschichtswerkes. Zwei akademische Abhandlungen, Berlin 1878², 16-17.

122 P. WeIzsäcker, Die Aufstellung der Bildwerke in den Propyläen zu Athen, «Arch. Zeitung», 32 N.F. 7 (1874), 110-11: 110. 
pretava il controverso passo di Erodoto "in den Propyläen beim Eintreten links, also auf der nördlichen Seite": insomma, la quadriga si sarebbe "indubitabilmente" trovata all'interno dei Propilei pre-mnesiclei - peraltro intesi come molto simili a quelli di Mnesicle - a sinistra entrando secondo quanto scrive Erodoto, quindi nella parte Nord dell'atrio orientale dei Propilei. ${ }^{123}$ Pausania avrebbe infatti semplicemente visto la quadriga subito dopo la Promachos uscendo dall'Acropoli. ${ }^{124}$ Nessun contrasto veniva quindi rilevato fra le due fonti.

\subsection{8) DEL TESTO DI ERODOTO, SE SIA O NO DA CORREGGERE: E IIONTI O EEIONTI? WACHSMUTH, SCHÖLL}

E' a questo punto che irrompe nel dibattito C. Wachsmuth: con il suo intervento, seppure eccessivamente drastico nelle conclusioni, ha il merito di aver condotto in prima persona o comunque stimolato opportune riflessioni linguistiche, oggi purtroppo trascurate. Egli escludeva che "die mächtige Quadriga" potesse oggettivamente trovare spazio all'interno dei Propilei e proponeva di conseguenza di correggere il

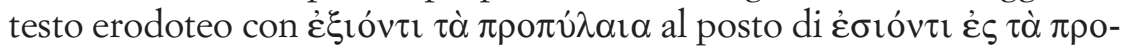
$\pi v ́ \lambda \alpha \iota \alpha$, in modo che la quadriga venisse a trovarsi sempre a sinistra, ma uscendo dai Propilei. ${ }^{125} \mathrm{La}$ correzione si giovava del confronto con



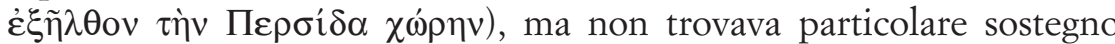
nella tradizione manoscritta. Ancor meno ne trovò presso gli studiosi contemporanei, alcuni dei quali ne travisarono gli intendimenti.

R. Schöll, ad esempio, attribuiva alla volontà di Wachsmuth di concordare i passi di Erodoto e Pausania la correzione del primo a vantaggio del secondo, ${ }^{126}$ laddove era chiaro a entrambi gli studiosi come in questo passo Pausania potesse distaccarsi dal suo abituale ordine topografico: Schöll parla di un possibile "sachliches Motiv" alla base della menzione ravvicinata della Promachos e della quadriga, ${ }^{127}$ Wachsmuth

123 WeIZSÄCKER, Die Aufstellung der Bildwerke, 110.

124 WeIZsäCKER, Die Aufstellung der Bildwerke, 111.

125 C. Wachsmuth, Die Stadt Athen im Alterthum, I, Leipzig 1874, 150 n. 2.

126 R. SCHÖLl, recensione a C. WACHSMuth, Die Stadt Athen im Alterthum, Leipzig 1874, «Jenaer Literaturzeitung», 2 (1875), 684-691: 686. Similmente W. JudEICH, Topographie von Athen, München 1905, 215-16 n. 8 (= München 193122, 236).

127 SCHÖLL, recensione a C. WACHSMUTH, 686. 
ritornerà sull'argomento sottolineando che Pausania introduce la descrizione dei due monumenti proprio con un'eccezione (X ӧ $\sigma \alpha \kappa \alpha \tau \dot{\varepsilon} \lambda \varepsilon \xi \alpha) .{ }^{128}$

\subsection{9) ANCORA DELLA QUADRIGA ALL'INTERNO DEI PROPILEI: BURSIAN, WEIZSÄCKER}

Da una tale eccezione K. Bursian si sentiva autorizzato a concludere che la quadriga potesse trovarsi nell'atrio occidentale dei Propilei. ${ }^{129}$ Le dimensioni della quadriga non preoccupavano poi Weizsäcker, che per ragioni di simmetria (corrispondenza con altre opere d'arte) continuava a sostenere una collocazione del monumento nell'atrio orientale (lato Nord). ${ }^{130} \mathrm{Ma}$ il problema di queste ultime due proposte è, innanzitutto, di andare ad occupare in modo troppo ingombrante spazi destinati ad un passaggio ordinato di carri, cavalli, cortei. ${ }^{131}$ $\mathrm{Si}$ aggiunge poi un'obiezione ancor più generale: gli anathemata dedicati nei santuari sono di solito eretti all'esterno degli edifici. ${ }^{132}$

128 C. WaChSMUTH, Der Standort des ehernen Viergespann auf der Akropolis von Athen, «Neue Jahr. für Philol. und Pädagogik», 49 Bd. 119, I (1879), 18-24: 18.

129 K. Bursian, Alterthumskunde, recensione a C. WaChSMUth, Die Stadt Athen im Alterthum, Leipzig 1874, Fr. AdLER, Die Stoa des Königs Attalos II zu Athen, Berlin 1875, Aug. Schulz, De Theseo, Breslau 1874, «Liter. Centralblatt für Deutschland», (1875), 1079-1084: 1080.

130 P. WeIzsäcker, Zur Periegese des Pausanias, «Arch. Zeitung», 33 N.F. 8 (1875), 45-47.

131 E. CuRTIus, Zur Topographie der Propyläen, «Arch. Zeitung», 33 N.F. 8 (1875), 53-54: 54, a proposito della sola proposta di Weizsäcker; l'obiezione può essere estesa a quella di Bursian. Si aggiunga che i calcoli di MiCHAELIS, Bemerkungen zur Periegese, 96-97 escludevano la contemporanea presenza della quadriga, della statua di Pericle e dell'Atena Lemnia nell'atrio orientale Nord, come sostenuto da WEIZSÄCKER, Zur Periegese, 45-47. Quanto alla proposta di BunSIAN, Alterthumskunde, 1080, Wachsmuth si chiedeva se Pausania non avrebbe dovuto allora nominare la quadriga nel corso delle sue descrizioni all'ingresso nei Propilei (WaCHSMUtH, Der Standort des ehernen Viergespann, 18, seguito da W. MiLLER, A History of the Akropolis of Athens, «Amer. Journ. of Archaeology and of the History of the Fine Arts», 8 (1893), 473-556: 504-05: l'obiezione potrebbe avere peso minore supponendo che Pausania avesse volutamente tralasciato la quadriga per menzionarla insieme con la Promachos, in un raggruppamento di tipo tematico: in realtà non sembra essere così, v. par. 3.1.11, 3.2.10, 4; in ogni caso, resta il problema dell'ingombro).

132 B. Bergquist, The Archaic Greek Temenos, Lund 1967 (Acta Instituti 


\subsubsection{0) IL VALORE TEMPORALE DI E $\Sigma$ IONTI: LA QUADRIGA DINANZI AI PROPILEI? CURTIUS}

E. Curtius sollevava invece un'ulteriore questione, frutto del dibattito innescato a quei tempi dalla correzione di Wachsmuth: si tratta del

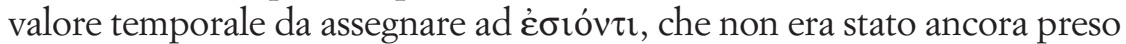
in considerazione e sul quale attualmente non ci si sofferma. Secondo Curtius, infatti, il possibile (come si sa) valore futuro di $\varepsilon \tilde{i} \mu$ t diventereb-

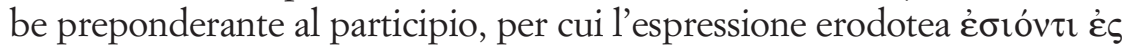
$\tau \grave{\alpha} \pi \rho 0 \pi v ́ \lambda \alpha \iota \alpha$ non potrebbe che significare "per chi è in procinto di entrare nei Propilei", cioè che la quadriga si sarebbe trovata immediatamente prima di essi. ${ }^{133}$ Emergeva qui per la prima volta l'idea che la quadriga potesse essere collocata al di fuori dell'Acropoli, un'idea che sarebbe stata rilanciata dagli studi successivi. Curtius rimarcava inoltre la necessità che l'importante anathema potesse essere visto da lontano, o comunque in una posizione di rilievo, dato che celebrava la prima impresa dell'Atene democratica: in questo senso erano scartate le collocazioni interne ai Propilei. ${ }^{134}$

Tuttavia l'aspetto più innovativo (anche se non condivisibile) dello studio di Curtius è costituito, come si diceva, dall'interesse linguistico: a questo proposito è citato come rivelatore un passo di Pausania (II 10, 2: descrizione del santuario di Asclepio a Sicione), in cui a $\pi \alpha \rho \varepsilon \lambda \theta 0 \tilde{\sigma l}$,

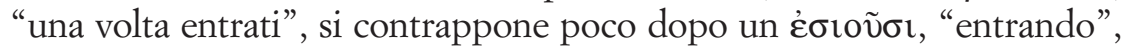
riferito a due statue che si trovavano ai lati dell'entrata. Di conseguenza la correzione di Wachsmuth, $\dot{\varepsilon} \xi$ ıóv $\tau$, finirebbe col riportare la quadriga all'interno dei Propilei, significando "per chi è in procinto di uscire".

\subsubsection{1) PAUSANIA E LA TOPOGRAFIA DELL'ACROPOLI: MICHAELIS}

La prima reazione fu quella di Michaelis, il quale, accettate le con-

Atheniensis Regni Sueciae $=$ Skrifter utgivna av Svenska Institutet i Athen, Series in $4^{\circ}$, 13), 106; KeEsLIng, The Votive Statues, 12. Se anche si volesse accettare per il Propylon la ricostruzione di EITELJORG II, The Entrance, 59-86, che non ne fa un vero e proprio edificio, una collocazione della quadriga al suo interno rimarrebbe poco opportuna, $e$ andrebbe comunque incontro (tolti i calcoli relativi ai soli Propilei mnesiclei) alle obiezioni già esposte alla nota precedente.

133 CuRTIus, Zur Topographie, 53-54.

134 CurTius, Zur Topographie, 54. 
siderazioni linguistiche di Curtius, non poteva però accoglierne le conclusioni topografiche. Egli osservava giustamente che a questo punto non sarebbe stato più spiegabile il testo di Pausania: il Periegeta, infatti, segnala molto esplicitamente quando si distacca dal consueto ordine topografico (v. per es. Paus. V 14, 4; 10, laddove spiega per ben due volte che descriverà gli altari di Olimpia nell'ordine in cui gli Elei li utilizzano e non in quello in cui sorgono); anche qualora considerassimo la frase $\chi \omega p i \varsigma \delta \dot{\varepsilon} \grave{\eta}$ ö $\sigma \alpha \kappa \alpha \tau \varepsilon \dot{\lambda} \boldsymbol{\varepsilon} \xi \alpha$ (Paus I 28, 2) come un segnale esplicito, avremmo ancora la statua di Pericle e l'Atena Lemnia, alle quali segue la descrizione delle mura dell'Acropoli; dunque quelle opere d'arte dovevano trovarsi almeno all'interno di esse (visto poi che la salita all'Acropoli era già stata descritta). ${ }^{135}$

\subsubsection{2) UNO SPOSTAMENTO DELLA QUADRIGA O UNO SPOSTAMENTO DEL PUNTO DI VISTA DI ERODOTO? MICHAELIS, WEIZSÄCKER}

All'analisi appena ricordata del passo di Pausania, tuttora condivisibile, Michaelis faceva seguire due proposte di soluzione: l'una, cui ricorrere "im äussersten Nothfalle" (come vedremo, "l'emergenza" sarebbe arrivata di lì a pochi anni), ipotizzava per la prima volta - ma senza convinzione - uno spostamento della quadriga tra l'età di Erodoto e quella di Pausania dovuto forse alla costruzione della scalinata romana dei Propilei; l'altra prendeva in considerazione l'espres-

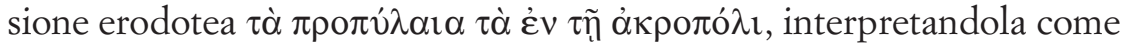
riferimento all'atrio orientale dei Propilei. A rigor di logica sarebbe in effetti possibile "entrare nei Propilei" sia da Ovest sia da Est, ed Erodoto, parlando di "propilei, quelli sull'Acropoli", contrapporrebbe questo atrio minore, che si trova proprio all'interno dell'Acropoli, all'atrio monumentale esterno, che avrebbe chiamato semplicemente "Propilei" ${ }^{136}$ Le ragioni di una prospettiva così insolita, dall'interno all'esterno, andrebbero ricercate nel fatto che Erodoto ha appena nominato i ceppi dei prigionieri beoti e calcidesi, ${ }^{137} \mathrm{i}$ quali si trovavano appunto all'interno dell'Acropoli, di fronte al Tempio arcaico di Atena

\footnotetext{
135 Michaelis, Bemerkungen zur Periegese, 95; 97-98.

136 MichaELIS, Bemerkungen zur Periegese, 98-99.

137 MichaELIS, Bemerkungen zur Periegese, 99-100.
} 
(il megaron cui si riferisce Erodoto, qui - V 77 - come a VIII 53). ${ }^{138} \mathrm{La}$ quadriga, per chi procedeva sull'antica strada ancora oggi in parte riconoscibile tra l'antico Tempio di Atena e i Propilei, sarebbe appunto da localizzare a sinistra, immediatamente prima dei Propilei, così conciliando le indicazioni di Erodoto e di Pausania (con le statue di Pericle e dell'Atena Lemnia conseguentemente a destra oppure all'interno dei Propilei). ${ }^{139}$ Una simile spiegazione, che potremmo definire "concordista", mutuando l'aggettivo da altri campi di studio, riuscirebbe addirittura ad armonizzare, oltre a Erodoto con Pausania, pure Erodoto con le successive indagini archeologiche: $i$ resti di una base nei pressi della Promachos rintracciati da Stevens, ${ }^{140}$ infatti, verrebbero a trovarsi proprio a sinistra procedendo dall'antico Tempio di Atena ai Propilei, anziché a destra procedendo nel più naturale senso opposto.

Purtroppo un problema della proposta di Michaelis sta proprio nell'innaturalità dell'indicazione erodotea se interpretata in questo senso: Erodoto avrebbe molto più semplicemente detto "uscendo dall'Acropoli", perché il movimento prospettato da Michaelis altro non è che un'uscita e non un'entrata. ${ }^{141}$ L'ipotesi godette comunque di una qualche fortuna e venne ripresa da Weizsäcker, il quale immaginava che Erodoto nel corso della narrazione si fosse idealmente spostato all'interno dell'Acropoli. ${ }^{142}$

\subsubsection{3) IL CONTRIBUTO DI DIODORO ALLA TOPOGRAFIA: WEIZSÄCKER, WACHSMUTH}

Weizsäcker (come pure in seguito Wachsmuth) ${ }^{143}$ trovava poi

138 PRELLER, Zur archaeologischer Litteratur, 76; MiCHAELIS, Bemerkungen zur Periegese, 101-102.

139 MiCHAELIS, Bemerkungen zur Periegese, 103.

140 V. par. 3.2.16.

141 H. STEIN, Jahresbericht über Herodot für 1879, «Jahresber. über die Fortschr. der class. Alterthumwiss.», 7 Bd. 17, I (1879), 87-99: 94; A. FurTwängLER, Meisterwerke der griechischen Plastik, Leipzig 1893, 15 n. 1: "wenn man ohne näheren Beisatz vom Hineingehen in ein Thor spricht, denkt jedermann «von aussen hinein», nicht umgekehrt”.

142 P. WeIzsäcker, Pausanias und die Bildwerke in den Propylaien, «Neue Jahr. für Philol. und Pädagogik», 56 Bd. 133, I (1886), 1-28: 6.

143 C. Wachsmuth, Athenai, in RE, suppl. I (1903), 159-219: 203. 
sostegno ad una collocazione interna all'Acropoli e non dinanzi ai Propilei anche nel sintetico passo di Diodoro (X 24, 3): $:^{144}$ ma qui biso-

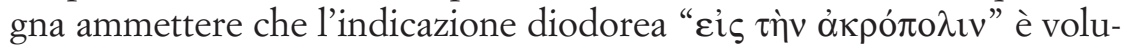
tamente generica (il brano è oltretutto parte degli estratti da Diodoro, gli "Excerpta Vaticana"), potrebbe derivare da una semplificazione dell'espressione erodote ${ }^{145}$ e quindi non avere un significato topografico autonomo. Ad ogni modo Weizsäcker riteneva anche strano che tra tutti i doni votivi che secondo Pausania si trovavano sull'Acropoli, ${ }^{146} \mathrm{gli}$ Ateniesi ne avessero voluto erigere uno dinanzi alle porte e considerava quindi, come non pochi altri, ${ }^{147}$ quella di Michaelis l'unica soluzione.

144 Testo: Diodori Bibliotheca Historica, II, post I. BEKKER et L. DindORF

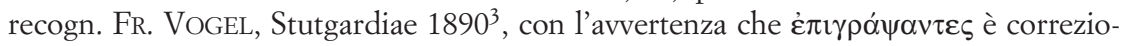
ne di H. van HERWERDEN, Spicilegium Vaticanum, Lugduni Batavorum 1860, a ypó $\psi \alpha v-$ $\tau \varepsilon \varsigma$, che è la lezione del manoscritto da lui decifrato (Codex Vaticanus Palimpsestus $n^{\circ}$ 73), ma non se ne vede la necessità, dal momento che anche $\gamma \rho \alpha \dot{\phi} \omega \omega$ può significare "iscrivere, incidere"; difatti C.H. Oldfather (Diodorus of Sicily, with transl. by C. H. OldFATHER, IV, Cambridge MA-London 1946, 92) non accoglie la correzione.

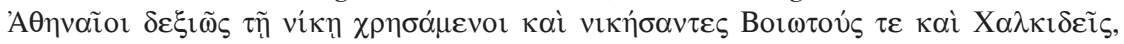

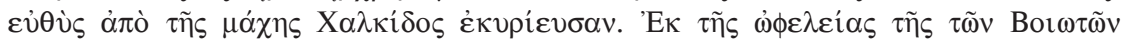

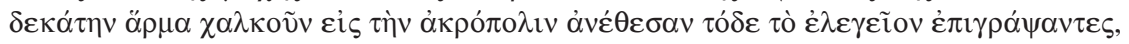

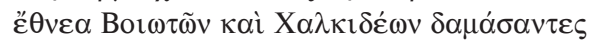

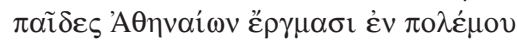

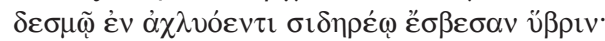

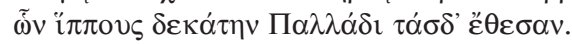

Gli Ateniesi fecero buon uso della loro vittoria e, avendo vinto i Beoti e i Calcidesi, subito dopo la battaglia si impadronirono della città di Calcide. Come decima del bottino di guerra strappato ai Beoti dedicarono un carro di bronzo sull'Acropoli, con iscritta la seguente elegia:

"Avendo i figli degli Ateniesi domato i popoli

dei Beoti e dei Calcidesi nelle imprese di guerra,

in una tetra catena di ferro ne spensero la tracotanza:

e come decima da essi posero queste cavalle a Pallade".

145 Per M. BoAs, De epigrammatis simonideis, Groningae 1905, 86 Erodoto sarebbe la fonte di Diodoro in ogni caso, "sive recta via sive per Ephorum".

146 Paus. V 21, 1.

147 V. p. es. A. MiLchHÖFER, Athen, in Denkmäler des klassischen Altertums zur Erläuterung des Lebens der Griechen und Römer in Religion, Kunst und Sitte, hrsg. von A. BAumeister, I, München-Leipzig 1885, 208, a sua volta ripreso alla lettera da A. Boetticher, Die Akropolis von Athen nach den Berichten der alten und den neuesten Erforschungen, Berlin 1888, 192. 
Essa fu tuttavia esclusa ${ }^{148}$ da ulteriori, opportune indagini linguistiche.

\subsubsection{4) LUUSO LINGUISTICO DI ERODOTO: WACHSMUTH, STEIN}

L'invito di Curtius ad approfondire l'uso linguistico di Erodoto venne difatti raccolto. Analizzando i passi in cui Erodoto dà indicazioni topografiche e confrontando anche esempi tratti da Pausania, Eliano e Luciano, Wachsmuth conclude che in questi casi il participio di $\varepsilon \tilde{i} \mu \mathrm{r}$ ha valore di presente, "per chi entra", quindi, non "per chi è in procinto" di farlo. L'oggetto si trova dunque proprio all'entrata, "sulla porta", non all'esterno. ${ }^{149}$ Molto chiaro in questo senso Her. I 51, 1: dei tripodi

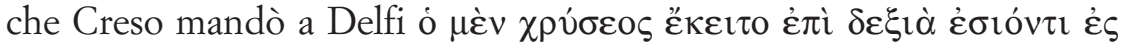

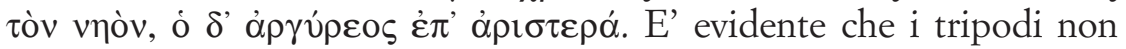
potevano trovarsi fuori dal tempio, bensì al suo interno, come sottolineato anche da Stein, che con ulteriori esempi erodotei conferma il valore preponderante di presente o anche di aoristo del participio in esame. ${ }^{150}$ L'analogia lessicale di I 51, 1 con Her. V 77 è notevole e dovrebbe almeno essere presa in considerazione da chi oggi ritiene assodato che Erodoto vedesse la quadriga dinanzi ai Propilei. Wachsmuth (seguito da Stein) scartava così le proposte di collocazione esterna (Curtius, Michaelis) perché linguisticamente impossibili, mentre le proposte di collocazione interna (Bursian, Weizsäcker) avevano già mostrato i loro limiti oggettivi.

\subsubsection{5) ANCORA DEL TESTO DI ERODOTO, SE SIA O NO DA

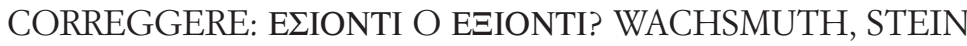

Di conseguenza il passo di Erodoto rimaneva per Wachsmuth corrotto e rendeva necessaria la sua correzione $\dot{\varepsilon} \xi$ ıóv $\tau$ : la quadriga si sarebbe trovata a sinistra uscendo nell'Acropoli dai Propilei, in direzione dell'Eretteo, ciò che non sarebbe neppure in contrasto con un'interpretazione "topografica" delle parole di Pausania. ${ }^{151}$ Siamo dunque ritornati al punto di partenza: l'area tra i Propilei e l'Eretteo costituiva,

148 Michaelis stesso non vi fa più cenno in O. JAHN - A. MichaELis, Arx Athenarum a Pausania descripta, Bonn $1901^{3}, 78$.

149 WaChSMUTH, Der Standort des ehernen Viergespann, 20-23.

150 STEIN, Jahresbericht über Herodot für 1879, 93.

151 WaChSMUTH, Der Standort des ehernen Viergespann, 24. 
ai primordi della ricerca, la naturale candidata ad ospitare la dedica per la vittoria sui Beoti e sui Calcidesi. ${ }^{152}$

Stein però, sebbene approvi i risultati linguistici di Wachsmuth specificando che $\varepsilon \tilde{\varepsilon} \mu \mathrm{t}$ ha valore di futuro all'indicativo regolarmente in Erodoto, ma soltanto valore di presente o di passato al participio, con

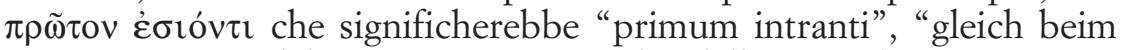
Eintritt" (come del resto suggerito anche dalla varia lectio $\pi \rho \tilde{\omega} \tau \alpha),{ }^{153}$ propone tuttavia una diversa soluzione a sostegno del testo tràdito. I Propilei andrebbero infatti intesi non tanto come un edificio, quanto come un atrio, dal quale si entra in un ambiente interno più che uscir-

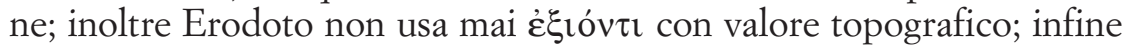
la iunctura $\dot{\varepsilon} \xi \xi \dot{\varepsilon} v \alpha \imath$ + accusativo proposta da Wachsmuth è d'uso raro e poetico. ${ }^{154} \mathrm{~A}$ proposito dell'ultima argomentazione si potrebbe ribattere con un esempio di Ateneo, ${ }^{155}$ in cui dal verbo $\varepsilon^{\prime} \xi$ lévol sembrerebbe

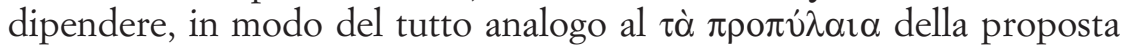
di Wachsmuth, l'accusativo $\tau \grave{\alpha} \varsigma \pi v ́ \lambda \alpha \varsigma$. In realtà, se si analizza l'intera

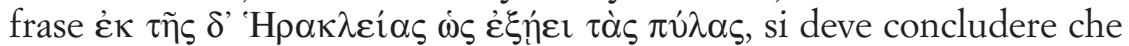
si tratta di un accusativo di relazione che precisa l'espressione più generica introdotta da $\dot{\varepsilon} \kappa$, che in Erodoto non c'è. ${ }^{156} \mathrm{Il}$ significato comune di $\dot{\varepsilon} \xi ı \varepsilon ́ v \alpha \imath+$ accusativo negli storici sembra peraltro quello di allontanarsi in marcia con l'esercito, ${ }^{157}$ un senso ovviamente inappropriato qui e comunque non riscontrabile altrove in Erodoto.

\subsubsection{6) GLI ANTICHI PROPILEI E LA TEORIA DELLO SPOSTAMENTO DELLA QUADRIGA; UN DIVERSO SIGNIFICATO DEL TERMINE ПPOПTАAIA: STEIN}

Dunque la soluzione (secondo Stein): Erodoto si riferiva agli

152 V. par. 3.1.1.

153 STEIN, Jabresbericht über Herodot für 1879, 93-94.

154 STEIN, Jahresbericht über Herodot für 1879, 94, seguito da E. BACHOF, Zu Herodotos, «Neue Jahr. für Philol. und Pädagogik», 52 Bd. 125, I (1882), 177-82: 178-79.

155 VIII 44 KAIBEL.

156 Ancora più fuori luogo mi sembra la citazione, da parte di L. WEBER, 5 rKA 'ET

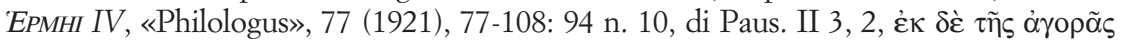


accusativo come un "uscire da", come sarebbe in teoria richiesto da Her V 77 corretto da Wachsmuth ("uscendo dai Propilei").

157 Cfr. A Greek-English Lexicon, by LIDDELL - SCOTT, rev. and augm. by JONES, with MCKENZIE, 589. 
Antichi Propilei, ${ }^{158}$ come già proposto da Ross, che però si era spinto un po' troppo oltre identificando con i Propilei anche "le mura bruciacchiate" o il "megaron" ${ }^{159}$ Per Stein, quindi, la quadriga si sarebbe trovata inizialmente all'interno degli Antichi Propilei, in accordo con l'uso linguistico erodoteo; in seguito alla costruzione dei Nuovi Propilei l'a-

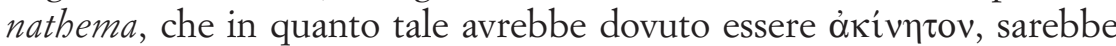
stato sì spostato, ma rimanendo all'interno dell'Acropoli-recinto sacro, dove la avrebbe poi vista Pausania, nei pressi della Promachos poco prima di lasciare l'Acropoli. La vicenda dello spostamento sarebbe simile a quella dei tripodi offerti da Creso a Delfi, che vennero trasferiti a causa dell'incendio del tempio di Apollo nel 548 a.C., rimanendo però in terra sacra. ${ }^{160}$

A ciò si potrebbe tuttavia obiettare che nel caso di Delfi si potevano invocare cause di forza maggiore (e quindi la volontà di salvare le dediche ad ogni costo) assenti dai lavori sull'Acropoli di Atene. Successivamente Stein avrebbe ulteriormente precisato la sua teoria, sostenendo la necessità di attribuire un valore "etimologico" alla parola $\pi \rho \circ \pi v \dot{\lambda} \alpha \iota \alpha$, con la quale Erodoto avrebbe voluto indicare non tanto l'edificio premnesicleo, quanto lo spazio antistante le porte $(\pi v ́ \lambda \alpha \imath)$, poi obliterato dalla costruzione mnesiclea. ${ }^{161} \mathrm{Ma}$ un simile significato non pare in genere attestato, ${ }^{162}$ tanto meno in Erodoto; ${ }^{163}$ Erodoto, inoltre, sta dando qui (V 77) un'indicazione topografica: il punto di riferimento dovrà quindi essere qualcosa di tangibile, non uno spazio un po' vago come risulterebbe da Stein. Ad ogni modo veniva così introdotta l'idea dello spostamento della quadriga, idea destinata ad essere sempre più

158 STEIN, Jahresbericht über Herodot für 1879, 95.

159 Ross, Archäologische Aufsätze, I, 78-81; v. par. 3.1.6.

160 STEIN, Jahresbericht über Herodot für 1879, 95.

XXIII n. 2).

161 Herodotos erkl. von H. STEIN, I.1, Berlin 18835, XXII n. 1 (= Berlin 19016,

162 Cfr. A Greek-English Lexicon, by LIDDELL - SCOTT, rev. and augm. by JONES, with MCKenZIE, 1496. Cfr. anche la discussione condotta sulle fonti da WeBER, IrKA 'ЕФ ЕРMHI, 101-02.

163 L'unico passo a prima vista conciliabile con il significato proposto da Stein -

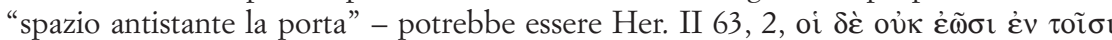


visto che al paragrafo precedente Erodoto si riferiva alla medesima situazione scriven-

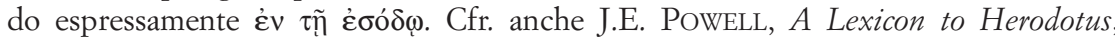
Cambridge 1938, 320. 
ripresa e perfezionata, sino a raggiungere il successo che ancora oggi riscuote.

Finirono ingiustamente dimenticate, invece, le indagini linguistiche: riportate da Bachof, ${ }^{164}$ sono poi citate (con approvazione) da A. Furtwängler ${ }^{165}$ e discusse per l'ultima volta da L. Weber. ${ }^{166}$ Ora, può anche essere che Erodoto in questo caso si esprimesse più approssimativamente, magari perché quel tipo di riferimento era per lui più familiare e comodo: non lo possiamo sapere, ma stupisce che chi prende molto sul serio l'indicazione erodotea per ricavarne una collocazione della quadriga esterna all'Acropoli non abbia più sentito il bisogno di confrontarsi con il dato linguistico, o almeno di menzionarlo per minimizzarne il peso.

\subsubsection{7) ERODOTO E LA TOPOGRAFIA: UN'AGGIUNTA PERSONALE IN ATENE O NOTIZIE RIFERITE? BAUER, BACHOF}

Chi invece cominciava ormai a nutrire seri dubbi sull'attendibilità topografica del passo di Erodoto era E. Bachof: ${ }^{167}$ egli polemizzava in particolare con Kirchhoff, che, come si è visto, ${ }^{168}$ utilizzava Her. V 77 come prova del ritorno di Erodoto ad Atene all'inizio della Guerra del Peloponneso, in quanto un riferimento così preciso alla quadriga sarebbe stato possibile soltanto ad un testimone oculare, ${ }^{169} \mathrm{ma}$ anche con A. Bauer, che aveva riproposto l'idea, in nuce già di Weizsäcker, ${ }^{170}$ di un'aggiunta successiva, per cui Erodoto avrebbe in un primo momento ricordato soltanto i ceppi, mentre avrebbe parlato della quadriga in occasione del suo (ipotizzato) secondo soggiorno ad Atene, localizzandola con riferimento ai Nuovi Propilei. ${ }^{171}$

164 BACHOF, Zu Herodotos, 178-79.

165 FURTWÄNGLER, Meisterwerke, 14 n. 6.

166 WEBER, IYKA 'EФ EPMHI, 94-95.

167 BACHOF, Zu Herodotos, 177-82.

168 V. par. 3.1.6.

169 KIRCHHOFF, Über die Entstehungszeit, 17.

170 WeIzsÄCKER, Die Aufstellung der Bildwerke, 110 notava infatti come Erodoto scrivesse al passato relativamente ai ceppi e utilizzasse invece un presente (per la precisione un perfetto resultativo con valore di presente) per ricordare la quadriga. V. par. 3.1.6.

171 A. BAUER, Themistokles. Studien und Beiträge zur griechischen Historiographie und Quellenkunde, Merseburg 1881, 16 n. 1. Impensabile, per Bauer, che Erodoto vedesse la quadriga negli Antichi Propilei: lo studioso presumeva infatti per questi ultimi una costruzione anteriore al 480 a.C. 
Secondo Bachof, al contrario, non era affatto un dato sicuro che

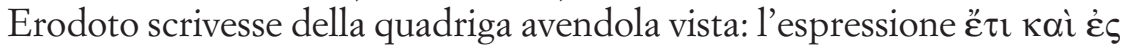
$\dot{\varepsilon} \mu \grave{\varepsilon} \tilde{\eta} \sigma \alpha \nu \pi \varepsilon \rho \iota \varepsilon o \tilde{v} \sigma \alpha \iota$ potrebbe così riguardare i soli ceppi osservati nel periodo trascorso ad Atene prima di imbarcarsi per Turi; Erodoto avrebbe poi anche potuto menzionare (ma non necessariamente come un'aggiunta di una successiva redazione) la quadriga con un modo di dire topografico molto comune, che non costituirebbe di per sé una prova di conoscenza diretta dei Nuovi Propilei. ${ }^{172}$ Che si trattasse dei Propilei di Mnesicle, sembrava assai più probabile allo studioso, anche perché, se non si teneva in considerazione l'ipotesi del rifacimento già esposta ma inizialmente scartata da Kirchhoff, ${ }^{173}$ si doveva presupporre che la quadriga fosse rimasta intatta presso gli Antichi Propilei; in ogni caso mostrando Erodoto di conoscere avvenimenti degli anni ' $30,{ }^{174}$ egli avrebbe dovuto almeno sentir parlare dei Nuovi Propilei, ormai i Propilei per antonomasia in quegli anni. Proprio per sentito dire Erodoto riferirebbe la posizione della dedica, ciò che spiegherebbe le difficoltà riscontrate nel passo ed il dibattito suscitato.

Con le proposte di Bauer e di Bachof, anch'esse destinate ad essere riprese (seppure non citate espressamente, ne verranno riprese le tematiche, ma spesso anche le conclusioni), il quadro degli studi sulla quadriga anteriori alla scoperta dell'unico frammento dell'iscrizione originaria può dirsi completo. Come si vede, erano state poste tutte le basi per le indagini successive, che in molti casi hanno approfondito temi già presenti nelle ricerche anteriori, talvolta però non tenendo conto dei risultati raggiunti.

\subsection{IL DIBATTITO DAL 1887}

\subsection{1) LA SCOPERTA DEL FRAMMENTO DELL'ISCRIZIONE ORIGINALE, IL RIFACIMENTO DELLA QUADRIGA ALL'ESTERNO DELL'ACROPOLI E IL MUTATO ORDINE DEI VERSI NELLA DEDICA: KIRCHHOFF}

A questo punto giunge il ritrovamento dell'iscrizione di VI seco-

172 BACHOF, Zu Herodotos, 177: qui Bachof cita il caso dei tripodi di Creso a Delfi, dei quali è segnalata anche la collocazione antecedente all'incendio del 548 a.C., di conseguenza riferita.

173 A. KIRCHHOFF, Über zwei attische Votivinschriften, 412. V. par. 3.1.2.

$174 \mathrm{Su}$ questo cfr. bibliografia in nn. 232, 235, 376. 
lo: l'annuncio è dato ancora una volta da Kirchhoff, che riconosce senza problemi di avere in passato escluso, delle sue due ipotesi, quella giusta. ${ }^{175}$ L'ipotesi del rifacimento è qui ampliata alla luce del dibattito precedente e prevede 1) l'originaria collocazione della quadriga sull'Acropoli nei pressi del muro eretto per i ceppi, la cui menzione all'inizio dell'epigramma secondo l'ordine originale appare quanto mai opportuna, quasi deittica potremmo dire; 2) la sua sottrazione o distruzione ad opera dei Persiani, con i frammenti della base poi riutilizzati altrove; 3) la ricostruzione una trentina d'anni più tardi, in un luogo diverso, quindi non più in collegamento con i ceppi, a sinistra prima di entrare nei Propilei ("linker Hand vor dem Eingange"), dei quali si può perciò dubitare, se si tratti già di quelli di Mnesicle; non vi sarebbero indicazioni successive sul luogo in cui la dedica era esposta. ${ }^{176}$ E' evidente quindi che Kirchhoff non attribuiva valore topografico al passo di Pausania; degli studi antecedenti mostrava comunque di aver accolto il dibattito intorno ai Propilei (da Ross ${ }^{177}$ a Stein, ${ }^{178}$ anche se non citati), ma non l'indagine linguistica. In questo modo, però, la nuova acquisizione epigrafica, di per sé fondamentale, veniva così ad oscurare un aspetto della ricerca. La variazione del luogo di esposizione, una volta accertato il ripristino del monumento, discendeva da una premessa qui non adeguatamente discussa: la collocazione della quadriga rifatta all'esterno dell'Acropoli ("vor dem Eingange"), un concetto, quello di una collocazione esterna, espresso per primo da Curtius riguardo però all'originale. ${ }^{179}$

Il nuovo ritrovamento consentiva peraltro di apprezzare nell'epigramma una diversa disposizione dei versi, con l'inversione dell'ordine degli esametri nella coppia di distici elegiaci; Kirchhoff formulava due ipotesi, senza prendere posizione: casuale inesattezza della copia o conseguenza di una riflessione consapevole. ${ }^{180} \mathrm{Ma}$ era in realtà quest'ultima l'ipotesi che poteva corroborare l'idea che la quadriga rinnovata fosse

175 KirCHHOFF (Lolling), Bemerkungen zu dem Bruchstück, 111-12.

176 KirChHOFf (Lolling), Bemerkungen zu dem Bruchstück, 113-14.

177 Ross, Archäologische Aufsätze, I, 78-81; v. par. 3.1.4 e 3.1.6.

178 STEIN, Jabresbericht über Herodot für 1879, 95; v. par. 3.1.16.

179 CuRTIUS, Zur Topographie, 53-54, v. par. 3.1.10. Certo Kirchhoff amplia la prospettiva coll'analisi del nuovo ritrovamento, in una direzione che risulterà gradita a Curtius, che quindi la riprenderà, questa volta riferendosi al ripristino della quadriga (E. CurTius, Die Stadtgeschichte von Athen, Berlin 1891, 155).

180 KirchHoff (LolLing), Bemerkungen zu dem Bruchstück, 114. 
stata collocata in un luogo diverso dall'originale: il riferimento ai ceppi in

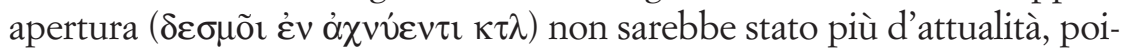
ché la quadriga ne era ormai lontana. ${ }^{181}$

\subsection{2) LA COLLOCAZIONE DELLA QUADRIGA ALL'ESTERNO DELL'ACROPOLI E IL CONFRONTO CON LE STATUE DEI CAVALIERI DI LYKIOS: CURTIUS}

La ricostruzione di Kirchhoff si diffuse anche grazie alla novità del ritrovamento epigrafico e fu quindi subito ripresa. ${ }^{182} \mathrm{Di}$ conseguenza l'idea che la dedica fosse stata innalzata dinanzi ai Propilei acquistava una forza tale da sembrare quasi un dato di fatto: Curtius, ricollegandola come già Stein alla costruzione dei Propilei di Mnesicle, ${ }^{183}$ ne deduceva una totale riorganizzazione dei monumenti, di cui la quadriga era l'esempio più notevole. ${ }^{184}$ Lo spazio antistante ai Propilei avrebbe garantito maggiore visibilità alla quadriga, sottraendola all'affollarsi di dediche all'interno dell'Acropoli. ${ }^{185}$ Nessun problema solleverebbe poi Pausania, visto che Curtius era fra quanti individuavano a I 28, 2 un momentaneo distacco dall'ordine topografico. ${ }^{186}$ Egli riteneva anche di aver rintracciato un altro esempio di spostamento di dediche nella vicenda per lui analoga delle statue dei cavalieri opera di Lykios descritte da Pausania (I 22,4) al suo ingresso nei Propilei. ${ }^{187}$ Non è qui il caso di riprenderne la storia in tutta la sua complessità ${ }^{188} \mathrm{ma}$, anche accettando la ricostruzione che attualmente gode dal punto di vista archeo-

181 Sono state proposte altre spiegazioni. V. par. 3.2.17.

182 V. già F. BAumgarten, Ein Rundgang durch die Ruinen Athens, Leipzig 1888, 55 (non ancora, invece, BoETticher, Die Akropolis von Athen, 192, che si limitava a riportare le parole di MILCHHÖFER, Athen, 208).

183 STEIN, Jahresbericht über Herodot für 1879, 95.

184 CuRTIUs, Die Stadtgeschichte, 154-55.

$185 \mathrm{Ma}$ sulla correttezza in un contesto sacro di un simile ragionamento, che fa prevalere una sorta di legame "decorativo" tra architettura e scultura v. par. 3.2.4, 3.2.11, 3.2.18, 4 .

186 CurTIUs, Die Stadtgeschichte, 300.

187 Curtius, Die Stadtgeschichte, 155.

188 Per un orientamento v. Pausania. Guida della Grecia, I, a c. di BESCHI Musti, 340-41; IG I3 511. Una sintesi delle proposte di datazione in N. ModenesI, Pausania "epigrafista" nell'itinerario della "Periegesis". Il caso singolare di Atene, «Acme», 54 (2001), 3-35: 21-23. 
logico e topografico di maggiori consensi $1{ }^{189}$ non si può fare a meno di notare una differenza sostanziale con la nostra quadriga: non si trattava di dediche ad Atena, quanto - con tutta probabilità - ai Dioscuri, venerati nell'Anakeion fuori dell'Acropoli. ${ }^{190}$ Una loro collocazione esterna ed anche la loro risistemazione ai tempi di Mnesicle avrebbe trovato sicuramente meno ostacoli di quella di una dedica ad Atena fuori del proprio recinto sacro, costituito dall'Acropoli. ${ }^{191} \mathrm{Ma}$ ormai la temporanea posizione esterna della quadriga si era trasformata in una sorta di communis opinio.

\subsection{3) NUOVE PROPOSTE DI COLLOCAZIONE PER LA QUADRIGA ORIGINALE E PER IL SUO RIFACIMENTO; IL POSSIBILE RAPPORTO CON IL MONUMENTO DI AGRIPPA: LOLLING, STUDNICZKA}

Partendo quindi dal presupposto di dover cercare, oltre a una localizzazione interna per l'originale, una esterna per la quadriga rinnovata, Lolling avanzava la candidatura di due siti in particolare. Per quanto riguarda la dedica originale Lolling era il primo (l'intuizione sarà sviluppata e in un secondo tempo corretta da Judeich) ${ }^{192}$ a proporne l'identificazione con i resti della base quadrata ${ }^{193}(5,5 \times 5,5 \mathrm{~m})$ attribuita alla Promachos stessa negli studi precedenti e poi di nuovo negli studi successivi (Lolling considerava le misure della base quadrata insufficienti per la Promachos, ma adatte alla quadriga; per la Promachos proponeva la base vicina, secondo lui di 7,1 $18,2 \mathrm{~m}){ }^{194} \mathrm{La}$ copia di $\mathrm{V}$ secolo avrebbe

189 La ricostruzione è quella di STEVENS, Architectural Studies Concerning the Acropolis of Athens, «Hesperia», 15 (1946), 73-106: 82-83, precisata nell'iconografia da L. BESCHI, Contributi di topografia ateniese, «ASAA», 29-30 (1967-1968), 511-36: 528-31.

190 Un tempo ubicato dagli studiosi sulle pendici settentrionali dell'Acropoli, ora, in conseguenza delle scoperte di G.S. DonTAS, The True Aglaurion, «Hesperia», 52 (1983), 48-63 relative all'Aglaurion che all'Anakeion doveva essere vicino (Paus. I 18, 2 3), viene posto quasi unanimemente su quelle orientali: cfr. M. SAPORITI, Il santuario di Aglauro, in GreCO, Topografia di Atene, 159 (contra BESCHI, Atene, 499).

191 V. a proposito della quadriga par. 3.2.4, 3.2.11, 4.

192 V. par. 3.2.9.

193 H.G. Lolling, Hellenische Landeskunde und Topographie, in F. HommeL J. Jung - H.G. LOLLING - B. Niese -R. PÖHLMANn - O. Richter, Geographie und politische Geschichte des klassischen Altertums, Nördlingen 1889, 343 n. 2.

194 LOLLING, Hellenische Landeskunde, 352 n. 1. 
potuto trovare spazio, seguendo le indicazioni di Erodoto, ove in seguito sarebbe sorto il Monumento ad Agrippa: ma allora Pausania ne avrebbe dovuto parlare all'ingresso; scartata poi anche l'idea di una posizione defilata a Nord del Monumento prima di scendere le scale per la fonte Clessidra (proposta definita dallo stesso Lolling "nicht wahrscheinlich": bisognerebbe sempre spiegare perché Pausania non l'avrebbe nominata scendendo proprio da quella parte), ${ }^{195}$ rimaneva per Lolling un'unica possibilità teoricamente valida sia per Erodoto sia per Pausania: l'atrio nordorientale dei Propilei, pur con qualche titubanza riguardo al valore di

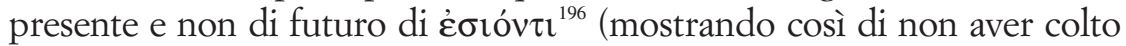
gli esiti del dibattito linguistico precedente, che aveva evidenziato come unico valore inusuale proprio quello di futuro! I problemi sollevati da questa interpretazione, infatti, non sono grammaticali, ma di spazio). ${ }^{197}$ Per meglio riconciliare le due testimonianze Lolling elaborava comunque un'ulteriore teoria: per il monumento di Agrippa sarebbe stata riutilizzata la quadriga per la vittoria sui Beoti e sui Calcidesi, vista quindi da Pausania esattamente dove la vedeva Erodoto, ma raggruppata poi con la Promachos nella descrizione. ${ }^{198}$ Particolarmente d'accordo si sarebbe mostrato in seguito F. Studniczka, il quale sosteneva che Pausania avesse ignorato il riuso del monumento come nel caso delle statue dei cavalieri all'ingresso dell'Acropoli. ${ }^{199}$

\subsection{4) PER UNA COLLOCAZIONE INTERNA ALL'ACROPOLI DELL'ORIGINALE E DEL RIFACIMENTO: IL FATTORE RELIGIOSO; IL LUOGO DI RITROVAMENTO DELLE ISCRIZIONI: FURTWÄNGLER}

Tuttavia le statue dei cavalieri venivano comunque descritte seguendo l'ordine topografico, e già alla ricostruzione di Lolling obiettava Furtwängler che l'intero basamento di Agrippa è assai recente ("das Postament gehört, wie seine Technik zeigt, von Grund auf römi-

195 Paus. I 28, 4.

196 LolLING, Hellenische Landeskunde, 343 n. 2.

197 V. n. 130.

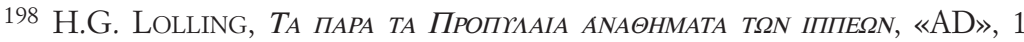
(1889), 179-99: 194 n. 2.

199 F. STUDNICZKA, Kalamis: ein Beitrag zur griechischen Kunstgeschichte, Leipzig 1907, 62. 
scher Zeit an": ${ }^{200}$ W.B. Dinsmoor ne ha poi precisato la datazione all'età pergamena, regno di Eumene II). ${ }^{201}$ Più in generale lo studioso si mostrava scettico su collocazioni esterne all'Acropoli e spostamenti pochi anni dopo il rifacimento: ${ }^{202}$ oltre al già ricordato uso linguistico di Erodoto, che a rigore farebbe pensare ad una collocazione addirittura interna ai Propilei difficilmente accettabile per motivi di spazio - sia che si intendano i Nuovi sia che si intendano gli Antichi, poco chiaro sarebbe risultato anche Pausania. Ma decisivo era per Furtwängler il fattore religioso: perché gli Ateniesi avrebbero dovuto consacrare una dedica ad Atena fuori dell'Acropoli, cioè all'esterno del temenos della divinità? ${ }^{203} \mathrm{~A}$ ciò aggiungeva quello che può essere considerato soltanto un indizio: il luogo di ritrovamento di entrambe le iscrizioni è interno all'Acropoli. Se quest'ultima considerazione può naturalmente essere ridimensionata da quanto sappiamo del riuso delle iscrizioni, l'attenzione al dato religioso illumina un aspetto fino allora accennato soltanto da P. Weizsäcker ${ }^{204} \mathrm{e}$ che soprattutto nell'antichità aveva un peso non indifferente. I successivi studi, tuttavia, generalmente non ne tengono conto. ${ }^{205}$

\subsection{5) IL PASSO ERODOTEO E UNA SUA POSSIBILE TRADUZIONE; ANCORA DEGLI ANTICHI PROPILEI E DELLO SPOSTAMENTO DELLA QUADRIGA: MILLER}

Maggiore risonanza ebbe infatti nello stesso anno il saggio di W. Miller, più specificamente dedicato all'Acropoli. Sicuramente a ragione egli ritornava sulla traduzione ormai consueta del passo di Erodoto, proponendo, invece di "It stands on the left just as you enter the Akropolis through the Propylaia", ${ }^{206}$ "the first thing you see on the left as you enter

200 FURTWÄNGLER, Meisterwerke, 14 n. 8; replica (più convinta che convincente) in F. STUDNICZKA, Archäologisches aus Griechenland, «AA», 36 (1921), 308-40: 317. 201 W. B. Dinsmoor, The Monument of Agrippa at Athens, «AJA», 24 (1920), 83; cfr. HolTZMAnN, L'acropole, 216; MONACO, Il monumento di Eumene II/Agrippa, in GRECO, Topografia di Atene, 78-80; sul monumento nel suo complesso v. KORRES, ANAQHMATIKA, 314-19.

202 Soprattutto se si ipotizzava il 446 a.C. come data del ripristino: ma su questo v. BERTI, La dedica degli Ateniesi, 19-40.

203 FURTWÄNGLER, Meisterwerke, 14.

204 WeIzSÄCKen, Pausanias und die Bildwerke, 6.

205 Per le eccezioni v. par. 3.2.11, 3.2.18.

206 Corrispondente al "gleich beim Eintritt" di STEIN, Jahresbericht über Herodot für 1879, 93-94. 
the Propylaia is the bronze chariot": 207 sulla scelta dei tempi, come si è visto, si può discutere ${ }^{208}$ ma non si può dire che l'attribuzione a $\pi \rho \tilde{\omega} \tau o v$ di un valore predicativo anziché avverbiale sia grammaticalmente scorretta. ${ }^{209}$ Tale proposta di traduzione assume ovviamente un significato topografico ed andrà quindi tenuta presente. ${ }^{210}$ Tuttavia Miller preferisce soffermarsi su altre due argomentazioni: informato di una parte del dibattito a lui precedente, esclude sulla base dell'uso linguistico erodoteo e dell'ordine topografico di Pausania una collocazione della quadriga esterna ai Propilei, per giungere, sebbene inconsapevolmente, alla medesima conclusione di Stein: ${ }^{211}$ Erodoto si riferirebbe qui agli Antichi Propilei, al cui interno si sarebbe trovata la quadriga; esattamente come Stein, Miller sottolineava che i Propilei andavano intesi nei confronti dell'Acropoli come un vestibolo e non come un semplice cancello, cosicché "entrando" o "entrati nei Propilei" non poteva significare un ambiente situato oltre (cioè l'Acropoli), bensì l'interno dei Propilei premnesiclei. Dunque "the chariot must have stood in the old Propylaia, or just in front of it. In it there was an abundance of room even for this colossal monument". Sull'abbondanza di spazio è lecito però nutrire qualche dubbio: ${ }^{212}$ anche se si trattava di una costruzione meno articolata dei Nuovi Propilei, il passaggio doveva essere garantito; inoltre il senso di innalzare la dedica all'interno di un edificio sarebbe stato per L. Weber assai scarso. ${ }^{213}$ Coll'alternativa poi di una collocazione della quadriga dinanzi ai Propilei Miller ritornerebbe a Curtius, ${ }^{214}$ già confutato, oltre che da Wachsmuth, ${ }^{215}$ da Stein. ${ }^{216}$ In effetti qualche fraintendimento o mancanza di informazioni devono essere all'origine di alcune strane affermazioni, come per esempio che "the inscriptions we possess are certainly considerably later than $507 ",{ }^{217}$ quando nessuno studioso ha mai dubitato che la forma delle let-

207 Miller, A History of the Akropolis, 504.

208 V. par. 3.1.10, 3.1.14, 3.1.15.

209 Contra WeBER, LYKA 'EФ 'EPMHI, 96.

210 V. par. 4.

211 STEIN, Jahresbericht über Herodot für 1879, 95.

212 Scettico FURTWÄNGLER, Meisterwerke, 14; cfr. anche n. 124. Lidea è comunque stata nuovamente ripresa: v. par. 3.2.19.

213 WEBER, ГYKA 'ЕФ 'ЕРMHI, 86-87.

214 CuRTIUs, Zur Topographie, 53-54, v. par. 3.1.10.

215 WaCHSMUTH, Der Standort des ehernen Viergespann, 20-23.

216 STEIN, Jahresbericht über Herodot für 1879, 93-94.

217 MiLLER, A History of the Akropolis, 507. 
tere dell'epigrafe più antica potesse farla risalire alla fine del VI secolo. ${ }^{218}$ Eppure questa considerazione serviva a Miller per avanzare una sua ipotesi molto più complessa, che prevedeva, nella sua troppo ottimistica ricapitolazione finale ${ }^{219}$ 1) la dedica della quadriga nei pressi dei ceppi e del Tempio arcaico di Atena, in corrispondenza di fondazioni vicino alla base della Promachos ${ }^{220} 2$ ) un primo ripristino (o addirittura la prima dedicazione, come sosteneva Kirchhoff prima del 1887!) del monumento negli o di fronte agli Antichi Propilei dopo le Guerre Persiane, periodo al quale risalirebbe la più antica delle iscrizioni che abbiamo (in disaccordo con la paleografia di VI secolo!), 3) lo spostamento della dedica all'interno dell'Acropoli dove l'avrebbe vista Pausania. Si diffondeva così nuovamente l'idea che la quadriga avesse cambiato posizione nel tempo. Nonostante alcune incongruenze, Miller va comunque ricordato non solo per aver proposto una diversa traduzione dell'espressione erodotea, ma anche per aver considerato seriamente l'idea che Erodoto potesse parlare degli Antichi Propilei.

\subsection{6) LO SPOSTAMENTO DELLA QUADRIGA E LE CONOSCENZE DI ERODOTO: HAUVETTE}

In questo abbastanza simile era la posizione di A. Hauvette, il quale però conosceva e citava espressamente le idee di Stein in merito. ${ }^{221}$ Hauvette analizzava comunque il problema a partire da una prospettiva originale, prendendo innanzitutto in esame la frase aĭ $\pi \varepsilon \rho$ हैं $\tau$

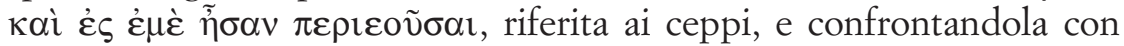

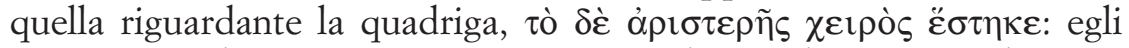
notava come la prima potesse spiegarsi molto meglio se scritta lontano

218 V. IG, I. $2^{3}$, ed. LEWIS - JeFFERY adiuv. E. ERXLEBEN, 489 n 501, con confronti paleografici (IG I3 627-29 e 646-55).

219 Miller, A History of the Akropolis, 508: "thus we have established several things beyond fear of successful contradiction".

${ }^{220}$ Miller, A History of the Akropolis, 507 e tav. XV. Lidentificazione dovrebbe coincidere con quelle di Lolling (v. par. 3.2.3) e di Judeich (nella prima edizione: v. par. 3.2.9) per la Promachos, poi di Judeich (nella seconda edizione: v. par. 3.2.9) per la quadriga, ma le misure date da Miller sono molto diverse: 2,5-3 m, apparentemente incompatibili anche con la rappresentazione alla tav. XV, ove il lato più corto ha dimensioni prossime a quelle della base della Promachos.

221 Hauvette citava la quinta edizione del commento a Erodoto (Herodotos erkl. H. STEIN, I.1, Berlin $1883^{5}$, XXII n. 1). 
da Atene ("c'erano ancora ai miei tempi"), ${ }^{222}$ mentre la seconda avrebbe potuto essere un'aggiunta ateniese come sosteneva Bauer, ${ }^{223}$ ma soltanto in teoria, visto che è al contrario perfettamente integrata nel resto del capitolo, grazie a una serie di rimandi interni. ${ }^{224}$ Dunque il tutto sarebbe stato scritto lontano da Atene, ma non per questo Erodoto avrebbe scritto per sentito dire: ${ }^{225}$ egli riporta infatti i versi precisamente nell'ordine conservato dall'iscrizione rinnovata, ciò che escluderebbe una derivazione "libresca" da una raccolta di epigrammi. ${ }^{226} \mathrm{Ma}$ allora la situazione che Erodoto aveva osservato personalmente e poi descritto sarebbe anteriore ai lavori dell'Acropoli. ${ }^{227}$ Dunque anche per Hauvette la quadriga cambiò posizione: inizialmente "le quadrige et les chaînes formaient une sorte de groupe adossé à la muraille de l'Acropole"; in seguito essa sarebbe stata ripristinata nello spazio antistante la porta dell'Acropoli, come sosteneva Stein con assai scarse giustificazioni, ${ }^{228}$ infine sarebbe stata trasferita, a causa dei lavori di Mnesicle, all'interno dell'Acropoli, dove l'avrebbe vista Pausania; Erodoto non avrebbe saputo dello spostamento o in alternativa avrebbe evitato di registrarlo. ${ }^{229}$

\subsection{7) SCETTICISMO SULLA POSSIBILITA' DI UNA SOLUZIONE E/O NUOVE DISCUSSIONI SULLA BIOGRAFIA DI ERODOTO: FRAZER, WACHSMUTH, KÖRTE, SCHMOLLING, POWELL, MYRES}

Di Hauvette come di altra bibliografia precedente cercava di tenere conto Frazer, ma senza arrivare a soluzioni univoche, bensì elencandole tutte in una ricostruzione un po' contraddittoria: ${ }^{230}$ colpisce però

${ }^{222}$ Ma su questo v. par. 3.2.7.

223 BAuer, Themistokles, 16 n. 1; v. par. 3.1.17. Così già WeIzsÄCKer, Die Aufstellung der Bildwerke, 110; v. par. 3.1.6.

224 A. Hauvette, Herodote historien des guerres mediques, Paris 1894, 48-49.

225 Così per es. BACHOF, Zu Herodotos, 177-82; v. par. 3.1.17.

226 Vedi però ultimamente H. ERBSE, $\mathrm{Zu}$ den Epigrammen des Simonides, «RhM», 141 (1998), 213-30: 224-25; su questo cfr. par. 3.2.17.

227 HauvetTe, Herodote, 52.

228 STEIN, Jahresbericht über Herodot für 1879, 95; v. par. 3.1.16.

229 HauvetTe, Herodote, 50-52.

230 Pausanias's Description of Greece transl. with a comm. by J.G. FRAZER, London-New York 1898, II, 352-53; V, 514. 
che, nonostante la sua conclusione tra il rassegnato e l'ironico ("the question of the exact position of the chariot seems, as our knowledge stands at present, incapable of a definitive solution. It has greatly exercised the archeologists"), egli cercasse di sostenere la vecchia ipotesi di una collocazione interna ai Propilei con l'idea smentita dai fatti di una quadriga di dimensioni limitate..$^{231}$

A questo punto una certa sfiducia nei termini della questione sembra coinvolgere anche uno dei protagonisti. Wachsmuth in una serie di interventi decide di associarsi alla posizione per così dire "antierodotea" già di Bachof: la quadriga si sarebbe trovata all'interno dell'Acropoli, vicino alla Promachos, dove la vedeva Pausania; semplicemente Erodoto, non più tornato ad Atene dopo la partenza per Turi, avrebbe male interpretato il racconto di un suo corrispondente. ${ }^{232}$ Veniva così meno il desiderio di correggere il testo. Non si esauriva però la discussione intorno alla biografia di Erodoto. ${ }^{233}$

Proprio a partire da questo passo, infatti, Körte deduce che Erodoto si sarebbe trovato a Turi e avrebbe usato l'imperfetto per descrivere la posizione dei ceppi perché si aspettava che le mura annerite dal fuoco persiano sarebbero state presto sostituite nel corso dei restauri dell'Acropoli, ma non avrebbe pensato alla riorganizzazione dello spazio dovuta ai Propilei di Mnesicle, utilizzando quindi un perfetto resultativo a proposito della quadriga. ${ }^{234}$

Anche per E. Schmolling Erodoto avrebbe rielaborato questa parte delle Storie a Turi, descrivendo però una situazione dell'Acropoli precedente alla sua partenza: diversamente Erodoto avrebbe scritto che

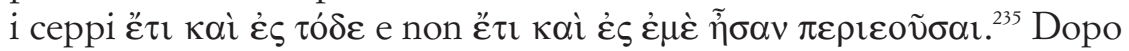

231 Pausanias's Description, II, 353. Contra, in base ai frammenti ritrovati, Weller, The Pre-Periclean Propylon, 64-65 n. 1; WeBER, гrKA 'EФ EPMHI, 98.

232 C. WACHSMUTH, Einleitung in das Studium der alten Geschichten, Leipzig 1895, 512 n. 1; ID., Bemerkungen zu griechischen Historikern, «RhM», 56 (1901), 215. 26: 215; ID., Athenai, 203-04.

233 Uno dei primi a reagire è E. MEYER, Forschungen zur alten Geschichte, II, Halle 1899, 196.

234 G. KÖRTE, recensione a E. PETERSEN, Die Burgtempel der Athenaia, Berlin 1907, «GGA», 170, II (1908), 837-52: 845 n. 1.

235 E. SCHMOLLING, Herodots Berichte über Athen im Lichte der neueren Funde auf der Akropolis, «Sokrates», 1 (1913), 687-704: 693 n. 1 mediante il confronto con Her. VII 123. 
queste eccessive sottigliezze, con cui quasi si pretenderebbe di insegnare a scrivere a Erodoto, Schmolling spiegava invece che il perfetto

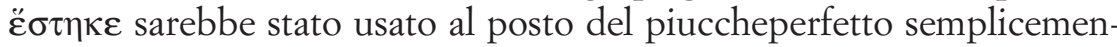
te per non dare un'impressione di pedanteria.

Proprio sul cambiamento dei tempi verbali tra la descrizione dei ceppi e quella della quadriga si soffermava invece J.E. Powell, senza poter però concludere se Erodoto avesse ricavato l'informazione da qualcuno o per autopsia, né se i Propilei, visti o no personalmente, fossero quelli di Mnesicle o l'ingresso precedente e, di conseguenza, neppure se Erodoto scrivesse questo passo ad Atene nel 437 o dopo $;^{236}$ con maggiore decisione e riprendendo inconsapevolmente le tesi di Bauer ${ }^{237}$ e, ancor prima, di Weizsäcker, ${ }^{238}$ J.L. Myres arguiva, dalla diversità dei tempi verbali e dalla convinzione che le mura annerite dal fuoco non sarebbero dovute rimanere tali (questo perché Myres pensava a mura di cinta), ${ }^{239}$ "the certainty that Herodotus described the remodelled monument as well as remembered the original" ${ }^{240}$

A mio parere il rischio di interpretazioni simili è quello di forzare il testo di Erodoto. Le espressioni del tipo "sino ai miei tempi" 241 potrebbero forse anche significare la lontananza di Erodoto dai luoghi descritti, ma non possono fornirci con sicurezza questo genere di informazioni biografiche; per G. Nenci le espressioni vanno prese alla lettera: con esse Erodoto semplicemente indica il proprio tempo. ${ }^{242}$ Credo anzi che si tratti di un modo del tutto naturale per Erodoto di mettersi nella prospettiva del lettore o più in generale del suo pubblico futuro, quasi fosse il destinatario di una lettera.

236 J.E. Powell, The History of Herodotus, Cambridge 1939, 69. Per la data di composizione delle Storie v. ora da un lato J. COBET, Wann würde Herodots Darstellung der Perserkriege publiziert?, «Hermes», 105 (1977), 2-27 (fra 430 e 426 a.C.) e J.A.S Evans, Herodotus' Publication Date, «Athenaeum», n.s. 57 (1979), 145-49 (poco dopo il 424 a.C.) entrambi con bibliografia per gli studi precedenti; dall'altro C.W. FORNARA, Evidence for the Date of Herodotus' Publication, «JHS», 91 (1971), 25-34 (poco prima del 414 a.C.), sempre con bibliografia.

237 BAUER, Themistokles, 16 n. 1; v. par. 3.1.17.

238 WeIZsäCKER, Die Aufstellung der Bildwerke, 110; v. par. 3.1.6.

239 Ma v. par. 3.2.12 per la discussione su dove fossero appesi i ceppi.

240 J.L. MYRES, Herodotus Father of History, Oxford 1953, 10-11.

241 In parte già raccolte e discusse da BACHOF, $Z$ u Herodotos, 177, sono catalogate da J.E. Powell, A Lexicon to Herodotus, Cambridge 1938, 150 s.v. ह̌ı

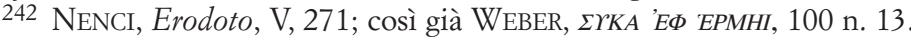




\subsection{8) LE INDAGINI ARCHEOLOGICHE: IL TAGLIO NELLA ROCCIA PER LA COLLOCAZIONE DELLA QUADRIGA RIFATTA ALL'ESTERNO DELL'ACROPOLI: WELLER}

Dopo tanto dibattere sulle fonti, la svolta sembrò giungere dal campo archeologico: C.H. Weller, nel corso della sua indagine centrata sugli Antichi Propilei, ritenne di aver individuato dei tagli nella roccia che avrebbero potuto accogliere la base della quadriga. ${ }^{243}$ Essi si trovano attualmente di fronte ai Propilei, sulla destra, ma rispetto al Propylon diversamente orientato che Erodoto poteva aver visto erano proprio a sinistra, anche rispetto a sei gradini, "remains of some early ascent", ${ }^{244}$ ciò che implicherebbe naturalmente la ripresa della diffusa ma discutibile traduzione di Her. V 77 come "a sinistra per chi è in procinto di entrare". Lo stesso Weller si mostrava abbastanza cauto nell'identificazione: "they are only partially exposed, so that it is impossible to learn with exactness the kind of foundation for which they are designed", concludendo pero fiduciosamente che "at least there is nothing inconsonant with the theory that the chariot stood upon them, or at least in close connection with them". ${ }^{245}$ A dire il vero i rilievi condotti in situ da R. Bohn evidenziavano misure insufficienti per le dimensioni della quadriga (lato iscritto di almeno $3 \mathrm{~m}$ ): il taglio cui si riferisce Weller avrebbe infatti una larghezza compresa tra 2,30 e 2,35 $\mathrm{m}$ e una lunghezza di 2,05 $\mathrm{m}^{246}$ laddove proprio Weller per primo ha calcolato, a partire dai resti della base iscritta e mediante la misurazione delle lettere, le dimensioni complessive dell'iscrizione in $2,84 \mathrm{~m}$, ai quali aggiungere eventuali margini. ${ }^{247} \mathrm{Ma}$ Weller era convinto della bontà

243 Weller, The Pre-Periclean Propylon, tav. I lettera K.

244 Weller, The Pre-Periclean Propylon, 69-70 + tav. I. Su questa rampa molto più dubbioso era R. BoHn, Die Propylaeen der Akropolis zu Athen, Berlin-Stuttgart 1882, 17, che lasciava aperte varie possibilità per l'interpretazione dei resti che aveva già individuato come "stufenartig".

245 Weller, The Pre-Periclean Propylon, 64.

246 R. Bohn, Die Propylaeen, tav. XV n. 9.

247 Weller, The Pre-Periclean Propylon, 64 n. 1, che aggiorna le dimensioni (2,30 $\mathrm{m}$ di iscrizione; 2,50-3,00 m di lato iscritto) calcolate da MiCHAELIS, Bemerkungen zur Periegese, 96, ritenute sottostimate in quanto ricavate dal fac-simile dell'iscrizione. Per errore vengono riportati $6,00 \mathrm{~m}$ da parte di SCHOLLMEYeR, Antike Gespanndenkmäler, 58 n. 39, ripreso da KLuwe, Das Siegesmal, 274. 
della teoria dello spostamento nella versione delineata da Hauvette, ${ }^{248}$ dunque considerando ottimisticamente "the location of the chariot (...) established on literary evidence" ${ }^{249}$ diventava naturale interpretare di conseguenza i tagli nella roccia evidenziati da Bohn, ${ }^{250}$ tenuto conto anche delle critiche mosse a quest'ultimo a proposito delle sue misurazioni. ${ }^{251}$ Non bisognerà però dimenticare l'origine in realtà letteraria della proposta di identificazione archeologica di Weller, che parte dal presupposto, contestato, come si è visto, dal punto di vista linguistico e religioso, di una temporanea collocazione all'esterno dell'Acropoli.

\subsection{9) LA COLLOCAZIONE INTERNA ALL'ACROPOLI DELLA QUADRIGA ORIGINALE, LA COLLOCAZIONE ESTERNA ALL'ACROPOLI DELLA QUADRIGA RIFATTA, LO SPOSTAMENTO ALL'INTERNO DELL'ACROPOLI: JUDEICH}

All'affermazione della teoria dà un contributo fondamentale W. Judeich in due successive edizioni della sezione dedicata alla topografia ateniese dall'autorevole Handbuch der klassischen Altertumswissenschaft dell'editore Beck di Monaco. ${ }^{252}$ Judeich infatti, aderendo alla teoria dello spostamento, non era tenuto a cercare per la quadriga una collocazione nella parte sinistra dell'Acropoli, in quanto Erodoto descriverebbe la quadriga nella fase in cui si trovava a sinistra dinanzi ai Propilei premnesiclei: Judeich, come già anticipato da Lolling, ${ }^{253}$ poteva quindi prendere in considerazione tra l'Eretteo ed i Propilei, in accordo con l'itinerario di Pausania (I 28, 2), due basi vicine, rispettivamente di 5,5x5,5 m e di 5x8,5 $\mathrm{m}$, la prima praticamente in asse con i Propilei di Mnesicle, la seconda (meno riconoscibile) spostata più a destra, negli studi precedenti alternativamente indicate come basamento della Promachos; vista la menzione ravvicinata da parte di Pausania, era possibile che l'altra base fosse quella della nostra quadriga. Quale delle due? Anche sulla posizione della Promachos si è effettivamente dibattuto, tanto che Judeich in un primo

248 HauvetTe, Herodote, 50-52; v. par. 3.2.6.

249 Weller, The Pre-Periclean Propylon, 64.

250 BoHn, Die Propylaeen, 17: egli si limitava a osservare il diverso orientamento rispetto ai Propilei e quindi la precedenza cronologica.

251 Weller, The Pre-Periclean Propylon, 36.

252 W. JudEICH, Topographie von Athen, München 1905 e München $1931^{2}$.

253 LOLLING, Hellenische Landeskunde, 343 n. 2. V. par. 3.2.3. 
momento le attribuiva la base più a destra in ragione delle maggiori dimensioni, ${ }^{254}$ in seguito tornava all'identificazione con la base quadrata perché in asse con i Propilei, ${ }^{255}$ di conseguenza la quadriga otteneva dagli studiosi prima la base centrale di $5,5 \times 5,5 \mathrm{~m}$, poi definitivamente la base sulla destra di $5 \times 8,5 \mathrm{~m}$, misure a dir poco più che abbondanti per le sue necessità. Comunque quest'ultima - o meglio le sue vicinanze, in quanto successivamente letta da Stevens come piattaforma terrazzata che introduceva alla Promachos ${ }^{256}$ - è grosso modo diventata la localizzazione ufficiale della quadriga, almeno nella fase in cui si doveva trovare all'interno dell'Acropoli: peraltro non ci si è più posti il problema di cercarla "a sinistra per chi entra nei Propilei”, in virtù dell'ipotesi dello spostamento. Così per Judeich la quadriga sarebbe stata originariamente consacrata di fronte all'Antico Tempio di Atena (tracce di una base di 5x8,5 m), sarebbe stata poi ripristinata dinanzi ai Propilei (taglio di 2,35x2,05 m) per evitare l'eccessiva vicinanza con la Promachos - lo studioso è il primo a ipotizzare questa motivazione per così dire "estetica" 257 - infine sarebbe ritornata nella collocazione originale per cause di forza maggiore: meglio la vicinanza con la Promachos che il danneggiamento in seguito alla costruzione dei Nuovi Propilei. ${ }^{258}$ Dopo aver messo in contraddizione Erodoto e Pausania si poteva così superare il contrasto attribuendo loro la descrizione di due diverse fasi. Non si notava però un'altra possibile contraddizione: in questo modo due basi di eguali dimensioni avrebbero dovuto occupare tagli di dimensioni così diverse. Ciononostante la teoria dello spostamento come sistematizzata da Judeich e con le due localizzazioni proposte si avviava a diventare una sorta di vulgata (successivamente Judeich, rimanendo fermo nelle sue posizioni, si sarebbe limitato a richiamare, come già Curtius, ${ }^{259}$ la vicenda dei cavalieri dedicati sulle pendici

254 JudEICH, Topographie von Athen, München 1905, 215-16 con n. 8 e bibliografia (in disaccordo però con la tav. II: die Akropolis und ibre Abbänge).

255 JudeIch, Topographie von Athen, München $1931^{2}, 234-35$ con n. 1 e bibliografia.

256 Stevens, The Periclean Entrance Court, 509-10.

257 Poco convinto della spiegazione appariva già M.L. D'Ooge, The Acropolis of Athens, New York 1908, 303: "if the close proximity of the statue of Athena Promachos was felt to be an objection to the old site at the first, how could this objection become less keenly felt at a later time?"

258 JudEICH, Topographie von Athen, München 1905, 217 n. 8.

259 CurTIUs, Die Stadtgeschichte, 155; v. par. 3.2.2. 
dell'Acropoli; ${ }^{260}$ ma della non sovrapponibilità della vicenda in quanto non si trattava di offerte ad Atena si è già detto). ${ }^{261}$

\subsubsection{0) LA QUADRIGA ORIGINALE E IL SUO RIFACIMENTO: ENTRAMBI ALL'INTERNO DELL'ACROPOLI O ENTRAMBI ALL'ESTERNO (CON O SENZA SPOSTAMENTO FINALE ALL'INTERNO)? FURTW ÄNGLER, KÖRTE, ROBERT}

Le reazioni alla sistematizzazione di Judeich furono all'inizio variegate: se da un lato Furtwängler, per nulla convinto dell'opportunità dello spostamento, restava favorevole ad una continuità all'interno dell'Acropoli, ${ }^{262} \mathrm{G}$. Körte sosteneva, all'opposto, la presenza della quadriga all'esterno non in una, ma addirittura in due fasi della sua esisten$\mathrm{za}^{263}$ la quadriga si sarebbe trovata anche in origine dinanzi ai Propilei, eliminando quindi sin dall'inizio il collegamento con i ceppi, che pure erano stati dedicati per gli stessi avvenimenti. Körte era poi così sicuro che l'espressione di Erodoto (V 77), nonostante tutti i dubbi avanzati dalla riflessione linguistica precedente, significasse "vom Eingang", da utilizzarla come prova che Erodoto si riferisse agli Antichi Propilei, in quanto davanti a quelli di Mnesicle non sarebbe bastato lo spazio. A parte queste ipotesi costruite su altre ipotesi - oltretutto contestate - si è già avuto modo di vedere come l'indicazione data da Erodoto assumesse per Körte un valore altresì cronologico e biografico. ${ }^{264}$

La localizzazione esterna della quadriga proposta da Weller ${ }^{265}(\mathrm{e}$ ripresa da Judeich ${ }^{266}$ trova entusiastica accoglienza anche presso C. Robert: egli, a partire da quell'ipotesi e dopo aver postulato l'inaffidabilità topografica di Pausania, deduceva che la quadriga non si fosse

260 JuDEICH, Topographie von Athen, München $1931^{2}, 239$.

261 V. par. 3.2.2.

262 A. FURTWÄNGLER, Zu den Skulpturen der archaischen Bauten der Akropolis zu Athen, «SBAW», 1906, 143-150: 147-48: qui Furtwängler criticava in particolare il brevissimo intervallo di tempo intercorso tra la ridedicazione e lo spostamento (446-437 a.C., nell'ipotesi allora più diffusa di un ripristino dopo la rivolta dell'Eubea; un po' diverso con l'ipotesi di Enofita, per lo meno una generazione: 457-37 a.C.).

263 KÖRTE, recensione a E. PETERSEN, 844-45 n. 1.

264 KÖRTE, recensione a E. PETERSEN, 845 n. 1; v. par. 3.2.7.

265 Weller, The Pre-Periclean Propylon, tav. I, lettera K.

266 JudEICH, Topographie, München 1905, 198 fig. 22. 
mai mossa dallo spazio antistante i Propilei, dove l'avrebbe vista Erodoto ${ }^{267} \mathrm{Ma}$ in realtà non è possibile squalificare in questo modo una fonte antica sulla base di una identificazione ipotetica e dell'ulteriore ipotesi della permanenza esterna del monumento. La spiegazione poi che Pausania si distaccasse sensibilmente dall'ordine topografico per motivi stilistici, vale a dire per ottenere qui $(\mathrm{I} 28,2)$ una conclusione ad effetto, ${ }^{268}$ non trova riscontro, visto che di conclusione non si tratta: ${ }^{269}$ segue infatti la menzione delle statue di Pericle e dell'Atena Lemnia, delle mura, infine dell'uscita (I 28, 2-3); è peraltro nota l'abitudine di Pausania di segnalare un suo eccezionale scostamento dall'ordine topografico. $^{270}$

\subsubsection{1) LA COLLOCAZIONE DELLA QUADRIGA, ORIGINALE E RIFATTA, ALL'INTERNO DELL'ACROPOLI; ANCORA DEL FATTORE RELIGIOSO: WEBER}

L'artificiosità della contrapposizione fra Erodoto e Pausania dovrebbe a questo punto essere chiara; ciò fu evidenziato con particolare forza da L. Weber, che si poneva tra l'altro in un'ottica totalmente diversa: per lui la nuova dedica si presentava soprattutto come un atto di pietà nei confronti della divinità poliade e verso gli antenati. ${ }^{271}$ Ecco perché gli Ateniesi non avrebbero scelto un posto diverso da quello individuato in precedenza, giudicandolo sacro e provando un rispetto reverenziale per le imprese dei loro avi. ${ }^{272} \mathrm{Al}$ concetto generale si aggiungono semplici indizi, quali il già ricordato ritrovamento di entrambe le iscrizioni all'interno dell'Acropoli (ma sono noti gli spostamenti successivi cui potevano andare incontro le epigrafi, soprattutto se riutilizzate) e le uguali dimensioni delle basi, molto più facili da conservare nel caso di un ripristino nel medesimo luogo. ${ }^{273}$ L'idea, anzi,

267 C. Robert, Pausanias als Schriftsteller, Berlin 1909, 94.

268 ROBERT, Pausanias, 95.

269 WeBER, $\Sigma$ YKA ' $E \Phi$ 'EPMHI, 92, con ulteriori osservazioni stilistiche (90-93).

270 V. par. 3.1.11.

271 WEBER, ГYKA 'EФ EPMHI, 83.

272 WeBER, IYKA 'EФ 'EPMHI, 97.

273 WEBER, гrKA 'EФ EPMHI, 98; ID., Zum Viergespann auf der Akropolis, «PhW», 53 (1933), 331-36: 333. Va detto che i calcoli per la base più antica si fondano sull'improbabile divisione in sei blocchi; tuttavia che le basi fossero complessivamente di uguali dimensioni è comprovato dalla misura e dalla distribuzione delle lettere, cf. anche la ricostruzione e la descrizione di RAUBITSCHEK, Dedications, 191, 193, 201-02. 
il dogma dello spostamento ha portato in un vicolo cieco, ${ }^{274}$ in cui tutti hanno seguito Kirchhoff: ma Kirchhoff stesso lasciava indeterminato se la variazione nell'ordine dei versi fosse stata casuale o volesse segnalare la diversa posizione della quadriga! $!^{275}$

Non è infatti chiaro per quale motivo, se di fianco alla Promachos la quadriga rinnovata non trovava più collocazione efficace, la medesima quadriga potesse, dieci o meglio venti ${ }^{276}$ anni più tardi, tornare nella posizione iniziale. Weber a rigor di logica si chiedeva anche perché, riportata nel luogo originario la quadriga, il presumibilmente ristabilito collegamento spaziale tra le due parti dell'offerta votiva non avrebbe dovuto essere nuovamente espresso, ma fosse stato mantenuto l'ordine dei versi differente da quello dell'antico epigramma, che si sostiene cambiato a causa della collocazione dell'offerta rinnovata in un primo momento lontano dai ceppi. ${ }^{277}$ Qui però si pretenderebbe una nuova iscrizione soltanto per segnalare il cambiamento, laddove nell'ipotesi di Kirchhoff, dovendosi già restaurare la dedica, si sarebbe decisa la modifica, senza alcun carico di lavoro aggiuntivo né bisogno di altro materiale oltre a quello preventivato. ${ }^{278}$ Ciò che dell'argomentazione di Weber sembra avere più efficacia, rispetto ai sillogismi, è l'attenzione al dato religioso: ceppi e quadriga sono considerati parti complementari che formano il complesso dell'offerta, inseparabili spazialmente. Solo il rispetto per la dea è decisivo per la scelta del luogo e la realizzazione del dono. Le valutazioni di altro tipo (pratico, ma soprattutto estetico) peccano sempre di un certo modernismo: si fa l'esempio del fregio del Partenone, il quale, così ricco, non era destinato che alla dea e non al pubblico ${ }^{179}$ La pietas è quindi un elemento a favore del permanere della nuova quadriga nello stesso luogo. L'interpretazione, da parte di Weber, della trasposizione dei versi con i nomi dei nemici sconfitti all'inizio dell'epigramma come segno di un'epoca caratterizzata da una maggiore ostentazione ed elaborazione,

274 WEBER, ГrKA 'EФ EPMHI, 97.

275 KirChHOFF (Lolling), Bemerkungen zu dem Bruchstück, 114; v. par. 3.2.1.

276 Anche Weber seguiva l'ipotesi allora prevalente di un ripristino del monumento nel 446; tuttavia è meglio pensare al 457 o poco dopo, v. BERTI, La dedica degli Ateniesi, 19-40. Mnesicle mette mano ai Propilei nel 437.

277 WeBER, Zum Viergespann, 332.

278 KirchHoff (Lolling), Bemerkungen zu dem Bruchstück, 111-14; v. par. 3.2.1.

279 WeBER, Zum Viergespann, 333. 
appare invece quantomeno forzata, dato che non sappiamo neppure se la variazione fosse voluta. ${ }^{280}$

E' un fatto comunque che la nuova versione non esclude assolutamente la vicinanza dei ceppi, che costituivano una parte della dedica. ${ }^{281}$

\subsubsection{2) LA QUESTIONE DEI CEPPI}

Dove si trovavano dunque? Si era visto lo scarso consenso incontrato da Ross proponendo i blocchi del Propylon; ${ }^{282}$ un altro suggerimento presto lasciato cadere era giunto da Kirchhoff, che aveva parlato di una "Aufmauerung" che sarebbe stata costruita apposta di fronte al Tempio arcaico di Atena. ${ }^{283}$ Collocazione più naturale sono quindi sembrate le mura dell'Acropoli, ${ }^{284}$ in particolare quelle occidentali ${ }^{285}$ o quelle settentrionali. ${ }^{286}$

In seguito si è imposta però d'autorità la soluzione di W. Dörpfeld: i ceppi sarebbero stati appesi al muro di rinforzo del Tempio arcaico di Atena (che come sappiamo Dörpfeld chiamava Hekatompedon), vicino al quale sarà eretta anche la Promachos; il muro veniva a trovarsi in effetti "davanti al megaron volto ad Occidente" e la definizione erodotea (V 77, 3) di "mura bruciacchiate dal fuoco acceso dal Medo" ben si armonizzerebbe con la menzione all'incirca nello stesso luogo, da parte di Pausania (I 27, 6), delle "statue antiche di Atena... annerite e ... fragili... raggiunte dal fuoco quando gli Ateniesi s'imbarcarono sulle navi e il re prese la città" ${ }^{287}$

Per la verità Dinsmoor Jr. ha eccepito che il termine $\tau \varepsilon \tilde{\imath} \chi 0 \varsigma$ è normalmente usato non solo in Erodoto, ${ }^{288} \mathrm{ma}$ in genere in età classica, ${ }^{289}$

280 WeBER, гrKA 'EФ 'EPMHI, 106-08. A Weber sembrava strano che gli Ateniesi non conoscessero più il testo originale ai tempi del restauro, visto che un resto è giunto fino a noi, ma si può facilmente osservare che il frammento sarà stato sepolto fra le macerie della cosiddetta "colmata persiana" (o "Perserschutt").

281 WEBER, ГYKA 'EФ 'EPMHI, 97.

282 Ross, Archäologische Aufsätze, I, 78-81; v. par. 3.1.4 e 3.1.6.

283 KirchHoff (LOLLING), Bemerkungen zu dem Bruchstück, 113.

284 HauvetTe, Herodote, 50.

285 Per es. JudEICH, Topographie, München 1905, 217 n. 8.

286 Così aveva inizialmente pensato WEBER, IYKA 'EФ EPMHI, 108.

287 W. DöRPFELD, Das Hekatompedon in Athen, «JDAI», 34 (1919), 1-40: 12, 38-39.

288 POWELL, Lexicon, 352.

289 A Greek-English Lexicon, by LIDDELL - SCOTT, rev. and augm. by JONES, with MCKENZIE, 1767. 
soltanto con il significato di muro di cinta o fortificazione (o anche piazzaforte) ed ha quindi preferito pensare al lato interno del muro pelasgico a Nord del Propylon come luogo di esposizione dei ceppi, ${ }^{290}$ che tuttavia F. Jünger escluderebbe per motivi di spazio e opportunità. ${ }^{291}$

Tutto sommato, un muro di rinforzo sull'Acropoli a fondamenta di una sorta di terrazzamento poteva non essere considerato così diverso da una fortificazione: di certo concettualmente si avvicinava in maggior misura alle mura di una città piuttosto che ai muri di un edificio, per i quali sarebbe stato usato il termine $\tau$ õ $\chi 0 \varsigma$ (ciò che esclude i cosiddetti oikemata di una delle ipotesi di J. Hurwit). ${ }^{292}$ Il contesto religioso farebbe pensare ad una dedica più vicina al Tempio della divinità, come nel caso dei ceppi degli Spartani intorno al Tempio di Atena Alea a Tegea (Her. I 66, 4; Paus. VIII 47, 2), sebbene non si possano per questo escludere le mura esterne, per esempio proprio ad Atene in seguito utilizzate per il cosiddetto piccolo donario pergameno (Paus. I 25, 2) ${ }^{293}$ Potrebbe, d'altra parte, sembrare strano che Erodoto vedesse fino ai suoi tempi i ceppi su mura bruciate dai Persiani non ancora restaurate, se queste avevano scopo difensivo, anche se è vero che le prime ad essere ricostruite, e in gran fretta, furono le mura della città, ${ }^{294}$ più che le mura dell'Acropoli: sulla datazione di queste ultime la discussione è aperta, ${ }^{295} \mathrm{ma}$, anche superati i problemi cronologici, rimane difficoltoso sostenere che, una volta escluse per motivi di spazio le mura occidenta-

290 DinsmoOR JR., The Propylaia, I, 43 n. 25.

291 JÜNGER, Gespann und Herrschaft, 236-37, con riferimento alle costruzioni evidenziate da T. Tanoulas, The Pre-Mnesiclean Cistern on the Athenian Acropolis, «MDAI(A)», 107 (1992), 129-60: 152-60; almeno sulla costruzione della cisterna in età arcaica non si discute, cfr. HolTZMAnN, L'acropole, 72.

292 J.M. HuRWIT, The Athenian Acropolis, London 1999, 144; similmente SCHOLLMEYER, Antike Gespanndenkmäler, 57.

293 A. StewArt, Attalos, Athens, and the Akropolis. The Pergamene "Little Barbarians" and their Roman and Renaissance legacy, with an Essay on the Pedestals and the Akropolis South Wall by M. KORRES, Cambridge 2004, 181-98, con analisi tecnica e ricostruzione, a cura di Korres, dei basamenti delle statue presso, ma non sopra, il muro meridionale, 242-85, elenco completo delle fonti, 287-92 e bibliografia; per una collocazione direttamente sul parapetto v. B. ANDREAE, Laurea coronatur. Der Lorbeerkranz des Asklepios und die Attaliden von Pergamon, «MDAI(R)», 100 (1993), 83-106: 97-98 + tav. 16. Sul donario di Attalo v. ora L. MERCuri, Il donario di Attalo, in GrECO, Topografia di Atene, 118.

294 Thuc. I 89, 3; 90, 3; 93, 2.

295 Se Temistocle mirava soprattutto a cingere di mura la città, a lui pure 
li, ${ }^{296}$ candidate in astratto più naturali, le rimanenti mura settentrionali o meridionali si trovassero "davanti" o "di fronte" al megaron. In ogni caso, il muro di sostegno del Tempio arcaico di Atena, preferibile per motivi religiosi, poteva essere rimasto immutato, sebbene annerito dal fuoco, sino almeno alla costruzione dell'Eretteo, o anche oltre. La candidatura di questo muro è quindi ormai generalmente accettata, già a partire da punti di vista completamente divergenti fra loro quali quelli di Weber e di Judeich. ${ }^{297}$ Naturalmente vi è chi è tornato a metterla in dubbio, in base a diverse interpretazioni del termine megaron: $\mathrm{K}$. Jeppesen, ad esempio, ha preferito fare ricorso alla cella occidentale del Partenone anziché a quella del Tempio arcaico di Atena, con la conseguenza di collocare i ceppi sul muro di sud-ovest, di fronte al Partenone, in zona non obliterata dai restauri cimoniani, ed ha giustificato la lontananza tra quadriga e ceppi con le due successive e ravvicinate eppure distinte indicazioni topografiche date da Erodoto (V 77, 34) $;^{298}$ ad una ancora maggiore ed inaccettabile separazione dalla quadriga e dal suo contesto ${ }^{299}$ porterebbe la tesi di N. Robertson, il quale, cam-

solitamente si attribuisce l'iniziativa di ricostruire con materiale di recupero (ad esempio gli ancor oggi visibili rocchi di colonne) proprio il muro Nord dell'Acropoli attraverso il quale i Persiani avevano fatto irruzione; all'iniziativa di Cimone si è soliti poi attribuire il tratto relativo all'ingresso nell'Acropoli, con i Propilei pre-mnesiclei, mentre sicuramente cimoniano secondo le fonti sarebbe il muro meridionale (v. sinteticamente W. Johannowski, Atene, in Enciclopedia dell'arte antica, I, Roma 1958, 767 868: 790-93). Ad un medesimo periodo cimoniano sia per il muro Nord sia per il muro Sud sino a giungere all'età periclea pensa invece HuRWIT, The Athenian Acropolis, 142; 159-60, seguendo J.S. Boersma, Athenian Building Policy from 561/0 to 405/4 B.C., Groningen 1970, 46; 162, il quale però propendeva complessivamente per gli anni ' 60 ; il tratto nordoccidentale (con la possibile eccezione dei corsi inferiori) è datato da $\mathrm{T}$. TANOULAS, Structural Relations between the Propylaea and the NW Building of the Athenian Acropolis, «MDAI(A)», 107 (1992), 199-215: 210-215 alla riorganizzazione di Mnesicle; tuttavia per il muro settentrionale M. KORRES, On the North Acropolis Wall, in Excavating Classical Culture, ed. by M. Stamatopoulou - M. Yeroulanou, Oxford 2002, 179-86 ha riaffermato l'iniziativa temistoclea. Sul dibattito v. ora M.C. MONACO, La cinta muraria post-persiana e il Pelargikón basso, in GRECO, Topografia di Atene, 75, 78.

296 Così JÜNGER, Gespann und Herrschaft, 236-37, v. n. 290.

297 WeBER, гтKA 'EФ EPMHI, 85, 98-99, 108; JudEICH, Topographie, München $1931^{2}, 237$ n. 1.

298 K. JEPPESEN, The Theory of the Alternative Erechtheion, Aarhus 1987 (Acta Jutlandica 53.1, Humanities Series 60), 38-39. 
biando totalmente prospettiva, ha proposto per l'identificazione del "megaron volto ad Occidente" quello che gli archeologi hanno denominato "Building IV", all'estremità orientale dell'Acropoli, cioè dalla parte opposta rispetto alla quadriga, con i ceppi appesi al recinto del santuario di Zeus Polieus. ${ }^{300}$ Sono invece comunque riconducibili all'area del Tempio arcaico di Atena le proposte di J.A. Bundgaard (recinto del Pandroseion ${ }^{301}$ e di S. Bancroft e J.A.S. Evans (il tempio stesso), ${ }^{302}$ peraltro poco compatibili con il termine $\tau$ õ $\chi 0 \varsigma^{303}$

\subsubsection{3) ANCORA DEL VALORE TEMPORALE DI EEIONTI E DELLA TRADUZIONE DEL PASSO ERODOTEO; LE STRADE DELL'ACROPOLI: WEBER, JUDEICH, DÖRPFELD}

Una sorta di dogma è invece, oltre allo spostamento della quadriga, la sua temporanea collocazione esterna, dinanzi ai Propilei: l'idea, lo si è visto, traeva in realtà origine esclusivamente dalle errate premesse grammaticali di Curtius. ${ }^{304}$ Ancora una volta, quindi, Weber è costretto a difendere il valore di presente ovvero di aoristo del participio presente di $\varepsilon \tilde{i} \mu \mathrm{l}$, come già in precedenza Wachsmuth e Stein: ${ }^{305}$ l'esempio erodoteo prescelto (IV 34, 2) dovrebbe essere particolarmente chiaro per

299 Per il contesto v. par. 3.2.11, 3.2.18, 4.

300 N. Robertson, Athena's Shrines and Festivals, in Worshipping Athena: Panathenaia and Parthenon, ed. by J. NeILs, Madison WI 1996, 27-77: 42.

301 J.A. BundgaARD, Parthenon and the Mycenaean City on the Heights, København 1976, 118.

302 S. BANCROFt, Problems Concerning the Archaic Acropolis at Athens, Diss. Princeton University, Ann Arbor MI UMI 1979, 5-6 e J.A.S EvANS, The Quadriga at the Entrance to the Acropolis, «RSC», 27 (1979), 13-15: 14, che vorrebbe mettere in dubbio le prove archeologiche dell'esistenza del muro di sostegno.

303 Già BergK, Poetae Lyrici Graeci, III, 478 aveva avvertito il problema, e si spingeva a proporre di leggere $\tau \varepsilon \imath \chi i \omega v$ così da poter liberamente pensare alle pareti di un tempio o alle macerie che lo cingevano.

304 CurTIUs, Zur Topographie, 53-54; v. par. 3.1.10.

305 WaChsmuth, Der Standort des ehernen Viergespann, 20-23; STEIN, Jahresbericht über Herodot für 1879, 93; v. par. 3.1.14. Meno efficace SCHMOLLING, Herodots Berichte, 691, quando osserva un po' troppo rigidamente che se Erodoto avesse voluto dire "unmittelbar vor dem Eingang", l'avrebbe espresso come a II 169, 4, ove

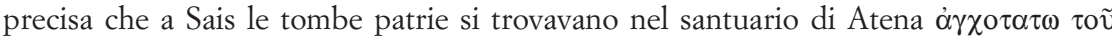

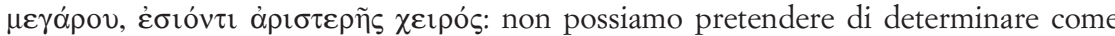
Erodoto avrebbe dovuto scrivere. 
la sua ridondanza (la tomba degl Iperborei si trovava a sinistra entrando nell'Artemisio, con la posizione interna evidenziata da $\check{\varepsilon} \sigma \omega, \dot{\varepsilon} \varsigma$,

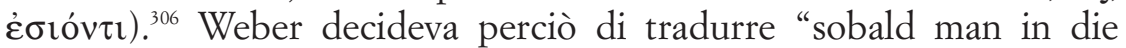
Propyläen eingetreten ist", intendendo la combinazione $\pi \rho \tilde{\omega} \tau o v+$ subordinata implicita come equivalente ad un غ̇ $\pi \varepsilon \dot{i} \pi \rho \tilde{\omega} \tau \alpha+$ subordinata esplicita mediante l'attribuzione a $\pi \rho \tilde{\omega} \tau o v$ di un valore temporale, come già Stein $:^{307}$ in questo modo Erodoto porrebbe se stesso, o un eventuale visitatore, già all'interno dei Propilei; da lì guarderebbe a sinistra, ma anziché dire "la quadriga si vede a sinistra", semplifica l'espressione, scrivendo che "sta a sinistra", ${ }^{308}$ probabilmente vicino, dinanzi alla Promachos, sino a essere, l'originale esattamente come la copia, molto vicina alla strada che passava tra il Tempio arcaico di Atena e quello nuovo, il Partenone in costruzione. ${ }^{309} \mathrm{Ha}$ tuttavia giustamente fatto notare Judeich che, se si pensa alla base nei pressi della Promachos e si considera che la Promachos si trovava sulla destra e non sulla sinistra allo sbocco degli Antichi Propilei, la quadriga si sarebbe dovuta trovare ancora più a destra, e non a sinistra come dice Erodoto, oltrepassando gli Antichi Propilei e percorrendo l'antica via che portava al Tempio arcaico di Atena. ${ }^{310}$ Qui però Weber e Dörpfeld precisano che la Via Processionale era ormai un'altra, quella che passava tra l'antico e il nuovo tempio di Atena ${ }^{311}$ ed effettivamente percorrendola quadriga e Promachos si trovavano sulla sinistra. Quindi, a meno di non rinunciare all'identificazione della base della quadriga con quella immediatamente a Sud-Est della Promachos, ciò che permetterebbe di collocare liberamente il monumento più a Nord-Ovest, ${ }^{312}$ bisognerebbe in pratica tradurre l'indicazione erodotea "una volta entrati nell'Acropoli", più che "entrati nei Propilei": si potrebbe comunque

306 WeBER, Zum Viergespann, 334.

307 STEIN, Jahresbericht über Herodot für 1879, 93-94; v. par. 3.1.15.

308 WEBER, IYKA 'ЕФ EPMHI, 95-96; 100-02: Weber era anche tentato di operare un'ulteriore semplificazione eliminando un articolo e riferendo quindi la determinazione

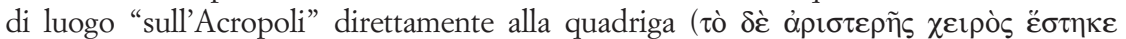

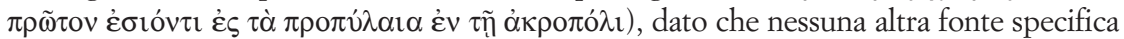

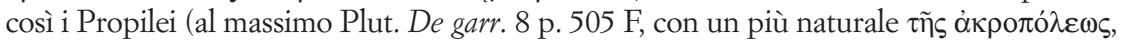
riferito però al termine $\pi \cup ́ \lambda \alpha$, che poteva forse essere maggiormente frainteso).

309 WEBER, гҮKA 'ЕФ EPMHI, 103.

310 JudEICH, Topographie, München 1931², 238.

311 WeBER, Zum Viergespann, 334-36.

312 WEBER, IYKA 'EФ EPMHI, 103. 
spiegare col fatto che Erodoto, dopo essere entrato nei Propilei, si è già messo sulla Via Processionale nuova e si è così imbattuto nella quadriga alla sua sinistra: ${ }^{313}$ già Furtwängler riteneva di essere di fronte ad un'espressione non esattissima da parte di Erodoto, che probabilmente pensava già alla meta, all'entrare nell'Acropoli. ${ }^{314}$

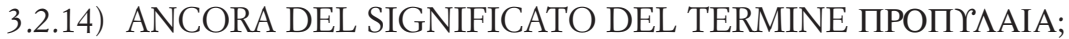 INGRESSI MINORI SULL'ACROPOLI? WEBER, JUDEICH, DÖRPFELD, HOLLAND, RAUBITSCHEK}

L'uso linguistico sconsiglierebbe di interpretare $\tau \grave{\alpha} \pi \rho \circ \pi \dot{\lambda} \lambda \alpha \iota \alpha$ come spazio compreso tra l'ingresso nell'Acropoli ed il muro di sostegno del Tempio arcaico di Atena,${ }^{315}$ non lontano dal quale e quasi in corrispondenza del muro di sostegno del Partenone si sarebbe tra l'altro aperta almeno un'altra porta, nell'ipotesi di Dörpfeld riferita con approvazione da Weber. ${ }^{316}$ Quest'ultimo accenno ad altre porte sarà di lì a poco ripreso e sviluppato, incontrando pure un certo successo.

L.B. Holland ha infatti sostenuto che Erodoto farebbe riferimento ad un'altra entrata presente sull'Acropoli, "an ancient gate now wholly gone", in linea con il muro retrostante la Promachos; in questo modo la quadriga avrebbe potuto ininterrottamente trovarsi alla mano sinistra per chi entrava. ${ }^{317}$ Tuttavia, se è vero che l'ipotesi non si basava su presupposti del tutto indimostrabili (come invece vorrebbe Judeich ${ }^{318}$ e riusciva a riprendere Dörpfeld-Weber senza per questo proporre per $\pi \rho 0 \pi v \dot{\lambda} \alpha \alpha \alpha$ un significato non attestato, ${ }^{319}$ tentando anche di spiegare la curiosa determinazione dei Propilei $\tau \grave{\alpha} \dot{\varepsilon} v \tau \tilde{\eta} \dot{\alpha} \kappa \rho о \pi o ́ \lambda \iota$ come riferimento a delle porte interne, cionondimeno fuori luogo appare l'ottimismo successivamente espresso in proposito da Raubitschek:

313 WEBER, Zum Viergespann, 336.

314 FuRTWÄNGLER, Meisterwerke, 15.

315 Una sorta di riedizione al contrario della proposta di Stein (Herodotos erkl. STEIN, I.1, Berlin $1883^{5}$, XXII n. 1), che pensava allo spazio esterno.

316 DÖRPFELD in WeBER, Zum Viergespann, 334-36.

317 L.B. Holland, The Chariot at the Gates of the Acropolis, in General Meeting of the Archaeological Institut of America, December 27-29, 1923, «AJA», 28 (1924), 6780: 77.

318 JuDEICH, Topographie, München $1931^{2}, 237$.

319 DÖRPFELD in WeBER, Zum Viergespann, 334-36. 
"Herodotos may very well have referred to this building" ${ }^{320}$ Le fondazioni di un simile Propileo evidenziate da Stevens, ${ }^{321}$ infatti, riguardano un ingresso sulla stessa linea del muro di rinforzo del Tempio arcaico di Atena, ma in direzione perpendicolare, ciò che già implicherebbe un cambiamento repentino e totale nell'orientamento nel momento stesso in cui si dovrebbe dare un punto di riferimento; anche il participio presente di $\varepsilon \tilde{i} \mu \mathrm{r}$ andrebbe poi conseguentemente e forzatamente - interpretato come un vero e proprio futuro. Ma più di quelle che possono più o meno a ragione sembrare sottigliezze, appare decisiva la considerazione che un'entrata ad uno dei recinti sacri dell'Acropoli ${ }^{322}$ difficilmente potesse costituire un punto di riferimento valido, pur introducendo ufficialmente al Partenone: il signi-

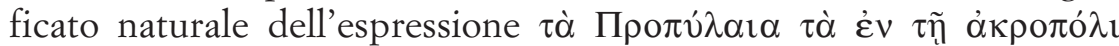
dovrebbe essere l'entrata principale dell'Acropoli, non un ingresso posto di lato come questo, ${ }^{323}$ la cui costruzione peraltro risalirebbe, con quella del muro corrispondente, all'età periclea. ${ }^{324}$ Un altro ingresso, difficile da ricostruire ma comunque molto modesto, poteva introdurre al temenos del Tempio arcaico di Atena ed in seguito alla zona dell'Eretteo: in nessun modo può essere messo in relazione con la localizzazione erodotea della quadriga, per dimensioni e posizione (molto più a Est lungo la Via Processionale, "on the axis of the Porch of the Maiden" $)^{325}$ come sembra fare Raubitschek in alcuni dei suoi confronti; egli si spinge anzi a presupporre, senza alcuna prova archeologica, un ulteriore Propylon a regolare l'accesso alla Via

320 RaubiTSCheK, Dedications, 204: su Arist. Ath. 15, 4 e Polyaen. I 21, 2, citati da Raubitschek a sostegno della sua identificazione, si discute ancora (v. ad es. N.

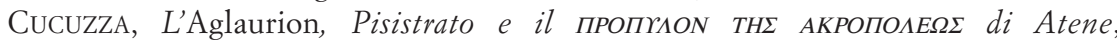
«AION(archeol)», n.s. 3 (1996), 91-97, con bibliografia delle proposte precedenti), ma il riferimento è in ogni caso a porte che si aprivano nella cinta muraria dell'Acropoli, non al suo interno.

321 Stevens, The Periclean Entrance Court, 476-80.

322 Sui quali v. M. KorREs, The History of the Acropolis Monuments, in Acropolis Restoration. The CCAM Interventions, ed. by R. ECONOMAKIS, London 1994, 34-51: 39.

323 R. MeIGGS - D. LEWIS, A Selection of Greek Historical Inscriptions to the End of the Fifth Century B.C., Oxford 1969, $29 \mathrm{n}^{\circ} 15$.

324 Hurwit, The Athenian Acropolis, 216.

325 G. Ph. Stevens, The Setting of the Periclean Parthenon, Athens 1940 (Hesperia Suppl. 3), 42-43. 
Processionale: ${ }^{326}$ ma un Propileo del genere, oltre ad essere indimostrabile, visto che non ha lasciato alcuna traccia, sarebbe soprattutto privo di senso, perché non costituirebbe l'entrata ad alcun temenos, come invece tutti gli altri a cominciare dai Propilei veri e propri. In ogni caso, non è congetturando strutture diverse da questi ultimi (Antichi o Nuovi) che il problema della collocazione topografica della quadriga può essere risolto.

Resta invece a Weber il merito di aver ricapitolato e rafforzato le ragioni della continuità contro le ipotesi di successivi spostamenti, anche all'esterno, della dedica.

\subsubsection{5) UNA SOLUZIONE IMPOSSIBILE (LO SPOSTAMENTO DEI CEPPI): HILLER VON GAERTRINGEN}

D'altro canto vi è stato chi ha immaginato spostamenti ben più paradossali; scriveva infatti testualmente F. Hiller von Gaertringen: "priscum epigramma prope vincula fuerat et ea potissimum (v. 1) respicit. Post Persarum invasionem vincula in interiorem arcis partem translata sunt". ${ }^{327}$ Egli non solo accoglieva l'ipotesi non particolarmente necessaria, ${ }^{328}$ eppure piuttosto diffusa, di uno spostamento segnalato dalla trasposizione dei versi dell'epigramma, ${ }^{329}$ ma attribuiva questo presunto spostamento a ciò che nemmeno nelle congetture degli studiosi si era mai mosso: i ceppi, che Erodoto (V 77, 3) dichiarava esplicitamente di aver visto nel luogo in cui erano stati fin dall'inizio dedicati. Del resto Hiller, dopo aver ingiustamente accusato di inaccuratezza Pausania sulla scorta di Robert, ${ }^{330}$ concludeva segnalando in modo piuttosto confuso come "pristinum locum" (per la quadriga) il taglio

326 RaubitscheK, Dedications, 204. L'ipotizzato Propylon si sarebbe trovato secondo Raubitschek tra i muri b e d in STEVENS, The Periclean Entrance Court, 445 fig. 1, per analogia con i due ingressi $\mathrm{n}^{\mathrm{i}} .7$ e 25 in STEVENS, The Setting, 2 fig. 1 (con il n. 25 nettamente meno sicuro).

327 Inscriptiones atticae Euclidis anno anteriores, ed. F. HILLER DE GAERTRINGEN, Berolini 1924, 190 n $^{\circ} 394$.

328 V. par. 3.2.17. L'ipotesi, come sappiamo, era stata formulata da KIRCHHOFF (LolLING), Bemerkungen zu dem Bruchstück, 114; v. par. 3.2.1.

329 "Eine gewisse topographische Bedeutung" vi assegna JudEICH, Topographie, München $1931^{2}, 237$.

330 ROBERT, Pausanias, 94-95. 
nella roccia che Weller e Judeich avevano assegnato al momento della renovatio. ${ }^{331}$

\subsubsection{6) LA TEORIA DELLO SPOSTAMENTO DELLA QUADRIGA E LE INDAGINI ARCHEOLOGICHE: STEVENS}

La soluzione ancor oggi comunemente accettata consiste invece, come abbiamo già avuto modo di vedere, nella teoria dello spostamento della quadriga (l'originale interno all'Acropoli è sostituito dopo la distruzione persiana da una copia posta all'esterno dell'Acropoli, poi trasferita all'interno), delineata e via via perfezionata da Kirchhoff, Hauvette, Weller, Judeich: anch'essa deve tuttavia convivere con le sue incongruenze linguistiche e soprattutto religiose, ma ha ricevuto un grandissimo impulso - sino ad essere da molti considerata l'unica soluzione - a partire dalle indagini archeologiche di Stevens: egli infatti dichiarò di aver individuato i siti successivamente occupati dalla quadriga.

Basandosi dunque sulla teoria dello spostamento egli proponeva per la prima fase, interna all'Acropoli, un taglio nella roccia nei pressi della Promachos, ma con orientamento diverso da essa (non vengono però specificate le misure del taglio). ${ }^{332}$ Per la seconda fase, esterna all'Acropoli, Stevens riprendeva in un primo momento in considerazione il sito del monumento ad Agrippa, attribuendo quindi il trasferimento alla costruzione di quest'ultimo, oppure, ancor più infelicemente, ad una non meglio specificata nuova vittoria su Beoti e Calcidesi nel 330 a.C., ${ }^{33}$ che non trova alcun riscontro. Dal punto di vista storico, infatti, l'unico episodio vagamente simile cui si potrebbe eventualmente pensare si verifica non nel 330, ma nel 304 a.C., quando Demetrio Poliorcete, chiamato in aiuto dagli Ateniesi, libera Calcide proprio da una guarnigione beotica, imposta da Cassandro. ${ }^{334} \mathrm{P}$. Cloché aggiunge

331 Weller, The Pre-Periclean Propylon, tav. I, lettera K; JudeICH, Topographie, München 1905, 198 fig. 22.

332 Stevens, The Periclean Entrance Court, 492 fig. 42 taglio non numerato immediatamente a Sud del n 2 ("rectangular cutting immediately south of 2, figure 42", 506).

333 Stevens, The Periclean Entrance Court, 505.

334 Diod. XX 100, Plut. Demetr. 23. 
anche che Demetrio costrinse i Beoti a rinunciare a Eretria e a lasciare Oropo agli Ateniesi, senza però citare la fonte di questa affermazione. ${ }^{335}$ La questione di Oropo era stata però trattata da G. De Sanctis, ${ }^{336}$ il quale a sua volta si basava sull'allora recentissima pubblicazione di un decreto onorario ateniese, 337 "che pare supporre il dominio ateniese in Oropo, datato dall'arconte Leostrato; arconte di data sicura (303/2), il penultimo di cui ricorra il nome in Diodoro", ${ }^{338}$ mentre la sottrazione ai Beoti anche di Eretria oltre a Calcide è semplicemente un'ipotesi, che fa anche P. Roesch; ${ }^{39}$ tutti sono d'accordo, poi, nel ricordare che Demetrio prese anche File, Panatto e Salamina e le restituì agli Ateniesi. ${ }^{340}$ Sono questi i risultati che potrebbero essere letti come un successo per Atene sui Beoti e sui Calcidesi in quel periodo, ma in verità più per opera di Demetrio Poliorcete che degli Ateniesi. Del resto l'ipotesi non è stata più precisata da Stevens che anzi l'ha lasciata cadere. Egli ha invece insistito nella ricerca di un sito esterno per il rifacimento della quadriga, dinanzi ai Propilei, giungendo alla conclusione che una serie di blocchi di poros, di orientamento diverso dai Propilei di Mnesicle e da essi parzialmente ricoperti, avrebbe potuto costituire la base per la quadriga rinnovata, rimanendo sempre a sinistra per chi saliva le rampe che portavano ai Propilei. ${ }^{341}$ A questo proposito, però, W.K. Pritchett, consultatosi con J. Binder e T. Tanoulas, ha precisato, pur condividendo la teoria dello spostamento, che la situazione del sito sottostante i Propilei di Mnesicle proposta per la seconda fase di vita della dedica non è esattamente quella descritta da Stevens, visto che $\mathrm{i}$ blocchi di poros di cui parlava non si trovano sul sito indicato, ${ }^{342} \mathrm{ma}$

335 P. Cloché, Thèbes de Béotie: des origines à la conquête romaine, NamurLouvain-Paris 1952, 206.

336 G. De SANCTIS, Oropo, «RFIC», n.s. 4 (1926), 222-23.


102: 86-89 (= SEG III 26-27 $\mathrm{n}^{\circ} 117$; ISE, I, 8). Altre considerazioni a sostegno dell'ipotesi in L. RoBERT, Hellenica, XI-XII, Paris 1960, 201-02; tutta la questione esposta con chiarezza in L. MORETTI, Iscrizioni Storiche Ellenistiche, I, Firenze 1967, 15-17.

338 De SANCTIS, Oropo, 222.

339 P. Roesch, Études béotiennes, Paris 1982, 429, cfr. M. Holleaux, Études d'épigraphie et d'histoire grecques, I, Paris 1938, 58 n. 3.

340 Plut. Demetr. 23; Paus. I 35, 2.

341 STEvens, Architectural Studies, 78 fig. 4 n ${ }^{\circ}$ 20; 81-82.

342 STEVEnS, Architectural Studies, 78 fig. 4 n 20. 
sono stati riutilizzati per le fondazioni dei Propilei; vi sarebbe comunque un taglio nella roccia adatto per la quadriga. ${ }^{343}$ Infine, concludeva Stevens, poiché le fondazioni dei Propilei di Mnesicle coprono questa zona, alla loro costruzione la quadriga restaurata sarebbe stata spostata fra la Promachos e i Propilei, dove l'avrebbe vista Pausania: candidato per la terza fase, nuovamente interna all'Acropoli, sarebbe un taglio nella roccia che si trova circa $8 \mathrm{~m}$ a Nord-Est del portico orientale dei Propilei; il taglio ha questa volta lo stesso orientamento della Promachos e, a detta di Stevens, si sarebbe potuto adattare in larghezza e profondità alle probabili dimensioni della quadriga (ricostruibili, è vero, con buona approssimazione: ma è del taglio che anche qui non vengono specificate le misure); ${ }^{344}$ il trasferimento sarebbe comprovato dal fatto che questo taglio si trova nelle vicinanze di quello della quadriga originale ma è di diverso orientamento e di epoca più recente. ${ }^{345}$

Ora, anche senza contare i dubbi espressi sulla compatibilità della forma di questi tagli, in particolare del taglio esterno, i cui bordi ricurvi farebbero addirittura escludere che potesse accogliere la base di una statua, ${ }^{346}$ è evidente che i siti individuati non costituiscono delle prove per la teoria dello spostamento: semplicemente non la contraddicono. E' noto, del resto, l'affollarsi di dediche all'interno dell'Acropoli: ${ }^{347}$ siamo quindi liberi di assegnare i tagli via via identificati ai monumenti più diversi, non necessariamente a quelli più studiati. Lo stesso Stevens, ad esempio, congettura, sul luogo che avrebbe secondo lui successivamente accolto una piattaforma terrazzata che portava alla statua di Atena Promachos, un piccolo edificio sacro ("shrine"), delineato da quattro tagli nella roccia, ${ }^{348}$ del quale in realtà nulla sappiamo: forse che i medesimi tagli non avrebbero potuto accogliere monumenti diversi da quello ipotizzato, magari proprio la nostra quadriga in una fase anteriore, visto che le dimensioni sembrano coincidere con quelle del sito

343 W.K. PritchetT, The Liar School of Herodotos, Amsterdam 1993, 157.

344 Stevens, The Periclean Entrance Court, 492 fig. 42 n 2; 506; STEVEns, Architectural Studies, 81-82.

345 Stevens, The Periclean Entrance Court, 506.

346 Mylonas Shear, The Western Approach, 106 con n. 116.

347 Miller, A History of the Akropolis, 503; KeEsLing, The Votive Statues, 12; J. Pedley, Sanctuaries and the Sacred in the Ancient Greek World, Cambridge 2005, $189-90$

348 Stevens, The Periclean Entrance Court, 492 fig. 42 n ${ }^{\mathrm{i}}$ 10, 11, 12, 13; 509-10. 
proposto da Stevens per la successiva terza fase di vita dell'anathema? ? $^{349}$ Allo stato delle nostre conoscenze sarebbe più prudente evitare di costruire ipotesi sulla base di altre ipotesi. E' chiaro che Stevens ha ricondotto alla quadriga per la vittoria sui Beoti e i Calcidesi tre dei numerosi tagli dell'Acropoli perché seguiva la teoria dello spostamento, che prevedeva, una dopo l'altra, due o tre collocazioni, con quella esterna unicamente desunta dal supposto significato futuro del participio di عĩur; non avesse Stevens seguito quella teoria, il taglio identificato sarebbe stato uno solo (sul quale non si può comunque raggiungere la certezza), con la facoltà di lasciare indeterminata la funzione degli altri due - come spesso accade, in mancanza di dati sicuri - oppure di attribuirli ad altri monumenti, senza che ciò costituisse un problema. E' quindi rischioso concludere che le indagini archeologiche di Stevens hanno confermato il quadro storico-letterario della teoria dello spostamento, dato che è quest'ultima ad aver ispirato le indagini archeologiche; il rischio, in particolare, è quello di un circolo vizioso: dalla ricostruzione storico-letteraria - partita già con premesse discutibili quali l'idea stessa dello spostamento e la collocazione esterna sulla base del participio di $\varepsilon \tilde{i} \mu \mathrm{r}$ - alla indagine archeologica condizionata dalla ricostruzione storico-letteraria e di nuovo a quest'ultima, con l'impressione di aver trovato conferma nell'indagine archeologica. Né valore di prova possono avere considerazioni di tipo estetico, secondo le quali la quadriga sarebbe stata di grande effetto scenografico se avesse occupato il taglio fuori del'Acropoli, ${ }^{350}$ in una collocazione tutta da dimostrare (e poco probabile, si è detto, dal punto di vista religioso).

\subsubsection{7) ANCORA DEL MUTATO ORDINE DEI VERSI NELLA DEDICA: RAUBITSCHEK, SCHOLLMEYER, NENCI, ERBSE, FRIEDLÄNDER-HOFFLEIT}

Infine il valore topografico dell'inversione dell'ordine degli esametri nella coppia di distici dell'epigramma dedicatorio, sostenuto a partire da Kirchhoff, ${ }^{351}$ è stato definitivamente revocato in dubbio, ciò che costituisce il contributo più significativo (e duraturo) nel dibattito

349 Stevens, The Periclean Entrance Court, 492 fig. $42 \mathrm{n}^{\circ} 2$.

350 Stevens, Architectural Studies, 82; Thallon Hill, The Ancient City, 181.

351 KirCHHOFF (Lolling), Bemerkungen zu dem Bruchstück, 114; v. par. 3.2.1. 
che è seguito agli articoli di Stevens. Di Raubitschek è particolarmente apprezzabile, infatti, l'osservazione secondo la quale con la trasposizione l'enfasi passava semplicemente dai ceppi alla vittoria militare: il monumento originale era stato effettivamente eretto, e lo si sottolineava, con la decima del riscatto dei prigionieri in ceppi, mentre il denaro per il rifacimento doveva provenire dal bottino della recente vittoria a Enofita (457 a.C.), o da un fondo più generale, come nel caso dell'Atena Promachos, presso la quale, tra l'altro, veniva a trovarsi la quadriga rinnovata (certo se non se ne ipotizza lo spostamento). ${ }^{352}$ In altre parole, il riferimento ai ceppi non era ormai rilevante, mentre lo era il riferimento alla vittoria ${ }^{353}$ (sui Beoti). La spiegazione è stata poi precisata da Schollmeyer, che ha ricordato come in età arcaica fosse più importante il bottino, in età classica la battaglia stessa. ${ }^{354} \mathrm{Di}$ qui l'ordine differente, senza alcuna necessità di immaginare una temporanea collocazione lontano dai ceppi.

Già Kirchhoff aveva peraltro lasciato aperta la possibilità di una variazione puramente casuale; ${ }^{355}$ Nenci ha quindi pensato che "la differenza fra le due iscrizioni, ipotizzando che la prima fosse andata distrutta durante l'occupazione persiana dell'Acropoli, potrebbe essere dovuta al fatto che se ne ricordavano i versi a memoria, ma non necessariamente nell'ordine originario". ${ }^{356}$

Ad una spiegazione ancora diversa e più complessa è pervenuto H. Erbse: lo studioso, convinto dell'autenticità simonidea dell'epigramma, ${ }^{357}$ dubita che la trascrizione di Erodoto dal monumento abbia

352 RaubitscheK, Dedications, 203.

353 MeIgGs - LeWIS, Greek Historical Inscriptions, 29 n 15; eccessiva la replica di SCHOllmeyer, Antike Gespanndenkmäler, 55 n. 296, secondo il quale a questa stregua sarebbe stata necessaria la cancellazione del riferimento ai ceppi: l'epigramma e lo spirito stesso del rifacimento ne sarebbero stati irrimediabilmente compromessi.

354 Schollmeyer, Antike Gespanndenkmäler, 60 n. 322, cfr. CHr. MeIER, Die Rolle des Krieges im klassischen Athen, «HZ», 1990 (251), 555-605.

355 KirCHHOFf (Lolling), Bemerkungen zu dem Bruchstück, 114; v. par. 3.2.1.

356 NENCI, Erodoto, V, 273.

357 ERBSE, Zu den Epigrammen, 224; egli si chiedeva tra l'altro perché gli Ateniesi, essendo ancora in vita Simonide, avrebbero dovuto rivolgersi a qualche poeta sconosciuto per celebrare un evento così significativo come la prima vittoria militare dell'Atene democratica. Per un riassunto della questione dell'autenticità simonidea v. Petrovic, Kommentar, 218-22 e BerTi, La dedica degli Ateniesi, 14-15. 
potuto sostituire tutta la tradizione antica su questa poesia, obliterandone completamente l'originale; per Erbse Simonide avrebbe invece composto fin dall'inizio nell'ordine della copia, ma l'originale sarebbe stato modificato dagli Ateniesi per mettere in evidenza il disprezzo per il nemico; per il ripristino sarebbe stato utilizzato l'ordine originario, seguendo un testo di Simonide, cosa che avrebbe eventualmente potuto

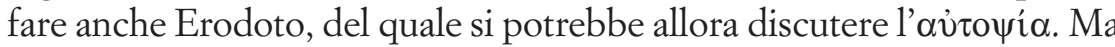
l'ipotesi si basa anche sulla qualità poetica della copia, giudicata migliore, ${ }^{358}$ e la valutazione in merito rimane altamente soggettiva, se si pensa all'ironia sui risultati della trasposizione espressa a suo tempo da $\mathrm{T}$. Preger: "certe hac commutatione constructionem verborum dilucidiorem factam esse nemo, opinor, dicet". 359 Ed anche ammettendo una maggior scorrevolezza della copia (così spiegherebbero l'inversione Friedländer e Hoffleit), ${ }^{360}$ ciò non vuol dire che si tratti in realtà dell'originale: esso poteva benissimo essere caratterizzato da una disposizione delle parole ricercata, o addirittura forzata. ${ }^{361}$

In ogni caso si può concludere che appare azzardato costruire la teoria dello spostamento a partire dall'inversione dei due esametri: le spiegazioni sono molteplici, ed anche a voler presupporre una modifica voluta, questa rimane spiegabile più con ragioni di propaganda (il maggior rilievo dato ai popoli sconfitti) che con un cambiamento del contesto architettonico. ${ }^{362}$ E' chiaro, quindi, che entrambe le communes opiniones topografiche, lo spostamento della quadriga e la sua tempo-

358 ERBSE, Zu den Epigrammen, 225.

359 Inscriptiones Graecae Metricae ex scriptoribus praeter Anthologiam collectae,

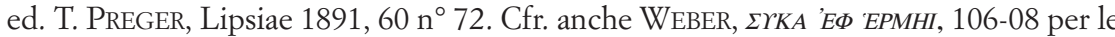
osservazioni stilistiche, eccessive ma interessanti perché di segno totalmente opposto a quelle di Erbse circa la qualità artistica dell'epigramma di $\mathrm{V}$ secolo.

360 P. FRIEDLÄNDER - H.B. HOFFLEIT, Epigrammata. Greek Inscriptions in Verse from the Beginnings to the Persian Wars, Berkeley-Los Angeles 1948, $136 \mathrm{n}^{\circ} 145$

361 Basti ad esempio pensare alla celebre parodia aristofanesca della lingua della poesia corale in Aves, 904-57, cfr. A. MeILLET, Lineamenti di storia della lingua greca, Torino 1976 (trad. it. di A. MeILlet, Aperçu d'une histoire de la langue grecque, Paris $\left.1935^{4}\right), 262-64$.

362 Nella linea di un'interpretazione "topografica" dell'inversione degli esametri si è infatti riusciti a dare anche un senso diametralmente opposto a quello consueto: Stevens in un primo momento aveva l'impressione che la mancata enfasi sui ceppi significasse la loro vicinanza, mentre l'enfasi fosse necessaria in quanto meno visibili al lettore dell'iscrizione (cfr. STEvens, The Periclean Entrance Court, 505)! 
ranea collocazione esterna all'Acropoli, poggiano su basi assai poco solide.

\subsubsection{8) L'AREA DEL TEMPIO ARCAICO DI ATENA E LE DEDICHE PER LE VITTORIE DELLA CITTA': PREIßHOFEN}

Un contributo, a torto trascurato, alla localizzazione della quadriga ha dato F. Preißhofen, indagando la percezione del Tempio arcaico di Atena come centro di culto, ove dedicare i trionfi della città alla divinità protettrice. ${ }^{363}$ Due testimonianze in particolare attirano la sua attenzione: uno scolio alla Lysistrata di Aristofane e un passo di Plutarco. Il primo (Schol. in Aristoph. Lys. 273), su cui torneremo più avanti, ${ }^{364}$ ricorda la condanna a morte di oppositori interni ad Atene, che avevano sostenuto il tentativo di Cleomene spartano di occupare la città, i nomi dei quali furono iscritti su una stele bronzea, che sarebbe stata eretta proprio nei pressi del Tempio arcaico di Atena. Il secondo (Plut. Cim. 5, 3) ci informa che tutt'intorno al Tempio erano appesi degli scudi, evidentemente trofei strappati al nemico. Non può sfuggire l'analogia con i ceppi, ugual-

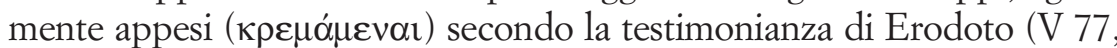
3). L'area del Tempio arcaico di Atena si identifica quindi come luogo ove presentare i trofei della città offerti alla divinità poliade. Di conseguenza non poteva esservi luogo più adatto per l'esposizione della quadriga: Preißhofen dava anzi per scontato che la quadriga si trovasse lì, avendo invece come obiettivo principale la dimostrazione dell'unicità di un centro di culto in età arcaica; ${ }^{365}$ su questo però, come abbiamo visto, si discute, ${ }^{366}$ ed è forse il motivo per cui il contributo dello studioso non è in genere preso in considerazione per la localizzazione della quadriga.

\subsubsection{9) ERODOTO E ATENE: VERA AUTOPSIA? ANCORA SU ERODOTO E LA TOPOGRAFIA: PODLECKI, S. WEST, PRITCHETT, MATTINGLY}

Un rinnovato dibattito, che coinvolse la questione della quadriga

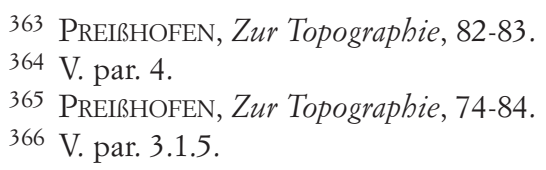


e della sua collocazione, si accese invece intorno al problema del soggiorno ateniese di Erodoto. Particolarmente scettico in materia si mostrava A.J. Podlecki: secondo lo studioso la descrizione del monumento alla vittoria sui Beoti e sui Calcidesi sarebbe anzi la prova che Erodoto non era testimone oculare di ciò che narrava di Atene. ${ }^{367}$ Podlecki, già insospettito dal cambiamento dell'ordine dei versi nella dedica (cambiamento come sappiamo di certo non imputabile a Erodoto) mette infatti in contrapposizione Erodoto e Pausania, scrivendo che quest'ultimo avrebbe visto la quadriga vicino alla Promachos, chiedendosi poi "Why does Herodotus place it "outside the propylaia?". Ora, in nessun luogo Erodoto parla di una collocazione esterna all'Acropoli: si tratta di una (errata, come abbiamo visto) deduzione di alcuni studiosi; eppure è per questo motivo che Podlecki pensa ad un informatore che abbia dato ad Erodoto un resoconto confuso, con una trascrizione però accurata dell'epigramma rifatto, e non ammette un soggiorno ateniese dello storico, se non brevissimo. ${ }^{368}$ In questo modo Erodoto non avrebbe più valore topografico, come già proposto a suo tempo, ma con maggiore consapevolezza e meno estremismo, da Bachof ${ }^{369}$ e dall'ultimo Wachsmuth. ${ }^{370} \mathrm{Se}$ appare condivisibile la cautela di Podlecki nel concludere, di fronte a espressioni erodotee come "fino ai miei tempi" o "tuttora", che Erodoto fosse in tutti casi testimone oculare, ${ }^{371}$ non è tuttavia accettabile che la communis opinio, secondo cui Erodoto utilizzerebbe il participio presente di un composto di zĩur allo scopo di descrivere un monumento come situato dinanzi all'ingresso dell'Acropoli, invece di essere esclusa finisca per causare l'esclusione di Erodoto.

Proprio questo è infatti il risultato cui giunge $\mathrm{S}$. West, che riprende le considerazioni di Podlecki senza tuttavia arrivare a sostenere che Erodoto non sia mai stato ad Atene: secondo la West dalle parole di Erodoto si dovrebbe arguire una posizione della quadriga interna ai

367 A.J. PODLECKI, Herodotus in Athens?, in Greece and the Eastern Mediterranean in Ancient History and Prehistory. Studies Presented to Fritz Schachermeyr on the Occasion of His Eightieth Birthday, ed. by K.H. KINZL, Berlin-New York 1977, 246-65.

368 PODLECKI, Herodotus, 259-63.

369 BACHOF, Zu Herodotos, 177-82; v. par. 3.1.17.

370 WACHSMUTH, Einleitung, 512 n. 1; ID., Bemerkungen zu griechischen Historikern, 215; ID., Athenai, 203-04; v. par. 3.2.7.

371 PODLECKI, Herodotus, 262-63. 
Propilei o nelle immediate vicinanze all'esterno, cosa che mal si concilierebbe con Pausania; la testimonianza di Erodoto andrebbe quindi squalificata, non però per mancanza di autopsia, bensì per semplice imprecisione. ${ }^{372}$ Ecco perché non sarebbe necessario ricorrere alla complicata teoria dello spostamento. Erodoto compirebbe infatti - qui come altrove - un errore di localizzazione, né si potrebbe pretendere che prendesse puntuali annotazioni topografiche, avendo maggiore memoria per la poesia piuttosto che per la topografia: infatti, "these lines add no further factual details to his narrative, but they provide a stately, formal conclusion to his account of the extraordinary success which followed Athens' liberation from tyranny, illustrating one of his favourite ideas, the stimulating effect of political change". ${ }^{773}$ Ancora una volta, quindi, l'accettazione di una communis opinio causava un forzato contrasto tra le fonti, sino ad escludere una di esse; eppure, se la conclusione ad effetto da parte di Erodoto è innegabile, non per questo si deve pensare che allo storico importasse esclusivamente l'estetica, altrimenti sarebbe stato molto più vago nelle sue indicazioni, parlando semplicemente di "Acropoli" come fa Diodoro, senza specificare "a sinistra per chi entra nei Propilei": perché Erodoto si sarebbe ostinato a precisare se non ricordava bene la posizione della quadriga? Una buona parte del suo pubblico poteva conoscere Atene e avrebbe capito comunque senza ulteriori precisazioni (e notato errori grossolani): dunque Erodoto era probabilmente sicuro di quanto scriveva.

Alle teorie di Podlecki e della West si oppone Pritchett, che fa notare come non si possa accusare di inaccuratezza Erodoto sulla scorta di Pausania, che vide l'Acropoli più di cinque secoli e mezzo dopo. Nel frattempo Atene era stata saccheggiata da Silla. Inoltre gli Ateniesi, nel corso della loro storia, dalle origini all'età romana, amarono cambiare e spostare strade, servizi, monumenti, templi: per esempio in occasione della costruzione dei nuovi Propilei. La stessa sorte potrebbe essere toccata alla nostra quadriga. ${ }^{374}$ In quest'ultimo caso, però, Pritchett respingerebbe ipotesi costruite sulla communis opinio per mezzo di un'altra communis opinio: l'ipotizzata collocazione esterna della quadriga non sarebbe cioè in conflitto con la testimonianza di

372 S. West, Herodotus' Epigraphical Interests, «CQ», 35 (1985), 278-305: 284.

373 WEST, Herodotus, 284-85.

374 W.K. PRITCheTt, The Liar School, 157-58. 
Pausania grazie all'ipotesi dello spostamento. Pur senza ignorare il dibattito ottocentesco, Pritchett riprendeva poi il significato presente o futuro del participio éoı́v $\tau \iota^{375}$ alla base della possibilità che la quadriga si fosse trovata dinanzi o all'interno degli Antichi Propilei, come suggeritogli da T. Tanoulas: ma si sono già visti gli esempi dell'uso linguistico erodoteo relativi a un significato anche aoristo di un simile participio $^{376}$. Più condivisibile invece il richiamo alla seriorità di Pausania (tra l'altro meno interessato rispetto a Erodoto a dare indicazioni topografiche nel passo in questione).

Quasi nessuno, del resto, esclude più almeno un soggiorno ateniese di Erodoto, ${ }^{377}$ come spiega L. Prandi, infatti, il ricorso a sistemi di riferimento ateniesi e l'assimilazione di problematiche e temi propagandistici ateniesi sono la prova di una mentalità ateniese che, a differenza di singole notizie magari ricavabili anche altrove, può derivare soltanto da una permanenza anche prolungata nella città, non bastando a un estraneo come Erodoto una semplice frequentazione di coloni ateniesi a Turi: il soggiorno ateniese di Erodoto risulta garantito "non tanto per il tipo di informazioni, ma per lo spirito che le informa, per l'articolazione delle idee, per l'effetto di immersione totale nella realtà ateniese" ${ }^{378}$

D'altro canto fin troppo certa appariva a Mattingly la presenza di Erodoto ad Atene dopo il 431, impegnato nel completamento degli ultimi libri: il secondo soggiorno ateniese costituiva infatti il presupposto per

375 PRITCHETT, The Liar School, 157 n. 125 appoggiandosi exempli gratia a H.W. SмYTH, Greek Grammar, rev. by G.M. Messing, Cambridge MA 1956 (= 198011), 422 $\$ 1880$.

376 V. par. 3.1.14 e 3.2.5.

377 Sulla biografia di Erodoto fondamentale F. JACOBY, Herodotos, in RE, Suppl. II (1913), 205-520: 205-80 (in particolare Erodoto ad Atene: 236-39; non è possibile decidere se un secondo soggiorno abbia avuto luogo o meno: 239; 243-47); più sinteticamente Erodoto. Le Storie, I, intr., testo e comm. a c. di D. AsHERI, trad. a c. di V. Antelami, Milano 1988, XII-XIV e K. MeIster, Herodotos, in DNP, V (1998), 469-75: 469-70; non esclude il secondo soggiorno ateniese R. FOWLER, Herodotos and Athens, in Herodotus and His World. Essays from a Conference in Memory of George Forrest, ed. by P. DEROw - R. PARKer, Oxford 2003, 305-18: 305-06; per la storicità dei viaggi di Erodoto (contro i dubbi di D. FEHLING, Die Quellenangaben bei Herodot. Studien zur Erzäblkunst Herodots, Berlin 1971, 168-70 e passim) v. ora p.es. D. MüLlER, De Herodoti itineribus nuper retractatis, «VoxLat», 33 (1997): 310-327.

378 L. PRAnd, Tre questioni erodotee, «Aevum(ant)», 4 (1991), 77-114: 78-81. 
la teoria secondo la quale Erodoto farebbe riferimento ai Propilei di Mnesicle e alla copia della quadriga, che, rimpiazzato l'originale già negli anni '70 ma ancora all'interno dell'Acropoli, sarebbe stata invece definitivamente collocata dinanzi ai Propilei, in occasione, per Mattingly, del fallito tentativo tebano di conquistare Platea nel $431 .^{379} \mathrm{Il}$ presupposto, come si sa, è discusso, ${ }^{380}$ la datazione del ripristino poco probabile, ${ }^{381}$ Pausania, infine, non viene neppure preso in considerazione.

\subsubsection{0) LE DUE TESI PIÙ DIFFUSE NEL DIBATTITO ATTUALE: SPOSTAMENTO O CONTINUITA'?}

Negli ultimi trent'anni la grande maggioranza degli studiosi ha affrontato il problema della collocazione topografica della quadriga semplicemente aderendo all'ipotesi del suo spostamento; solo una minoranza ha rinnovato le obiezioni, che rendono invece più probabile l'ipotesi di un ripristino del monumento nel sito originario e una sua successiva premanenza nel medesimo luogo.

Al primo gruppo si potrebbero ascrivere solo parzialmente gli studiosi nominati nel paragrafo precedente, in quanto autori di proposte più elaborate, che hanno quindi richiesto una presentazione separata. $\mathrm{Ne}$ fa invece parte, per esempio, Evans, che considera lo spostamento della quadriga, di cui Erodoto non sarebbe stato consapevole (i Propilei nominati non sarebbero quelli di Mnesicle), l'unica certezza nel dibattito in corso. ${ }^{382}$ Molto chiara è poi l'esposizione della teoria dello spostamento ad opera di L. Beschi3 ${ }^{383}$ e successivamente di N. Modenesi. ${ }^{384}$ Diversamente M. Korres sembra addirittura tornare a Curtius ${ }^{385}$ o a Körte $^{386}$ quando scrive che già la dedica originaria sarebbe stata posta

379 H.B. MatTingly, The Athena Nike Temple Reconsidered, «AJA», 86 (1982), 381-86: 383-84 (= ID., The Athenian Empire Restored, 466-68)

380 V. n. 376.

381 V. BERTI, La dedica degli Ateniesi, 32-33.

382 Evans, The Quadriga, 14-15.

383 Pausania, I, a c. di BeSCHI - Musti, 367; più sintetico BeSCHI, Atene, 503, già citato in apertura.

384 Modenesi, Pausania, 27-29.

385 CurTius, Zur Topographie, 53-54; v. par. 3.1.10.

386 KÖRTE, recensione a E. PETERSEN, 844-45 n. 1; v. par. 3.2.10. 
"just outside the western gate of the still-preserved Mycenaean walls";37 più prudentemente J.M. Hurwit ammette che non conosciamo l'originaria collocazione della quadriga, ma riprende comunque la teoria dello spostamento, ${ }^{388}$ come del resto fa C.M. Keesling. ${ }^{389}$ Nel solco di questa tradizione, infine, si colloca pure l'articolato studio di Jünger, ${ }^{390}$ che almeno per la localizzazione originaria della nostra quadriga ha coraggiosamente proposto un sito diverso da quelli ormai consolidati nell'opinione corrente: la terrazza del Tempio arcaico di Atena, al di là del muro di sostegno, che viene qui ricostruito come vero e proprio recinto sacro maggiormente sviluppato in altezza, ${ }^{391}$ dal quale pendono i ceppi; la communis opinio viene tuttavia seguita per le fasi successive separazione della quadriga dai ceppi e ritorno nelle loro vicinanze - giustificando anzi in un circolo vizioso l'esposizione all'esterno dell'acropoli con la perdita, a giudizio di Jünger, del carattere sacrale della dedica, perdita di sacralità che sarebbe appunto dimostrata (ecco il circolo vizioso) dall'ostentato trasferimento all'esterno. ${ }^{392}$ Né a questo punto si comprende per quale motivo gli Ateniesi avrebbero dovuto rinnovare l'iscrizione dedicatoria ad Atena, se veramente non si trattava più di una dedica!

Altri studi recenti hanno invece sostenuto nuovamente, dopo la difesa di Preißhofen, ${ }^{393}$ le ragioni della continuità. Schollmeyer sottolinea che una dedica del popolo ateniese ad Atena sarebbe impensabile fuori del recinto sacro della divinità e colloca quindi l'originale della fine del VI secolo, come pure la copia da metà del V all'età romana quando la vide Pausania, sempre fra gli Antichi Propilei e il Tempio

387 KORRES, The History, 41; similmente L. GIULIANI, Die Giganten als Gegenbilder der attischen Bürger im 6. und 5. Jabrbundert v. Chr., in Gegenwelten zu den Kulturen Griechenlands und Roms in der Antike, hrsg. von T. HÖLSCHER, MünchenLeipzig 2000, 263-86: 281.

388 HuRWIT, The Athenian Acropolis, 146.

389 KeEsling, The Votive Statues, 14.

390 JüNGER, Gespann und Herrschaft, 224-49.

391 JÜNGER, Gespann und Herrschaft, 239, 240 fig. 39.

392 JÜNGER, Gespann und Herrschaft, 243: unico scopo sarebbe stata l'universalizzazione della vittoria. Anche il supposto ritorno all'interno dell'Acropoli viene spiegato con ragioni extra-religiose: la quadriga sarebbe stata accostata alla Promachos come su un palcoscenico (JÜNGER, Gespann und Herrschaft, 248). Su considerazioni di carattere estetico come queste (peraltro non nuove) v. par. 4.

393 PReißhofen, Zur Topographie, 82-83; v. par. 3.2.18. 
arcaico di Atena; non ritenendo però strettamente necessario che ceppi e quadriga fossero esposti assieme, distingue la dedica del monumento dai ceppi considerati dei trofei: ${ }^{394}$ una considerazione, quest'ultima, discutibile, visto che l'epigramma dedicatorio, come si sa, contiene un importante riferimento ai ceppi. Per la contiguità fra quadriga e ceppi ed anche per la permanenza nel medesimo luogo, per motivi religiosi, interno all'Acropoli, di fronte alla cella occidentale del Tempio arcaico di Atena, si è espresso E. Kluwe. ${ }^{395} \mathrm{Da}$ ultimo Petrovic ha rivalutato le indicazioni topografiche erodotee, a prezzo però di escludere del tutto il contributo di Pausania alla questione, ${ }^{396}$ tenuto invece ancora in considerazione da M.C. Monaco, pur con estrema cautela ${ }^{397}$.

\section{ALCUNE (RI)CONSIDERAZIONI}

Nel corso di questa sorta di storia ragionata degli studi sulla collocazione topografica della quadriga dedicata dagli Ateniesi per la vittoria sui Beoti e sui Calcidesi è stato possibile seguire la genesi di alcune communes opiniones, in particolare l'ubicazione, più o meno temporanea, dell'anathema all'esterno dell'Acropoli e la conseguente necessità di un suo spostamento successivo. Come si è già avuto modo di osservare, ${ }^{398}$ anche le indagini archeologiche sono state influenzate da premesse non accettabili: la necessità di assegnare alla quadriga un sito esterno all'Acropoli è infatti da ricondurre in ultima analisi all'errata convinzione che il participio presente di عĩur utilizzato da Erodoto per segnalare la posizione del monumento abbia esclusivo significato di futuro, quando sono invece attestati nell'uso, di Erodoto come di altri scrittori, significati di presente e passato. ${ }^{399}$ Allo stesso modo, numerose spiegazioni alter-

394 SCHOLLmeYer, Antike Gespanndenkmäler, 55, 57; gli esempi addotti non sembrano calzanti.

395 KLuWe, Das Siegesmal, 275.

396 Petrovic, Kommentar, 212-13, in questo seguito da N. Livingstone - G. Nisbet, Epigram, Cambridge 2010 (G\&R New Surveys in the Classics 38), 32 n. 32.

397 M.C. Monaco, Le statue dell'Acropoli, in GRECO, Topografia di Atene, 118 21: 119 ("Non scevra da problemi risulta l'originaria ubicazione del donario (...) che, stando al dettato di Pausania, sembrerebbe comunque essersi trovato in prossimità dell'agalma dell'Atena Promachos").

398 V. par. 3.2.8, 3.2.9 e 3.2.16.

399 V. par. 3.1.14 e 3.2.13. 
native fanno escludere che il diverso ordine dei versi nella dedica possa trovare ragione nello spostamento della stessa. ${ }^{400} \mathrm{Si}$ è pure cercato di giustificare la temporanea esposizione della quadriga immediatamente all'esterno dell'Acropoli con ragionamenti di carattere estetico, relativi sia all'effetto scenografico di una collocazione dinanzi ai Propilei sia alla eccessiva vicinanza alla Promachos che si sarebbe determinata nel caso di una collocazione interna all'Acropoli: ma si è già osservato che quest'ultimo sarebbe comunque stato il risultato finale anche per chi ha ipotizzato lo spostamento della quadriga; secondariamente è noto quanto fossero normalmente affollati di dediche tutti i santuari, Acropoli inclusa; $; 01$ infine l'efficacia estetica in generale può non essere particolarmente probante, considerando l'elevato grado di soggettività nel giudizio: Hurwit, addirittura, proprio nel dare conto della teoria dello spostamento giunge a considerazioni estetiche esattamente all'opposto di quelle che lo spostamento dovrebbero giustificare, sostenendo che lo splendore materiale e il simbolismo politico incarnati dalla Promachos avrebbero raggiunto il culmine soltanto nel momento in cui la quadriga ripristinata, che commemorava il primo vero successo militare della democrazia, avesse trovato posto di fianco ad essa $4^{402}$ A questo punto sarebbe legittimo domandare, se effettivamente la Promachos avesse dovuto acquistare tale pienezza di significato dalla presenza della quadriga, perché quest'ultima non avrebbe potuto essere rifatta in quel luogo direttamente!

Maggiore peso devono invece avere considerazioni di tipo religioso, quali quelle sviluppate da Furtwängler e successivamente da Weber: ${ }^{403}$ una dedica ad Atena al di fuori del temenos consacrato alla divinità non è possibile; come si sa, i doni votivi erano posti, anzi accumulati in competizione fra di loro ("nur an einem gemeinsamen Ort ihre kompetitive Wirkung entfalten konnten”!), ${ }^{404}$ all'interno dei san-

400 V. par. 3.2.17.

401 KeEsling, The Votive Statues, 12; Pedley, Sanctuaries and the Sacred, 18990; ma v. in questo senso già MiLler, A History of the Akropolis, 503.

402 HuRwit, The Athenian Acropolis, 153.

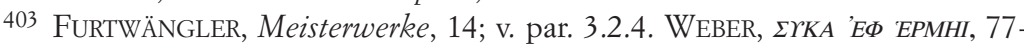
108; v. par. 3.2.11.

404 T. HÖLSCHER, Öffentliche Räume in frühen griechischen Städten, «Ktéma», 23 (1998), 159-170: 163-64; ID., Öffentliche Räume in frühen griechischen Städten, Heidelberg 1998 (Schriften der Philosophisch-historischen Klasse der Heidelberger Akademie der Wissenschaften, 7), 47-48. 
tuari, ${ }^{405}$ e proprio questo era l'Acropoli, sempre meno fortezza e sempre più centro religioso già a partire dal VII secolo. ${ }^{406}$ Del resto è sufficiente confrontare il corpus delle iscrizioni attiche sino alla fine del V secolo per accorgersi che una dedica ateniese ad Atena in Atene in questo periodo ${ }^{407}$ non può che essere all'interno dell'Acropoli: ${ }^{408}$ le dediche ritrovate al di fuori dell'Acropoli sono tutte in favore di altre divinità. ${ }^{409}$

Weber aveva per di più osservato che la pietas religiosa doveva spingere gli Ateniesi a rinnovare la dedica non solo all'interno dell'Acropoli, ma anche e soprattutto nella medesima posizione della dedica originale (pure le uguali dimensioni delle basi vanno in questo senso): è nota la cura dei Greci nella conservazione dei santuari. ${ }^{410}$

E' poi altamente probabile la vicinanza della quadriga ai ceppi cui si fa riferimento in entrambe le versioni dell'epigramma dedicatorio. Si è visto come la localizzazione maggiormente accettata per i ceppi sia il muro di sostegno del Tempio arcaico di Atena. ${ }^{411}$

405 V. p. es. F. GRAF, Gli dei greci e i loro santuari, in I Greci. Storia cultura arte e società, II.1, Torino 1996, 343-80: 353; cfr. BERGQUIST, The Archaic Greek Temenos, 100-06: solo gli oikoi e altre costruzioni minori si trovavano al di fuori dei recinti sacri (comunque ai lati di essi), mentre le offerte votive erano all'interno, "preferably placed at roads and boundaries, at the façades and the flank walls of temples and altars and of non-essential buildings, and at the north and south sides of the sacrificial areas".

406 PEDLey, Sanctuaries, 186-90; di demilitarizzazione dell'Acropoli e della conseguente trasformazione da fortezza in santuario ha parlato E. VANDERPOOL, The date of the pre-Persian City-Wall of Athens, in $\Phi O P O \Sigma$ : Tribute to Benjamin Dean Meritt, ed. by D.W. BradeEn - M.F. MCGregor, Locust Valley, NY 1974, 156-60: 159-60; sul nuovo "approccio" (che può essere inteso qui ad un tempo sia come modalità di accedere all'Acropoli sia come modo di considerarla) che ne deriva v. MYLONAS SHEAR, The Western Approach, 105-07. Per M. C. Hellmann, poi, il reimpiego dei rocchi di colonna del Tempio arcaico di Atena o del cantiere del Pre-Partenone per le mura segnalerebbe ancor più il carattere di recinto sacro dell'Acropoli (M.-C. HeLlmanN, L'architecture grecque, II, Paris 2006, 176).

407 Le dediche e il culto ad Atena sull'Acropoli rimangono dominanti sino al IV secolo; il declino si estende dal IV al I secolo, cf. D. GEAGAN, Who was Athena?, in Religion in the Ancient World. New Themes and Approaches, ed. by M. DiLlon, Amsterdam 1996, 145-64: 147-54.

408 IG, I. $2^{3}$, ed. LEWIS - JEFFERY adiuv. ERXLEBEN, 489-651.

$409 I G, I .2^{3}, 651-55$.

410 Cfr. W. BuRKERT, La religione greca di epoca arcaica e classica, Milano $2003^{2}$ (trad. it. riv. di Griechische Religion der archaischen und klassischen Epochen, StuttgartBerlin-Köln 1977), 194.

411 V. par. 3.2.12. 
Proprio a quest'area porta l'analisi che fa Preißhofen di alcune testimonianze, che, insieme con il passo erodoteo in questione, permettono di individuare il Tempio arcaico di Atena come centro di culto, ove dedicare i trionfi della città alla divinità protettrice: ricordiamo, oltre a ceppi e quadriga di cui si discute, l'esplicita indicazione di uno scolio alla Lysistrata di Aristofane (la stele che registrava i nomi degli Ateniesi giustiziati proprio perché collaborarono con Cleomene, collocata "presso l'antico tempio") e di un passo della Vita Cimonis di Plutarco (gli scudi sottratti ai nemici appesi intorno al medesimo tempio). ${ }^{412}$ Anche Curtius aveva evidenziato gli strettissimi legami fra la vita della comunità cittadina in pace e in guerra e questo tempio in particolare, pur senza trarne le dovute conclusioni topografiche in relazione alla nostra quadriga. ${ }^{413}$ Alle dediche menzionate si potrebbe forse aggiungere anche la celebre consacrazione ad Atena, da parte di Alessandro Magno, di trecento panoplie persiane, inviate ad Atene come dedica sull'Acropoli dopo la battaglia al fiume Granico (334 a.C.) ? ${ }^{414}$ Non sarà impossibile che siano state esposte come trofeo nella medesima area, quella anticamente del Tempio arcaico di Atena, impregnata di ricordi tangibili delle vittorie sui nemici e dominata dal monumento più imponente ad esse relativo, la Promachos? Come nota A.B. Bosworth, proprio quel tempio era stata la vittima principale della distruzione persiana, dunque necessariamente Alessandro vi invia le primizie della vendetta; ${ }^{415}$ anche se va detto che in questo caso solitamente si ipotizza che una parte degli scudi fosse appesa all'epistilio orientale del Partenone, ove sono particolarmente visibili dei fori che ne avrebbero permesso il fissaggio, ${ }^{416}$ una collocazione unica vicino ai

412 Preißhofen, Zur Topographie, 82-83; le fonti analizzate sono Schol. in Aristoph. Lys. 273 e Plut. Cim. 5, 3 rispettivamente; v. par. 3.2.18.

413 CurTIus, Die Stadtgeschichte, 154: "Das alte Heiligthum der Burggöttin behielt immer einen gewissen häuslichen Charakter, insofern hier solche Weihegaben verinigt waren, die mit dem bürgerlichen Leben der Gemeinde in Krieg und Frieden am nächsten zusammenhingen".

414 Arr. An. I 16, 7; cfr. Plut. Alex. 16, 17-18 (che parla solo di scudi).

415 A.B. BosworTH, A Historical Commentary on Arrian's History of Alexander, I, Oxford 1980, 127.

416 Cfr. ad es. JudeIch, Topographie von Athen, München $1931^{2}$, 88; H.R. Goette, Athens, Attica and the Megarid. An Archaeological Guide, London-New York 2001 (trad. ingl. riv. di Athen, Attika, Megaris. Reiseführer zu den Kunstschätzen und Kulturdenkmälern im Zentrum Griechenlands, Köln-Weimar-Wien 1993), 39; W. WILL, Athen und Alexander. Untersuchungen zur Geschichte der Stadt von 338 bis 322 v. Chr., 
resti del Tempio arcaico, se si considera pure il noto legame di Alessandro con Atena Polias, sarebbe stata anche più simbolica. ${ }^{417}$

Ad ogni modo, a prescindere da quest'ultima ipotesi, ciò che importa sottolineare è la continuità di culto nell'area almeno sino all'età classica, indipendentemente dalla più o meno parziale conservazione $o$ ricostruzione del Tempio arcaico di Atena ${ }^{418}$ e indipendentemente dal fatto che il suo frontone possa avere in parte condiviso, almeno con la dedica della quadriga originale, un motivo di ispirazione: il carro da

München 1983 (Münchener Beiträge zur Papyrusforschung und antiken Rechtsgeschichte, 77), 57 n. 58 (che aggiunge l'epistilio occidentale); HuRWIT, The Athenian Acropolis, 254 ipotizza che il resto dell'armatura o almeno gli scudi rimanenti siano stati esposti all'interno del Partenone.

417 Secondo Holtzmann, L'acropole, 214 niente assicura che le tracce degli scudi sulle architravi del Partenone siano da attribuire alle offerte di Alessandro: la pratica di offrire scudi per commemorare una vittoria era molto diffusa. M. KORRES, The Parthenon from Antiquity to the 19th Century, in The Parthenon and Its Impact in Modern Times, ed. by P. TOURNIKIOTIS, Athina 1994, 137-61: 138 ha comunque distinto cronologicamente due serie di dediche di scudi applicati al Partenone, la prima della fine del IV secolo (compatibile quindi con la dedica di Alessandro), la seconda risalente al III secolo. Per le prove del legame di Alessandro con Atena Polias v. G. SQuillace, Alessandro e l'offerta ad Atena di trecento panoplie, in «Miscellanea di studi storici», 9 (1992-1994), 9-20; ID.,

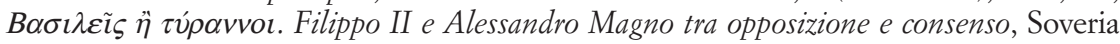
Mannelli 2004: 154-57.

418 I termini della questione furono posti da DÖRPFELD, Der alte Athena-Tempel auf der Akropolis zu Athen «MDAI(A)», 12 (1887): 25-61; ID., Der alte Athena-Tempel auf der Akropolis zu Athen, «MDAI(A)», 12 (1887), 190-211; ID., Der alte Athena-Tempel auf der Akropolis zu Athen, «MDAI(A)», 15 (1890): 420-439, sostenitore della teoria della ricostruzione; contra, tra gli altri, J.G. FRAZER, The Pre-Persian Temple on the Acropolis, «JHS», 13 (1892/93), 153-87; bibliografia completa in Travlos, Pictorial Dictionary, 143. Il consenso è attualmente per la teoria che ne prevede il funzionamento nella sua forma ridotta alla parte occidentale fino all'incendio del 406 a.C. (Pausania, I, a c. di BESCHI Musti, 362; per l'incendio v. U. BuCHERT, Denkmalpflege im Antiken Griechenland. Massnabmen zur Bewährung historischer Bausubstanz, Frankfurt am Main 2000, 216-17, con discussione delle fonti, specialmente Xen., Hell., I 6, 1; IG II ${ }^{2}$ 1654; Dem. XXIV 136) o anche sino a metà del IV secolo (Travlos, Pictorial Dictionary, 143). Molto scettico in materia rimane HuRWIT, The Athenian Acropolis, 143-44. Per una conservazione sino all'avanzata età romana invece U. BUCHERT, Denkmalpflege, 203-25 (il giuramento di Platea sarebbe stato invocato a posteriori per giustificare restauri solo parziali) e G. FERRARI, The ancient temple on the Acropolis at Athens, «AJA», 106 (2002): 11-35 (il giuramento di Platea sarebbe rispettato proprio dalla ricostruzione parziale, che, giustapposta alle rovine, farebbe assumere al tempio lo status di memoriale, una sorta di Gedächtniskirche ante litteram); contra J. PAKKANEN, The Erechtheion Construction Work Inventory (IG $1^{3}$ 474) and the Dörpfeld Temple, «AJA», 110 (2006), 275-81. 
guerra della divinità, richiamato a questo proposito da Rausch, ricorreva certo nell'iconografia in genere, ${ }^{419} \mathrm{ma}$ si è pensato potesse anche essere rappresentato nell'ambito di una Gigantomachia, ${ }^{420}$ sul vicino frontone occidentale del Tempio arcaico di Atena ${ }^{421}$ Ormai bisogna sicuramente tenere conto di diverse ipotesi di ricostruzione di quel frontone, ove la presenza della quadriga è stata pure revocata in dubbio; peraltro Despinis ne garantirebbe poi comunque l'esposizione, da parte del giovane Stato democratico committente del progetto incompiuto dei nuovi frontoni, sul terreno immediatamente sottostante in forma di dedica, a questo punto in tutti i sensi vicinissimo precedente della nostra. ${ }^{422}$ Qualsiasi soluzione si voglia adottare, però, si è visto come la continuità di culto ad Atena nell'area, più in generale, del Tempio arcaico, non possa certo essere negata: ecco perché bisogna pensare che la quadriga ed il suo rifacimento dovessero trovarsi, come i ceppi e in quanto dediche di vittorie cittadine alla divinità poliade, nei pressi di ciò che rimaneva di quel tempio; non lontano, quindi, dalla Promachos.

E' stato obiettato che allora Erodoto ne avrebbe descritto la collo-

419 RAUSCH, Isonomia, 124 con nn. 569-72: particolarmente interessanti le rappresentazioni su tavole di terracotta (l'una dipinta, l'altra a rilievo) riportate da DEMARGNE, Athena, in LIMC, II.1, $\mathrm{n}^{\mathrm{i}}$ 175-76 (= LIMC, II.2, tav. $\left.724 \mathrm{n}^{\mathrm{i}} 175-76\right)$, in quanto dediche private coeve (fine del VI secolo) e provenienti proprio dall'Acropoli di Atene.

420 Livingstone - NisBet, Epigram, 34 n. 38 non escludono addirittura un'identificazione dei vincitori ateniesi con le divinità olimpiche nelle varie GigantoTitanomachie, ove, come nel nostro epigramma, è schiacciata l'bybris dei nemici e que-

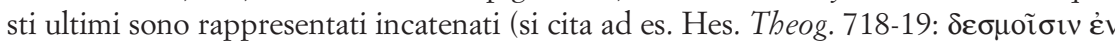

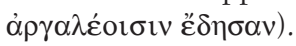

${ }^{421} \mathrm{~V}$. par. 2; Atena è al fianco di una quadriga, che poteva essere occupata da Zeus. Sicurissimo della relazione fra la nostra quadriga ed il frontone occidentale del Tempio arcaico di Atena JüngER, Gespann und Herrschaft, 239 n. 698.

422 Per MARSZAL, An Epiphany, 173-80 la quadriga, con al centro Atena in persona, apparterrebbe al frontone opposto: v. par. 2; di solito al frontone orientale si attribuiscono invece soltanto i due leoni che sbranano un toro, che riprenderebbero nell'iconografia la precedente fase costruttiva (cfr. brevemente H. KNELL, Mythos und Polis. Bildprogramme griechischer Bauskulptur, Darmstadt 1998, 39-40 con fig. 54-55); come abbiamo visto, per SANTI, I cavalli inv. 6454 e 15244 del Museo dell'Acropoli, 1-23, la quadriga non può essere ricondotta ad alcuno dei frontoni; più possibilista K. KISSAS, Archaische Architektur der Athener Akropolis. Dachziegel - Metopen - Geisa Akroterbasen, Wiesbaden 2008 (Archäologische Forschungen 24), 60 con note, comunque contrario all'appartenenza della quadriga al frontone occidentale. L'ipotesi di DESPINIS, Der Torso, 5-7 identifica il torso NM 3045 con la statua di Zeus con Atena 
cazione facendo riferimento alla colossale statua di Atena, invece di parlare dei Propilei; $;{ }^{423}$ ma così facendo si rischia di incorrere nella vecchia pretesa di "insegnare a scrivere" agli autori antichi, e a questa stregua si potrebbe benissimo citare, contro la proposta corrente di localizzare la quadriga dinanzi ai Propilei, le precise parole di Erodoto quando intende veramente indicare che delle statue si trovano davanti a un'entrata: $\alpha \dot{v}$ -


(e non solo per lui) semplicemente l'espressione con valore topografico "a sinistra (a destra) per chi entra" è d'uso comune: probabilmente è la più immediata e naturale a disposizione in questi casi $^{425}$ (il che esclude anche che Erodoto volesse utilizzarla con un valore temporale forzato).

Ebbene, come si spiega l'indicazione erodotea "a sinistra per chi entra nei Propilei dell'Acropoli?" Se si vogliono accettare, tra quelli proposti, i tagli nella roccia interni all'Acropoli scelti da Stevens, ${ }^{426}$ che godono dei maggiori consensi, bisognerebbe pensare a un riorientamento del visitatore appena superati i Propilei, come già sostenuto da Weber. ${ }^{427}$ Ed effettivamente le analisi di G.P. Lavas hanno evidenziato che il visitatore dell'Acropoli arcaica doveva di necessità girarsi verso destra per avere percezione dei templi una volta attraversati gli Antichi Propilei, dato che questi ultimi erano orientati decisamente a Nord-Est; ma anche in età classica la vista spaziava sull'Acropoli soltanto dopo

nascente descritta da Pausania (I 24, 1) a Nord-Ovest del Partenone e ne ricostruisce la genesi, insieme con i cavalli Akr. 6454 e Akr. 15244, in vista di un progetto di sostituzione dei frontoni del tempio arcaico di Atena mai realizzato compiutamente e conclusosi con l'esposizione su basi, proprio come avvenne a Egina, delle uniche sculture terminate (in questo caso dietro il tempio, a Ovest; a Egina le opere "concorrenti" furono esposte a Est: v. A. FuRTWÄngler, Aegina. Das Heiligtum der Aphaia, München 1906: 256-74).

423 MeIGGS - LEWIS, Greek Historical Inscriptions, Oxford 1969, 29 n 15.

${ }^{424}$ Her. II 121, 1 a proposito delle statue dell'Estate e dell'Inverno presso i pro-

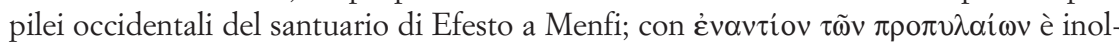
tre localizzato il cortile di Api fatto costruire da Psammetico di fronte ai propilei meridionali (sempre del santuario di Efesto a Menfi: Her. II 153, 1).

425 Medesima espressione in Her. I 51, 1; II 169, 4; IV 34, 2; simile (col semplice ióvचı) in Her. VII 198, 2; si tratta della normalissima costruzione con il dativo di relazione (altri esempi erodotei con altri verbi di movimento: II 155, 1; III 90, 2).

426 Stevens, The Periclean Entrance Court, 492 fig. 42 taglio non numerato immediatamente a Sud del $n^{\circ} 2$ (collocazione della dedica originale); 492 fig. $42 n^{\circ} 2$ (collocazione della copia); v. par. 3.2.16.

427 WEBER, ГrKA 'EФ 'EPMHI, 95-96; 100-02; v. par. 3.2.13. 
essere entrati attraverso i Nuovi Propilei maggiormente in asse con l'Oriente. ${ }^{428} \mathrm{~A}$ prescindere quindi da quale porta entrasse il visitatore (non da quella centrale) ${ }^{429}$ e anche da quali Propilei oltrepassasse (il dibattito è ancora aperto), ${ }^{430}$ il punto di vista doveva formarsi appena imboccata la strada principale dell'Acropoli. Proprio questa strada, peraltro, doveva dividere in due parti l'Acropoli: a sinistra l'area afferente al Tempio arcaico di Atena Polias, a destra l'area del Partenone. ${ }^{431}$ Ed è per questo motivo, poiché ceppi e quadriga appartenevano all'area del Tempio arcaico di Atena, che, una volta entrati e imboccata la strada principale, si poteva dire "a sinistra". Sarebbe però forse possi-

428 G.P. LAVAS, Altgriechisches Temenos: Baukörper und Raumbildung, BaselStuttgart 1974, 32-33.

429 WeIzsäCKer, Pausanias und die Bildwerke, 7; Stevens, The Periclean Entrance Court, 451; è stato proposto che si entrasse sulla destra negli Antichi Propilei (PritchetT, The Liar School, 157) e sulla sinistra nei Propilei mnesiclei (Stevens, The Periclean Entrance Court, 451-55); contra, per un uso costante - in quanto d'origine religiosa - di entrare nei santuari dalla porta destra e uscirne dalla sinistra, ma destra per chi esce, compiendo cioè "et intrantes et exeuntes dexstrorsum iter", BERGK, Poetae Lyrici Graeci, III, 478, citando a sostegno un epigramma trasmesso dall'Antologia Palatina (IX 758), solitamente classificato tra quelli attribuiti a Simonide (ep. 162 BERGK = 163 DIEHL = XXXIII PAGE); per il commento cfr. anche D.L. PAGE, Further Greek Epigrams, Cambridge 1981, 246.

430 R.W. Macan, Herodotus. The 4th, 5th and 6th Books, I, London-New York 1895, 223 osservava come Erodoto parlasse solo qui di $\pi \rho 0 \pi v i \lambda \alpha \alpha$ ateniesi, utilizzando invece per tre volte, nel corso della descrizione dell'assedio dell'acropoli del 489, il ter-

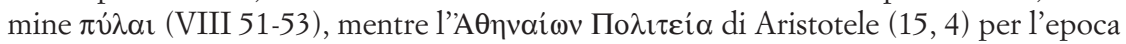

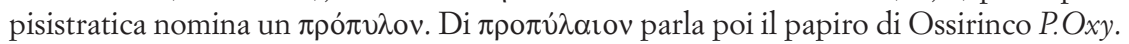
2535, ma proprio commentando l'epigramma per la vittoria ateniese sui Beoti e sui Calcidesi! E' stato più volte notato che la definizione plurale di $\pi \rho \circ \pi v ́ \lambda \alpha \iota \alpha$ o $\pi \rho{ }^{\prime} \pi \nu \lambda \alpha$ ricorre altrove nelle Storie solamente a proposito degli ingressi monumentali di santuari egiziani (v. SCHOLLMEYER, Antike Gespanndenkmäler, 56-57 n. 304 con i passi erodotei), ma secondo HuRwIT, The Athenian Acropolis, 144 nel caso di Atene potrebbe essere applicata indifferentemente ai Nuovi Propilei come agli Antichi, poiché anche questi avevano più soglie. Non si può quindi dedurre per via lessicale che i $\pi \rho \pi_{0} v_{\lambda} \alpha \mathbf{\alpha}$ ateniesi menzionati qui da Erodoto fossero quelli di Mnesicle; tanto più che il commentario papiraceo P.Oxy. 2535, o meglio la sua fonte, parlava, nel medesimo contesto, di $\pi \rho \circ \pi \dot{\lambda} \alpha i o v !$

431 Che le strade servissero a suddividere in parti l'Acropoli è sottolineato da KORRES, The History, 39. Di tre parti parla LAVAS, Altgriechisches Temenos, 31 considerando anche lo spazio intermedio, ma più da un punto di vista ottico che funzionale o religioso. 
bile una spiegazione ancora più semplice, qualora si rinunciasse del tutto ai tagli assegnati da Stevens alla quadriga e si volesse cercare un'altra collocazione, più nettamente a sinistra appena entrati nell'Acropoli (lo suggerisce il $\pi \rho \tilde{\omega} \tau o v$ di Her. V 77, 4), a una limitata distanza dal muro di sostegno del Tempio arcaico di Atena al quale sarebbero stati appesi i ceppi ${ }^{432}$ e magari non così lontano neppure dal luogo di ritrovamento delle iscrizioni, descritto con precisione, nel caso del frammento di VI secolo, da Lolling:433 è vero che non c'è garanzia che il frammento appartenente a quel blocco sia stato sotterrato nel medesimo punto in cui era esposto, ma è un fatto che il luogo descritto da Lolling ${ }^{434}$ si trova effettivamente subito a sinistra per chi entra nell'Acropoli attraverso i Propilei (sia Antichi sia Nuovi: cadrebbe a questo punto anche la necessità di distinguere a quali si riferisse Erodoto). Né si può certo dire che una collocazione di questo tipo possa essere in contrasto con l'indicazione topografica data dal frammento di commentario papiraceo P.Oxy 2535. ${ }^{435}$

Del resto neppure le identificazioni di Stevens sono sicure (sia per le dimensioni, sia - lo si è visto - per essere influenzate da teorie precedenti non inoppugnabili): ${ }^{436}$ il dibattito sull'assegnazione dei vari tagli individuati per via archeologica ai monumenti noti da testimonianze letterarie è ancora in corso, e c'è sempre il rischio di riempire i vuoti delle nostre conoscenze con quanto invece ci è noto, attribuendo questo o quel taglio alla famosa quadriga; inversamente Stevens stesso poteva dedurre in alcuni casi la presenza di strutture non ricordate dalla tradizione, ${ }^{437}$ il che a questo punto potrebbe valere pure per altri tagli in questione, anche considerata la presenza accertata di un gran numero di altre dediche, note e meno note. Non è detto, quindi, che si tratti dei tagli destinati alla quadriga, né che i tagli adatti siano dove si è cercato finora, né forse - che sia possibile individuarli. In mancanza di una prova definitiva è quindi preferibile non escludere alcuna possibilità, ovviamente una

432 V. par. 3.2.12.

433 Lolding in KirchHoff (Lolling), Bemerkungen, 112 (= Lolling in IG, I suppl., ed. KIRCHHOFF, 78 n $^{\circ} 334$ a).

434 L'edificio a Nord-Est dei Propilei, a Est della Cisterna, a pochi passi dal portico orientale dei Propilei. V. n. 22.

435 Sappiamo però che non si può dire molto di più in merito: v. par. 1.

436 V. par. 3.2.16.

437 STEVEns, The Periclean Entrance Court, 492 fig. 42 n $\mathrm{n}^{\mathrm{i}}$ 10, 11, 12, 13; 509-10. 
volta ristretto il campo alle vicinanze del Tempio arcaico di Atena. Un'interpretazione meno rigida eppure aderente al testo di Erodoto e di Pausania permetterebbe così di evitare eccessive contraddizioni: se infatti da un lato notava scherzosamente R.W. Macan come "Herodotus conceived himself to be writing history not compiling a guide-book", ${ }^{438} \mathrm{dal}-$ l'altro è proprio Pausania a sembrare in questo caso molto meno interessato a dare informazioni sulla posizione della quadriga, non utilizzando una voluta indicazione topografica come quella di Erodoto. Non dovremo quindi essere costretti a postulare la vicinanza anche della Promachos alla nostra quadriga: non è quanto scrive Pausania, che accomuna i due monumenti perché dediche ad Atena della decima di un bottino di guerra, il che non esclude affatto la vicinanza, ma neppure la richiede espressamente; si può cioè immaginare che siano entrambi visibili in un solo colpo d'occhio, ma non necessariamente una a fianco dell'altra. E' già stato osservato, infatti, che in genere Pausania segnala espressamente quando non segue un ordine topografico (cfr. ad es. Paus. V 14, 4; 10); ma è proprio durante e a proposito della descrizione dell'Acropoli che possiamo trovare un passo a mio giudizio rivelatore del suo modo di procedere, laddove il Periegeta, dopo aver illustrato il Partenone e, più oltre, la statua di Apollo Parnopio, ${ }^{440}$ nomina una prima volta la statua di Pericle insieme con quella del padre Santippo, in un raggruppamento evidentemente tematico, e subito si preoccupa di precisare: $\dot{\alpha} \lambda \lambda^{\prime} \dot{o} \mu \grave{\varepsilon} v$

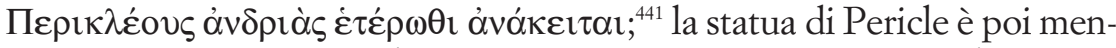
zionata insieme con l'Atena Lemnia appena prima di uscire

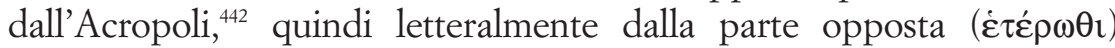
rispetto alla statua del padre. Dunque Pausania può in buona sostanza essere seguito nel suo itinerario, dall'ingresso nell'Acropoli al santuario di Artemide Brauronia al Partenone all'Eretteo alla casa delle Arrefore all'uscita: ${ }^{443}$ è giusto prima di uscire che descrive la Promachos e accenna alla quadriga; segue la descrizione delle mura e la graduale discesa

438 R.W. MaCAN, Herodotus, I, 223.

439 V. par. 3.1.11.

440 Paus. I 24, 5-8.

441 Paus. I 25, 1.

442 Paus. I 28, 2. Dovevano trovarsi presso l'angolo Nord-Est della fronte orientale dei Propilei (Pausania. Guida della Grecia, I, a c. di BesCHI - Musti, 368; JudeICH, Topographie von Athen, München 19312, 232 n. 4).

443 Paus. I 22, 4-28, 4. 
dall'Acropoli. Non si può perciò negare che Pausania vedesse la quadriga all'interno dell'Acropoli, e probabilmente, se l'ordine topografico era mantenuto, in un luogo compreso fra l'Eretteo, i resti del Tempio di Atena Polias e la Casa delle Arrefore: una localizzazione effettivamente "a sinistra per chi entra" e compatibile con il luogo di ritrovamento.

Proprio nella stessa zona, presso il Tempio di Atena Polias, Pausania ricorda pure la presenza di una statua dello stratego Tolmide; ${ }^{44}$ potrebbe non trattarsi di un caso, se è corretta l'ipotesi che ho già avuto modo di esporre, ${ }^{445}$ secondo la quale l'occasione per il rifacimento della quadriga per la vittoria sui Beoti e sui Calcidesi del 506 a.C. sarebbe stata, oltre al grandissimo successo di Mironide a Enofita sui Beoti (457 a.C.) ${ }^{446}$ anche (e un po' propagandisticamente) l'affermazione su altri Calcidesi, quelli d'Etolia, da parte di Tolmide nel corso del suo periplo del Peloponneso di poco successivo (456/5 a.C.): $:^{447}$ se è vero, non ci poteva essere luogo migliore per dedicare (a prescindere da chi lo abbia fatto) una effigie di Tolmide, presso un monumento - la quadriga per la vittoria sui Beoti e i Calcidesi - al quale, anche in seguito al di lui contributo militare (certo in sé non paragonabile a quello del collega Mironide, ma sono note le ambizioni di Tolmide in merito), ${ }^{448}$ era stato dato ulteriore significato. Sebbene non si pretenda qui di attribuire un valore di prova a questa collocazio-

444 Paus. I 27, 5 . Il passo sarà analizzato in un mio prossimo articolo.

445 BeRTI, La dedica degli Ateniesi, 22-24; l'intuizione risale a Ph.-E. Legrand (Hérodote. Histoires, V, établi et traduit par PH.-E. Legrand, Paris 1946, 36 n. 1).

446 Thuc. I 108, 3; per la cronologia, il contesto storico e una prima discussione delle fonti parallele v. per es. A.W. Gomme, A Historical Commentary on Thucydides, I, Oxford 1945, 315-20 e S. Hornblower, A Commentary on Thucydides, I, Oxford 1991, 171-73; in generale sulla cronologia (tucididea o meno) della Pentecontaetia v. soprattutto E. Badian, From Platea to Potidaea. Studies in the History and Historiography of the Pentecontaetia, Baltimore-London 1993, 73-107; 201-15 e recentemente P.J. RHODES, Thucydidean Chronology, «AAntHung», 49 (2009), 353-58.

447 Thuc. I 108, 5. Semplicemente $\mu \varepsilon \tau \dot{\alpha} \tau \alpha \tilde{v} \tau \alpha$ per Tucidide; la data è ricavata da Diod. XI 84, 1-2, che concorda con Schol. in Aeschin. II 75 in relazione al periplo del Peloponneso, ricordato anche da Paus. I 27, 5. La datazione diodorea, già approvata da Wilamowitz (U. von Wilamowitz-MoellendorfF, Aristoteles und Athen, II, Berlin 1893, 294), è ormai condivisa dalla critica: v. BADIAN, From Platea, 214 n. 53; V. PARKER, The Chronology of the Pentecontaetia from 465 to 456, «Athenaeum», 81 (1993), 129-47: 142-44; Diodorus Siculus, Books 11-12.37.1. Greek History 480-431 B.C. - The Alternative Version, transl., with intr. and comm., by P. GREEN, Austin 2006, 162 n. 338.

448 Diod. XI 84, 2 (all'origine del periplo del Peloponneso il desiderio da parte di 
ne, ${ }^{449}$ bensì al massimo di conferma del quadro tracciato sinora di una dedica della quadriga nei pressi del Tempio arcaico di Atena, è innegabile che per l'osservatore antico era una domanda significativa, in quale contesto fosse posto un ritratto: ${ }^{450} \mathrm{~T}$. Hölscher ha raccolto molti esempi in questo senso, classici ed ellenistici (in parte sino all'età romana). ${ }^{451}$

Molto (più) rilevante, anzi decisiva apparirà allora la collocazione, sempre presso l'archaios naos e quindi non lontano dalla nostra quadriga, della stele degli Ateniesi che avevano collaborato con Cleomene, la quale, insieme con altre testimonianze, era servita a Preißhofen per sottolineare la continuità di culto in un'area vicina al Tempio arcaico di Atena, consacrata alle dediche della città trionfante sui propri nemici: ${ }^{452}$ ma il suo significato è molto maggiore in relazione alla quadriga. Infatti noi sappiamo che entrambe, quadriga e stele che potremmo chiamare "dei collaborazionisti”, ricordano gli stessi avvenimenti: con tutta probabilità furono rinnovate nella stessa occasione. L'attacco alla democrazia ateniese alla fine del VI secolo è portato avanti su più fronti: ${ }^{453}$ all'interno dai partigiani di Isagora, all'esterno da Cleomene spartano con gli alleati peloponnesiaci e la partecipazione dei Beoti e dei Calcidesi; la collaborazione degli ateniesi filo-oligarchici con il nemico esterno è poi severamente punita: sappiamo dal già citato scolio alla Lysistrata che gli Ateniesi condannarono a morte quanti avevano occupato Eleusi insieme con Cleomene, distruggendone le case e confiscandone i beni, ma soprattutto apprendiamo che i nomi di costoro vennero iscritti su di una stele di bronzo posta sull'acropoli appunto presso l'archaios naos. ${ }^{454}$ Se quindi la quadriga celebrava la scon-

Tolmide di compiere un'azione gloriosa degna di Mironide); cfr. successivamente Plut. Per. 18, 2-3 (ambizioni beotiche di Tolmide che culmineranno nella sconfitta di Coronea).

449 Non lontano dalla quadriga si doveva trovare, ad es., pure la statua di Pericle (Paus. I 28, 2; v. n. 441), che però è normalmente associata all'Atena Lemnia (ibidem), cfr. T. HÖLSCHER, Die Aufstellung des Perikles-Bildnisses und ibre Bedeutung, «WJA», N.F. 1 (1975), 187-99: 192; una sintesi equilibrata sulla "politicità" di questo ritratto in R. DI CESARE, Il significato delle korai e altre note sulla scultura votiva dell'Acropoli di Atene, «ASAA», 81 s. 3,3 (2) (2003), 649-64: 660-61.

450 HÖlsCHER, Die Aufstellung, 188.

451 Hölscher, Die Aufstellung, 187-99; ID., Die Nike der Messenier und Naupaktier in Olympia, «DDAI», 89 (1974), 70-111, con schema a p. 79.

452 PREIßHOFEN, Zur Topographie, 82-83; v. par. 3.2.18.

453 Her. V 70-78; Arist. Ath. 20, 2-3; Aristoph. Lys. 273-80.

454 Schol. in Aristoph. Lys. 273. Testo: Scholia in Aristophanis Lysistratam ed. J. HANGARD, Groningen 1996, 16-17. 
fitta dei nemici esterni - gli unici rimasti, i Beoti e i Calcidesi - la stele si ergeva a monito contro i nemici interni. Ma anche quando il monumento fu rifatto, con ogni probabilità in occasione della sconfitta dei Beoti a Enofita $^{455}$ (anche in quel caso lasciati soli dagli Spartani che rientravano ormai in patria) ${ }^{456}$ un monito agli oppositori interni poteva essere d'attualità. C. Bearzot ha difatti raccolto una serie di indizi e notizie nelle fonti antiche, che segnalavano la non troppo remota possibilità in quegli anni di un complotto antidemocratico, e ha posto in evidenza i legami con l'oligarchica Beozia: ${ }^{457}$ l'esplicita testimonianza di Tucidide relativa ad alcuni Ateniesi che speravano di abbattere la democrazia rivolgendosi agli Spartani accampati a Tanagra in Beozia poco prima della battaglia, ${ }^{458}$ contemporaneamente ai sospetti nei confronti dei cimoniani che poi però si riscattarono in battaglia, ${ }^{459}$ mostrano un clima che, alimentato dall'uccisione di Efialte pochi anni prima riconducibile proprio alla "collaborazione attiva tra le forze antidemocratiche ateniesi e quelle beotiche" ${ }^{460}$ poteva certamente giustificare il ripristino della stele che ricordava la punizione dei traditori alla fine del VI secolo: ora come allora, infatti, la vittoria sui Beoti (457 a Enofita) poneva fine d'un colpo alle trame dei nemici di Atene, interni ed esterni.

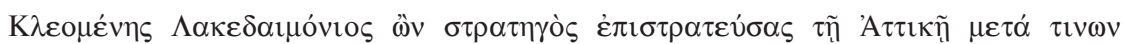

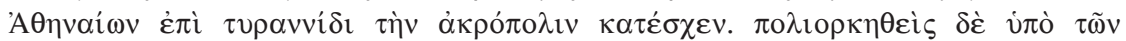

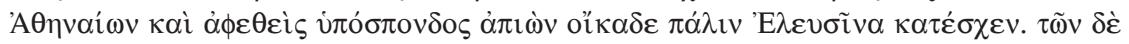

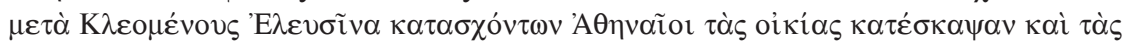

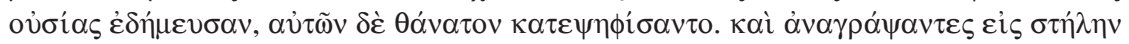

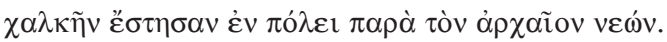

Cleomene spartano quando fu generale fece una spedizione contro l'Attica con alcuni degli Ateniesi e occupò l'Acropoli avendo come obiettivo la tirannide. Assediato però dagli Ateniesi, fu lasciato andare in seguito ad accordi, ma tornando in patria occupò nuovamente Eleusi. Gli Ateniesi distrussero le case e confiscarono i beni di quelli che avevano occupato Eleusi con Cleomene e li condannarono a morte. E li iscrissero su una stele di bronzo che posero sull'Acropoli presso il tempio antico.

455 Cfr. BerTi, La dedica degli Ateniesi, 19-40.

456 Thuc. I 108, 2.

457 C. BEARZOT, La costituzione beotica nella propaganda degli oligarchici ateniesi del 411, in La Béotie Antique, Lyon-Saint-Étienne, 16-20 mai 1983, Paris 1985, 21926: $223-24$ con fonti.

458 Thuc. I 107, 4-6.

459 Plut. Cim. 17, 4-6; Per. 10, 1-2.

460 Bearzot, La costituzione, 223, combinando Arist. Ath. 25, 4 e Plut. Per. 10, 8. 
La stele del VI secolo, necessariamente andata incontro alle distruzioni persiane dell'Acropoli ${ }^{461}$ doveva essere stata ripristinata: le notizie del nostro scolio derivano infatti da una fonte antica, ma di certo successiva (Wilamowitz suggeriva Cratero, ${ }^{462}$ Jacoby, pur non escludendolo, pensava anche ad un attidografo), ${ }^{463}$ che potrebbe ben aver letto l'iscri-

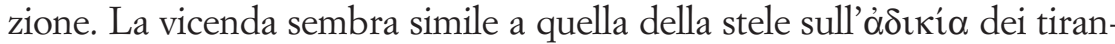
ni, nota per l'uso documentario che ne fa Tucidide (VI 55 1-2), ${ }^{464}$ che rivela un'autopsia dell'iscrizione collocata sull'Acropoli ${ }^{455}$ e ne fa quindi presupporre un analogo ripristino dopo le Guerre Persiane. ${ }^{466}$

E infatti è possibile individuare un intero gruppo di "stele di traditori" innalzate e/o rinnovate sull'Acropoli nel corso dell'esistenza democratica di Atene ${ }^{467}$ la loro concentrazione, in un gioco di rimandi simbolici fra le une e le altre, appunto presso il Tempio arcaico di Atena è stata evidenziata per primo da Wilamowitz ${ }^{468}$ e confermata da Judeich. ${ }^{469}$

461 Her. VIII 53, 2; IX 13, 2.

462 Wilamowitz-MoellendorfF, Aus Kydathen, Berlin 1880, 71; ID., Aristophanes. Lysistrate, Berlin 1927 (= 19582), 139-40; sull'identità e l'epoca in cui potrebbe essere vissuto Cratero v. Cratero il Macedone. Testimonianze e frammenti, a c. di D. ERDAS, Tivoli 2002, 1-46.

463 JACOBY, Die Fragmente der griechischen Historiker, IIIb (Noten), Leiden $1955,66-67$ n. 50.

464 Cf. C. ZızZA, Tucidide e il tirannicidio: il buon uso del materiale epigrafico, «AFLS», 20 (1999), 1-22: 7-12, con bibliografia degli studi precedenti.

465 Così ZizZA, Tucidide e il tirannicidio, 9-11 e C. BEARZOT, L'uso dei documenti in Tucidide, in L'uso dei documenti nella storiografia antica, a c. di A.M. BIRASCHI - P. DESIDERI - S. RODA - G. ZECCHINI, Napoli 2003, 265-314: 294-95.

466 G. Busolt, Griechische Geschichte, II, Gotha 1895, 398 n. 2 (che richiama la sorte delle statue dei tirannicidi); JuDEICH, Topographie von Athen, München $1931^{2}$, 235-36 n. 2.

467 JudeICH, Topographie von Athen, München 19312, 235-36 n. 2.

468 WilamowitZ-MOELLENDORFF, Aus Kydathen, 70-71.

469 JUDEICH, Topographie von Athen, München 19312, 235-36 n. 2, che sottolinea però, seguendo BusOLT, Griechische Geschichte, II, 398 n. 2, come l'ipotesi successiva di WiLAmowitz-MoelLEndorfF, Aristoteles und Athen, I, 114-15, per cui la stelecatalogo dei traditori avrebbe in realtà contenuto non solo i nomi, ma pure tutti i decreti di condanna, non fosse sostenuta dalle fonti, che parlano sempre di singole stele; l'ipotesi, spiegava inoltre Busolt, costringeva il Wilamowitz a postdatare senza motivo (era infatti possibilissimo un rinnovo) l'incisione dei decreti di condanna del VI secolo a dopo le Guerre Persiane, visto che la stele-catalogo risultava soltanto dalla fusione 
Dunque la quadriga, sia l'originale sia il frutto del rifacimento, si collocava concettualmente - ma con tutta probabilità anche fisicamente - all'intersezione di due insiemi simbolici: da un lato quello delle dediche per la vittoria, Promachos compresa (alla quadriga si poteva eventualmente e idealmente accostare, con allusione alla vittoria "calcidese", la statua di Tolmide) e dall'altro i monumenti di biasimo per i traditori, le cui trame furono vanificate in entrambe le occasioni, nel 506 come nel 457 ; in nessun modo, quindi, sarebbe stato lecito distruggere il contesto, a questo punto non solo religioso ma anche politico, formato dalla quadriga, dai ceppi e dalla stele dei traditori ateniesi della fine del VI secolo, con lo spostamento del principale dei suoi protagonisti.

\footnotetext{
della statua dell'ostracizzato Ipparco (Lycurg., 117-18); rimane valido invece il ragionamento che porta al raggruppamento delle stele nel medesimo luogo: la presenza di una stele-catalogo è testimoniata da Licurgo; ne segue la vicinanza ad essa delle altre stele con i corrispondenti decreti di condanna, collocate dalle nostre fonti sull'Acropoli in genere, ma anche più precisamente vicino alla Promachos (Dem. XIX 272, stele di Artmio di Zeleia) e nel nostro caso (Schol. in Aristoph. Lys. 273) presso il Tempio arcaico di Atena. Tutte dunque dovevano essere poste fra la Promachos ed il Tempio arcaico di Atena.
} 


\section{ADDENDUM}

Mentre questa memoria era in corso di stampa sono venuto a conoscenza dell'ulteriore importante contributo di M.C. Monaco alla topografia dell'Acropoli. Oltre a partecipare attivamente al volume già più volte menzionato "Topografia di Atene" (Atene-Paestum 2010), a cura di E. Greco, la studiosa ha infatti recentemente pubblicato anche un articolo (M.C. MONACO, Sull'Acropoli, all'ombra della Promachos, «ASAA», 87, s. III, 9, I (2009-2010), 275-311, giunto in Università Cattolica nel corso del 2011) in cui approfondisce il valore simbolico dell'area circostante l'Atena Promachos, ricostruendone pure la topografia monumentale. L'analisi archeologica, epigrafica e letteraria conferma l'importanza dell'area: ulteriori trofei ed anathemata particolarmente correlati alle Guerre Persiane sono dalla Monaco raggruppati intorno alla Promachos, punto di riferimento fisico e simbolico. La studiosa ha altresì avuto il coraggio, come da me - si parva licet componere magnis - auspicato, di mettere in dubbio, a causa della forma e delle dimensioni, gli stessi tagli nella roccia ritenuti da Stevens "adatti" alla quadriga, vicino alla Promachos, e di proporre una collocazione decisamente "a sinistra per chi entra" per il tanto discusso monumento, senza bisogno di ipotizzarne la traslazione in qualche fase della sua esistenza (tra l'altro anche la Monaco non attribuisce valore di prova all'inversione della coppia di distici dell'epigramma dedicatorio). Sono quindi rassicurato dal fatto di concordare, in alcune mie considerazioni, con un'archeologa esperta. Non sarei così drastico, invece, nel dedurre un totale distacco, fisico e simbolico, della quadriga dall'area della Promachos: proprio l'elevato tasso simbolico dell'agalma non fa escludere una sua influenza nell'intera area da me evidenziata - coincidente con il luogo di ritrovamento dell'iscrizione originale e in parte sovrapponentisi a quella proposta dalla studiosa per la quadriga un'area a sinistra dell'ingresso delimitata e dominata dai Propilei, dalla Promachos e dal Tempio arcaico di Atena, e consacrata alla celebrazione delle vittorie sui nemici non solo esterni, ma anche e soprattutto interni. 